\title{
IntechOpen
}

\section{Practical Applications of Electrocardiogram}

Edited by Umashankar Lakshmanadoss

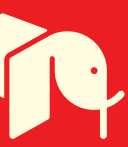





\section{Practical Applications of Electrocardiogram}

Edited by Umashankar Lakshmanadoss 

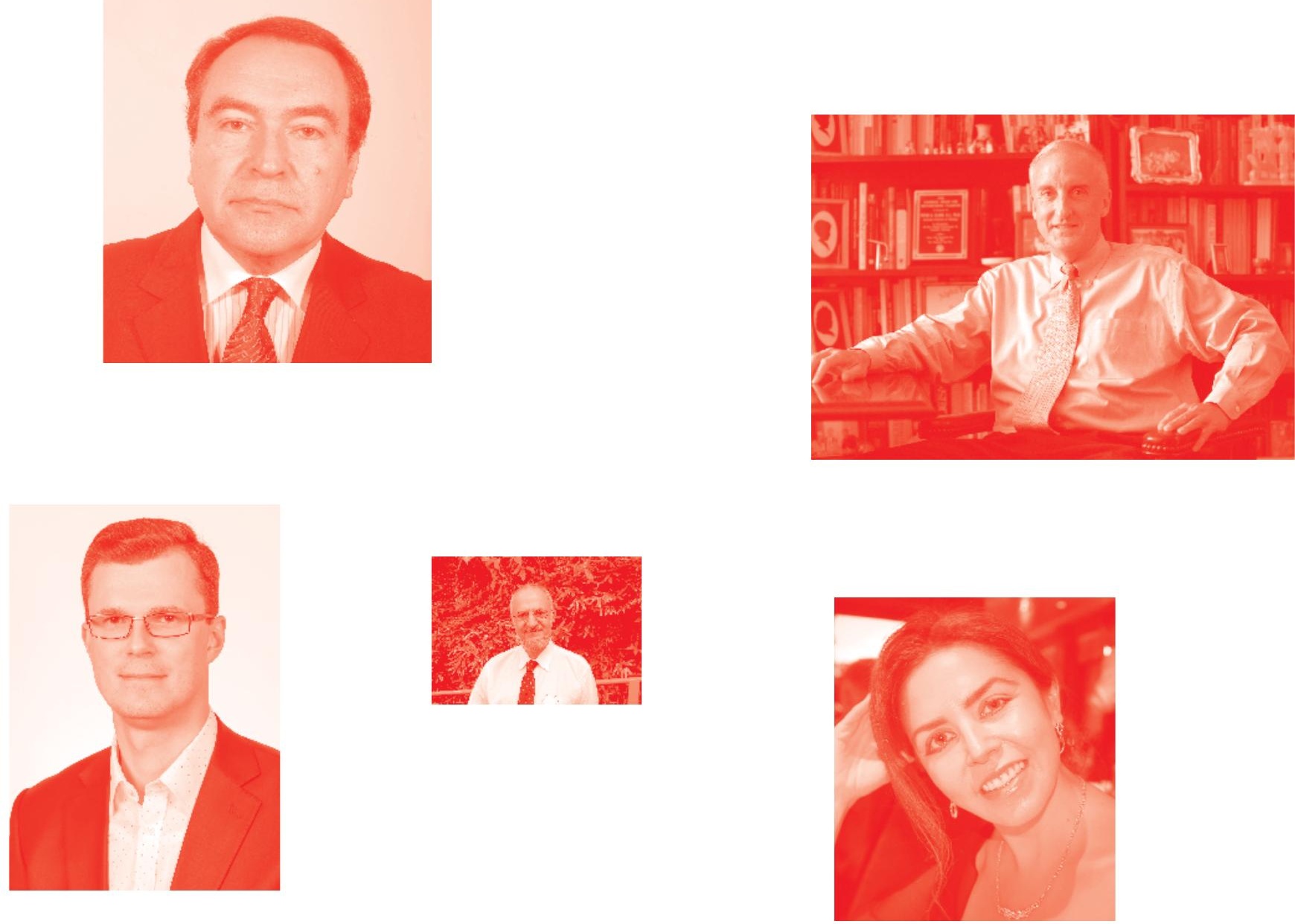

Supporting open minds since 2005
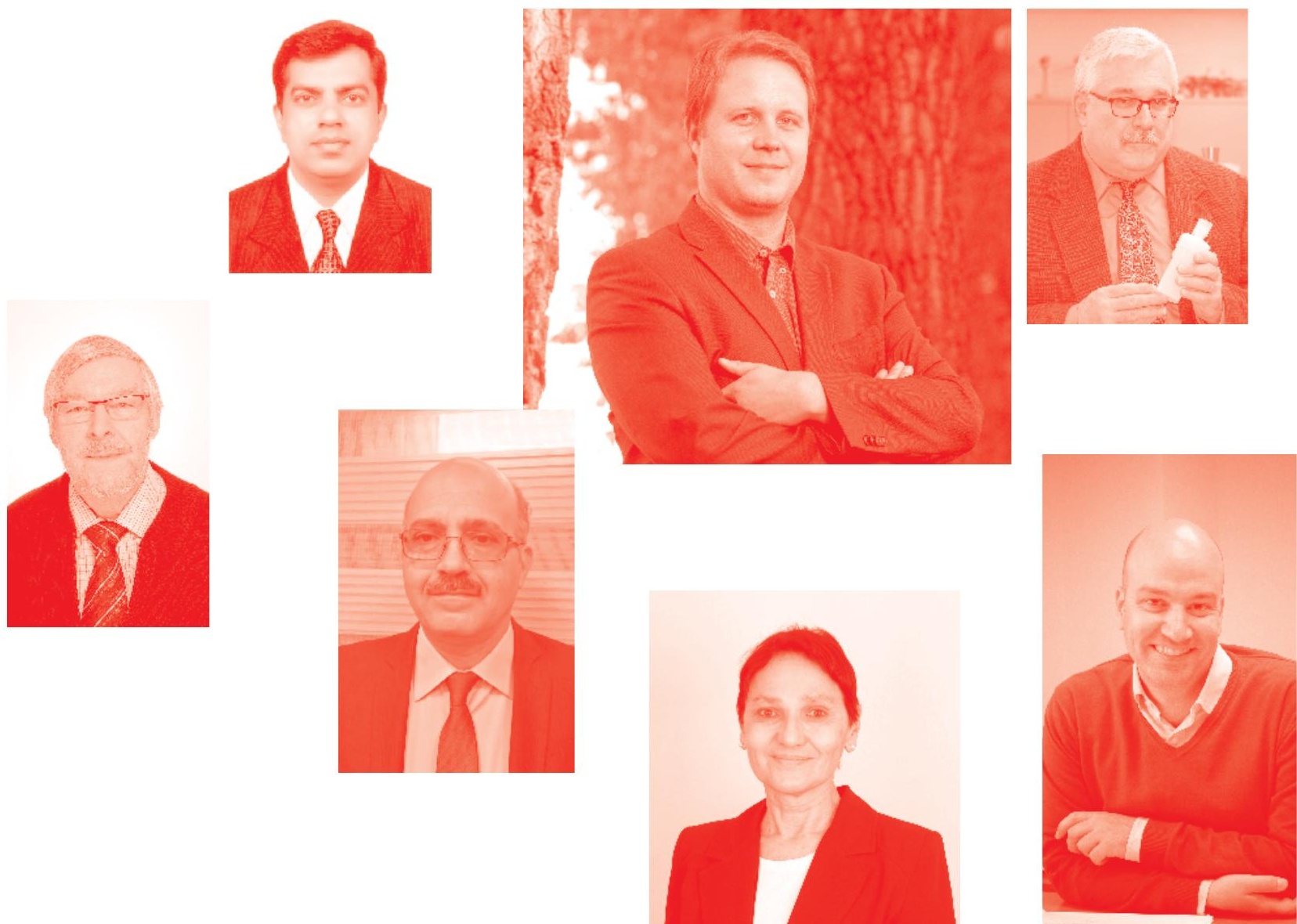
Practical Applications of Electrocardiogram

http : //dx. doi.org/10.5772/intechopen. 77737

Edited by Umashankar Lakshmanadoss

\section{Contributors}

Nevra Alkanli, Arzu Ay, Jeffrey Zhi Jie Zheng, Zhihui Hou, Lihua Leng, Jing Zhang, Xin Gao, Azzedine Dliou, Samir Elouaham, Rachid Latif, Mostafa Laaboubi, Hakan Altay

(๑) The Editor(s) and the Author(s) 2020

The rights of the editor(s) and the author(s) have been asserted in accordance with the Copyright, Designs and Patents Act 1988. All rights to the book as a whole are reserved by INTECHOPEN LIMITED . The book as a whole (compilation) cannot be reproduced, distributed or used for commercial or non-commercial purposes without INTECHOPEN LIMITED's written permission. Enquiries concerning the use of the book should be directed to INTECHOPEN LIMITED rights and permissions department (permissions@intechopen.com).

Violations are liable to prosecution under the governing Copyright Law .

\section{(cc) BY}

Individual chapters of this publication are distributed under the terms of the Creative Commons Attribution 3. 0 Unported License which permits commercial use, distribution and reproduction of the individual chapters, provided the original author(s) and source publication are appropriately acknowledged. If so indicated, certain images may not be included under the Creative Commons license. In such cases users will need to obtain permission from the license holder to reproduce the material. More details and guidelines concerning content reuse and adaptation can be found at http : //www . intechopen . com/copyright-policy . html .

\section{Notice}

Statements and opinions expressed in the chapters are these of the individual contributors and not necessarily those of the editors or publisher. No responsibility is accepted for the accuracy of information contained in the published chapters. The publisher assumes no responsibility for any damage or injury to persons or property arising out of the use of any materials, instructions, methods or ideas contained in the book.

First published in London, United Kingdom, 2020 by IntechOpen IntechOpen is the global imprint of INTECHOPEN LIMITED, registered in England and Wales, registration number: 11086078 , 7th floor, 10 Lower Thames Street, London,

EC3R 6AF, United Kingdom

Printed in Croatia

British Library Cataloguing-in-Publication Data

A catalogue record for this book is available from the British Library

Additional hard and PDF copies can be obtained from orders@intechopen.com

Practical Applications of Electrocardiogram

Edited by Umashankar Lakshmanadoss

p. cm.

Print ISBN 978-1-78984- $077-3$

Online ISBN 978-1-78984-078-0

eBook (PDF) ISBN 978-1-78984-@83-4 


\section{We are IntechOpen, \\ the world's leading publisher of Open Access books}

\section{Built by scientists, for scientists}

\section{$4,600+$}

Open access books available

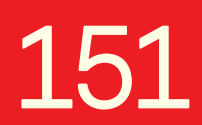

Countries delivered to

\section{$120,000+$}

International authors and editors

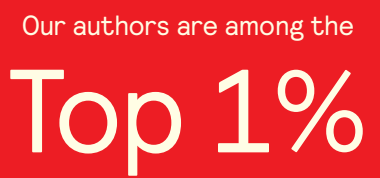

most cited scientists

Contributors from top 500 universities
$135 \mathrm{M}+$

Downloads
$12.2 \%$

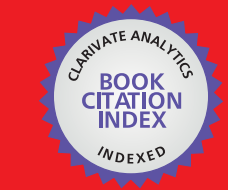

WEB OF SCIENCE ${ }^{\text {M }}$

Selection of our books indexed in the Book Citation Index in Web of Science ${ }^{\mathrm{TM}}$ Core Collection (BKCI)

\section{Interested in publishing with us? \\ Contact book.department@intechopen.com}

Numbers displayed above are based on latest data collected.

For more information visit www.intechopen.com 



\section{Meet the editor}

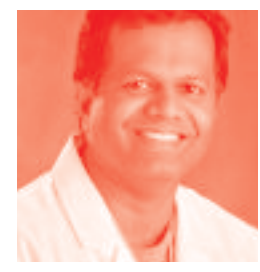

Dr. Umashankar Lakshmanadoss completed his training at the University of Rochester, NY, USA. He served as Director of the Inpatient Medical Consult Service at Johns Hopkins University School of Medicine and then joined the Division of Cardiovascular Medicine at Guthrie Clinic, Sayre, PA, USA. He then pursued training in cardiac electrophysiology at William Beaumont School of Medicine and continued advanced cardiac electrophysiology training at Mayo Clinic, Rochester, MN, USA. He served as an assistant professor of medicine in the Division of Cardiology, Louisiana State University, Shreveport, LA, USA, where he was also the director of the complex arrhythmia ablation program. His research interest is in the field of cardiac electrophysiology. Currently, he is the Director of Cardiac Electrophysiology at Mercy Health, Cincinnati, $\mathrm{OH}, \mathrm{USA}$. 



\section{Contents}

Preface

Section 1

Basic Science of Electrocardiography

Chapter 1

Diagnosing Abnormal Electrocardiogram (ECG) via Deep Learning by Xin Gao

Chapter 2

Combination of the CEEM Decomposition with Adaptive Noise and Periodogram Technique for ECG Signals Analysis

by Azzedine Dliou, Samir Elouaham, Rachid Latif and Mostafa Laaboubi

Chapter 3

Visualization of ECG Data on Variant Maps

by Zhihui Hou and Jeffrey Zheng

Section 2

Clinical Applications of Electrocardiography

Chapter 4

Characteristics of Atrial Premature Beat ECG Signals on Variant Maps by Lihua Leng, Jeffery Zheng and Jing Zhang

Chapter 5

Ventricular Tachycardia and Heart Failure

by Hakan Altay

Chapter 6

Genetic Polymorphisms that Playing Role in Development of Hypertrophic Cardiomyopathy by Nevra Alkanli and Arzu Ay 



\section{Preface}

Cardiovascular disease continues to be the leading cause of morbidity and mortality in both developed and developing countries. We all continue to struggle to have a better outcome from cardiovascular diseases. Early diagnosis and management of cardiovascular diseases play a major role to achieve this goal. However, appropriate diagnoses of cardiovascular diseases_-either ischemic heart disease or arrhythmiasremains a challenge. The electrocardiogram (ECG) plays a major role in diagnosing both ischemic heart disease and arrhythmia disorders. Hence, it plays a vital role in the field of cardiology.

This book provides an excellent overview of the diagnosis of abnormal ECGs, and is divided into two sections, including basic and practical applications of ECGs. In the first section, the authors provide an excellent discussion on the basic techniques of obtaining and processing electrocardiogram signals through deep learning methods; these are optimal techniques that can link the processing and analysis of nonstationary ECG signals, and the various statistical methods for converting ECG data into variant maps. In the second section, the authors provided a comprehensive review of the practical applications of ECGs. They discuss the application of various methods for identifying premature atrial beats, ECG characteristics of right and left ventricular tachyarrhythmia, and conditions producing left ventricular hypertrophy, including hypertrophic cardiomyopathy.

Although improvements in primary and secondary prevention of cardiac disease have resulted in a substantial reduction in overall mortality from cardiovascular diseases, the incidence and prevalence of sudden cardiac arrest have not declined. The most common cause of sudden cardiac arrest is ventricular tachycardia. It is common knowledge that congestive heart failure is a major public health problem worldwide. Patients with very low ejection fraction are at high risk of having ventricular tachycardia, which eventually leads to sudden cardiac arrest. In this book, we have an excellent review of the management of ventricular tachycardia in the setting of congestive heart failure. Similarly, hypertrophic cardiomyopathy is another cardiac disease that can produce sudden cardiac arrest in a relatively healthy population. This needs to be identified as soon as possible and action taken. Also provided is an excellent review addressing the genetic polymorphisms that play a major role in the development of hypertrophic cardiomyopathy. I sincerely believe that this will serve as a reference for clinicians who are involved in the complex cardiac care of these patients.

I gratefully acknowledge the invaluable organizational skills of the publisher IntechOpen, the timely and invaluable assistance of publishing process manager Ms. Rozmari Marijan, the design, technical generator, and information technology staff, and finally the marketing representatives who are working constantly to promote the book on various platforms. I sincerely appreciate and applaud all the contributing authors for their excellence, and hard work and commitment to the chapters. They have taken time from their personal and professional lives to complete this task. I thank them profusely for that. 
We hope that this book will serve as a reference for the techniques used to obtain and process electrical signals for ECGs. This book is dedicated to my mother Mrs. Jana (for her constant support, continued encouragement, and unconditional love) and to my little one Master Shawn who continues to inspire me and make my life blissful.

Umashankar Lakshmanadoss MD FHRS

Mercy Heart Institute,

Cincinnati, $\mathrm{OH}$, USA 
Section 1

\section{Basic Science of Electrocardiography}





\title{
Diagnosing Abnormal Electrocardiogram (ECG) via Deep Learning
}

\author{
Xin Gao
}

\begin{abstract}
In this chapter, we investigate the most recent automatic detecting algorithms on abnormal electrocardiogram (ECG) in a variety of cardiac arrhythmias. We present typical examples of a medical case study and technical applications related to diagnosing ECG, which include (i) a recently patented data classifier on the basis of deep learning model, (ii) a deep neural network scheme to diagnose variable types of arrhythmia through wearable ECG monitoring devices, and (iii) implementation of the health cloud platform, which consists of automatic detection, data mining, and classifying via the Android terminal module. Our work establishes a cross-area study, which relates artificial intelligence (AI), deep learning, cloud computing on huge amount of data to minishape ECG monitoring devices, and portable interaction platforms. Experimental results display the technical advantages such as saving cost, better reliability, and higher accuracy of deep learning-based models in contrast to conventional schemes on cardiac diagnosis.
\end{abstract}

Keywords: electrocardiogram (ECG), cardiac arrhythmia, deep learning, health cloud platform

\section{Introduction}

Statistical reports indicated that the leading cause of death in the world comes from cardiovascular diseases [9, 20]. The World Health Organization (WHO) reported that the total number of deaths from cardiovascular diseases in 2012 was approximately 17.5 million, compared with 17.7 million in 2015, and this number has been increasing every year $[1,3,9]$. With the accelerating pace of life, more and more young people suffer from great pressure related to work, and completely ignore physical examinations, which increases the risk of sudden death [9]. Hence, monitoring ECG and performing automatic diagnosis become particularly important. In cardiology, the electrical actions of a human's heart are simply and painlessly recorded by electrocardiogram (ECG) via single or multiple-lead detections $[8,20]$. The real-time ECG sequence of a patient represents one of the most useful clinical diagnostic features on cardiovascular diseases, reflecting the electrophysiological activity of cardiac excitement, and indicating great importance on the aspects of basic heart functions and related pathological research [12]. Meanwhile, ECG is of crucial importance for analyzing and identifying various arrhythmias, which reflect the degree of myocardial damage, the corresponding development process, and the functional structure of both atria and ventricles $[3,12]$. A few 
research scholars have related medical evidence of ECG toward arrhythmias with the latest experimental study, see [3, 4, 12, 23, 29], and the references therein.

Typical anomaly behaviors in ECG refer to irregular heartbeats, which are often recognized as sinus arrhythmia, ectopic tachycardia, cardiac flutter and tremor, and heart block [12]. The ectopic tachycardia is also known as premature contraction, standing for the most common types of cardiac arrhythmias. Classification of arrhythmias can be in accordance with a cardiac pacemaker and the conduction process $[9,12]$ : abnormal pacemakers may lead to arrhythmias and fluctuated heart rates, including atrial fibrillation, ventricular fibrillation (either sinus, supraventricular, or ventricular), tachycardia and bradycardia; abnormal cardiac conduction system results into heart blocks such as atrioventricular block and intraventricular block, etc. Atrioventricular block takes place in the atrioventricular node, the His bundle and its branches, while ventricular conduction block occurs in the left and right bundle branches. Specifically, no obvious symptoms appear in left bundle branch block, while coronary and rheumatic heart diseases as well as acute myocardial infarction often accompany right bundle branch block. Healthy people come along with occasional atrial premature beats, while ventricular premature beats are often associated with some kind of organic lesion. Several distortions on QRS wave and ST segments could appear in those cardiac arrhythmias [12].

Previously reported medications and medical procedures such as pacemaker insertion and surgery offer well-established treatments for most arrhythmias; meanwhile, a large quantity of signal and image processing algorithms as well as sensor devices provided useful tools on electrocardiogram-assisted diagnosis [8, $18,20,26,31,32]$. Recently, many researchers have been devoting themselves on computer-aided ECG analysis, where the technical developments are enriched from the booming growth on machine learning and deep learning algorithms $[6,9,11$, $13-17,21,24,25,27,30,33,35-37]$. Their methodology of study is broadly categorized as conventional machine learning and deep learning. Traditional machine learning schemes are greatly affected by data, which demands complex preprocessing such as noise removal and data normalization. Besides, it is also vulnerable to over-extract unnecessary features, requiring filter design and sorting out redundant features, and then finally input another algorithm for classification $[10,16,19,21$, 25-28]. While good effects on recognition got achieved, the overall procedure is more complicated than those of the deep learning schemes $[1,6,9,14,17,21,25$, 27]. Hence, previous machine learnings are no longer suitable to be embedded into mobile devices or perform real-time analysis [15].

For some of the deep learning-based schemes [1, 9, 13-15, 17, 25, 27], it is not only unnecessary to perform accurate denoising on the data, but also automatically extract the features in order to achieve the expected ideal recognition results. Common training modes of algorithmic ECG diagnosis usually compose single lead and multilead [9]. Multilead data combined with multichannel neural network algorithms (MCNN) are capable of achieving considerably good results; however, their shortcomings display on the relatively larger training parameters of models and much longer training time, which increases the difficulties to realize real-time monitoring associated with the existing mobile devices [9, 10, 31, 32]. Comparing to single-lead ECG data processing, the performance of recognition by multilead can be achieved with satisfactory, i.e., using the AlexNet structure, while the weakness lies on that these methods were regarded as relatively out of date [9].

The remainder of this chapter is organized as follows. In Section 2, several typical algorithms are briefly described on how deep learning-based light-quantity level algorithms recognize ECG data, and how the principles of deep learning are related on accurately diagnosing cardiac arrhythmia. Section 3 introduces a recently patented ECG data classifier with deep learning-based model. The automatic ECG 
arrhythmia diagnosing system and Android health cloud platform are referenced in Section 4. The last Section 5 prospects the progress on cross-area study of artificial intelligence-(AI)-related ECG diagnosis and draws our conclusions.

\section{Deep learning theory and automatic ECG diagnosis}

Since ECG periodically reflects the variations on electrical activities of a human's heart and real-time monitoring indicates parallel processing on amazing amount of data, automatic ECG diagnosis calls for efficient classification techniques on extracting unsupervised data features in practical ways. Many previous statistical signal processing or machine learning schemes utilize some dimensional reduction methods (i.e., linear discriminate analysis (LDA), independent component analysis (ICA), principle component analysis (PCA), etc.) to release the complexity issues, while it is the fact that practically most of the feature selection schemes are still dependent on human labor [9]. Deep learning is developed from artificial neural networks to simulate the input and output of neurons and the process of excitatory transmission of signals $[9,21,25]$. While early neural network (perceptron) only aimed to solve the linear separable problem, deep learning models connect some hidden layer(s) with an activation function between the input and output layers to obtain multilayer perceptions (MLP) [9]. Expanded cascades on the hidden layer of neurons indicate that the ability of network learning is deepened, and hence, any arbitrary continuous function of arbitrary complexity was proved to be effectively approached (in any level of accuracy), given an expression of a functional model, while the by-products turn to be increased network parameters and difficulty on training [9].

Two representative training methods in deep learning-based ECG diagnosis include the back-propagation (BP) algorithm and deep belief network (DBN) $[1,8,28]$, where the former still fails to overcome the error dissipation effect in the process of back-propagation, and the latter is suitable for layer-by-layer unsupervised learning via using a small portion of labeled samples for global optimization; for feature learning, DBN makes full use of unlabeled data and reduces the cost via the strategy of "pretraining and minor tuning" [9]. Other applicable deep network structures applied in latest works on automatic ECG analysis comprise fundamental or variation schemes related to classical MLP, convolutional neural networks $(\mathrm{CNN})$, and recurrent neural networks (RNN) [9, 22]. A schematic diagram of CNN-based arrhythmia classification is displayed in Figure 1 [9].

The three basic features of CNN, known as locally receptive field, shared weights and pooling, are reflected inside of the input and output layers in Figure 1 as depicted above [9]: the convolutional layer exploits sample information fragments in the form of moving windows (locally acceptable domain) to continuously learn the entire information of samples, and traverse to obtain multiple feature maps by weight sharing, which is the convolution layer. The pool layer performs data compression on the feature map of convolutional layer in order to simplify its output. Frequently used max-pool operation filters out all redundant values except the maximum value in the sample region, and then transforms the data to improve the algorithmic robustness. The upper fully layer corresponds to the network output, and the combination of convolution layer, and pool layer can also be inserted with full layers to acquire middle outputs. Since diagnosing ECG is also a task of time series analysis, its information is mainly expressed by the spatial structure, and the information output by each channel of the multilead ECG is not identically the same. The chest V1 lead signal and the limb II lead signal are both inputs into the neural network, and the output layer performs classification on different types of arrhythmia [9]. 


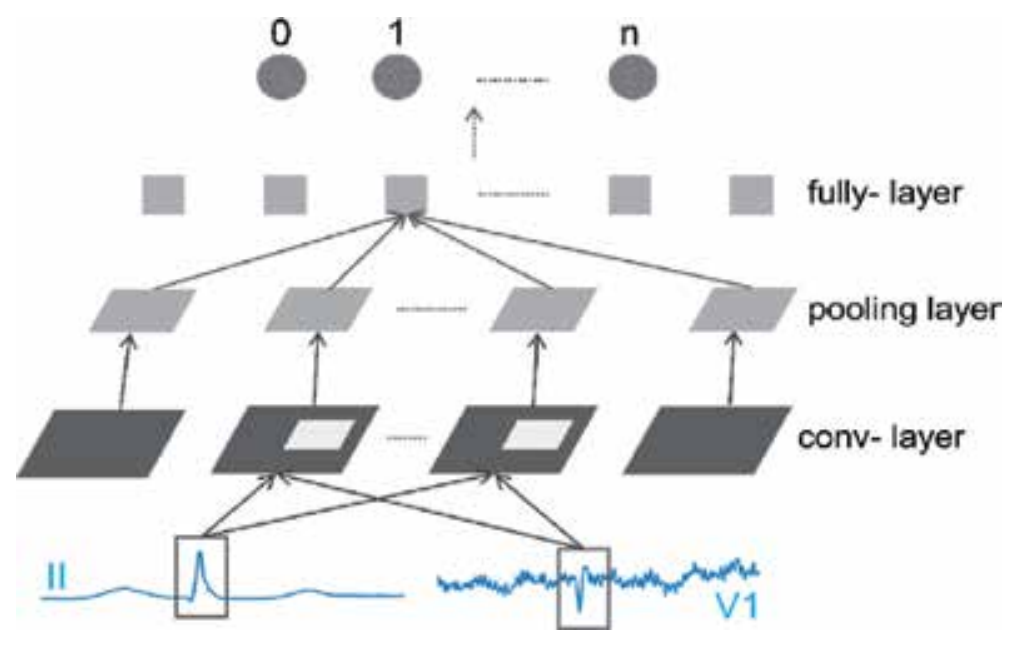

Figure 1.

A schematic diagram of CNN-based automatic arrhythmia classification from ECG [9].

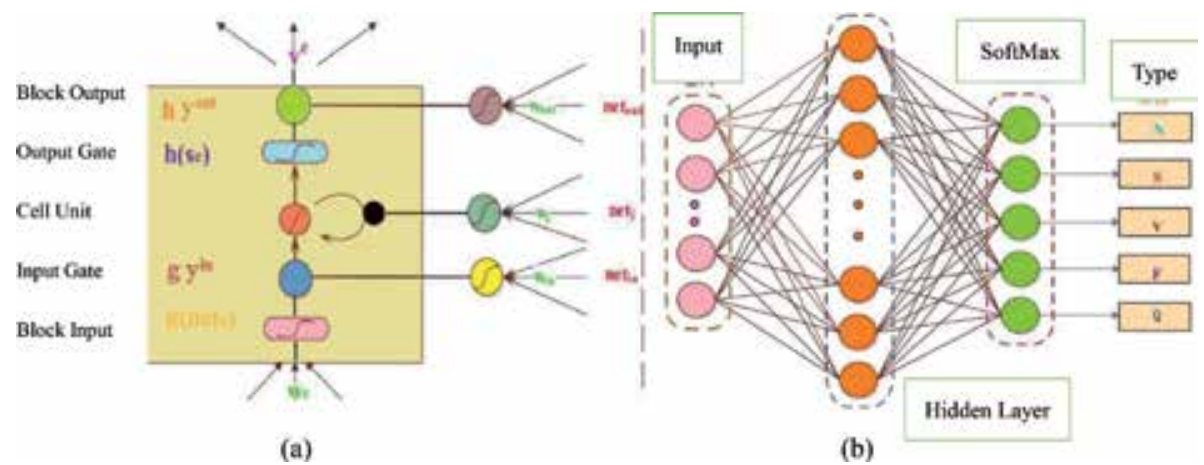

Figure 2.

LSTM-based single cell-unit model and schematic diagram for arrhythmia classification [18].

While applying deep learning theory in automatic ECG diagnosis and arrhythmia classification, in addition to BP algorithm, CNN and fully connected feedforward neural network (FCFNN), the gradient descent training algorithm also suggests a feasible candidate $[9,22]$. In the optimization process of training, the follow-up methods such as target function selection, dropout technique, and Nesterov impulse update are capable of improving the training efficiency and reduce the probability of over-fitting on sequential processing of ECG data [9, 22]. Regarding to RNN as mentioned above, its variation model named as long-short-term memory (LSTM) $[18,22]$ had been applied to classify arrhythmia, where the two share the same network structure, while the neurons in hidden layer got replaced with loop-connected memory units [18]. A standard memory unit contains single/multiple self-connected memory unit and three multiplication units (input/output gates and forgotten gate). Among the consecutive operations of "write," "read," and "reset," the forgotten gate offers a self-reset scheme for memory units, which is crucial to demand LSTM to "forgot" the previously loaded tasks [18]. A single cell-unit-based classical LSTM memory unit model is depicted in Figure 2a, and the schematic diagram of LSTMbased arrhythmia classification model is shown in Figure $2 \mathbf{b}$.

The LSTM-based model represents another deep learning scheme on diagnosing abnormal ECG and performing automatic arrhythmia classification [18]. With an input layer and two hidden layers, it cascades the SoftMax classifier as the last layer, 
which comprises five nodes that correspondingly stands for N, S, V, F, and Q [18]. Preprocessed ECG segments were taken as input data into LSTM to proceed with layer-to-layer feature learning and mapping, then the deep-level ECG signal features were sent to SoftMax classifier to perform training, from which the acquired weights follow-up with the initialization step and weight optimization step by BP algorithm so as to converge into the global optimal of LSTM network model, and finally achieve the goal of arrhythmia classification [18, 22]. The LSTM model overcame the dependence of traditional features on ECG signals, solved the problem of gradient elimination that early neural networks occur, and achieved data mining on the distinctive deep features behind large pool of ECG data via the proposed self-learning style [18].

In 2017, computer scientists in Stanford University claimed that they have developed a deep learning scheme on accurate diagnosing various types of arrhythmia, which achieved the grading level of diagnosing as high as professional cardiologists $[2,38]$. Such kind of deep learning-based schemes can sieve irregular heartbeats from sequential data of several hours. It is common to view arrhythmia from ECG, while doctors often supply patients with portable ECG that consecutively monitors their heartbeats since portable wearing devices are able to generate data in hundreds of hours [2]. Research scholars and a heartbeat monitoring company named as IRhythm have been working together to investigate accurate detection of deep CNN models toward large amount of concentrated, irregular ECG data [34, 38, 39]. It was claimed by these scholars that their proposed algorithm performed much better comparing to professional cardiologists when diagnosing 13 different types of arrhythmia [2]. Benefited from accelerating diagnosis and improving treatments, the algorithmic accuracy even exceeds those obtained by cardiological doctors. Besides, their ECG algorithm was expected to help people in remote areas gain some assistance from cardiological experts: performing some kind of anomaly detection, associated with processing various types of anomalous arrhythmia in high precision $[2,38]$. Applying their algorithm to monitor ECG of potential arrhythmia patients can be imaged in the following scenarios: when patients first come to see the doctor in office, if the wearable ECG device does not detect any problem, doctors would possibly allow the potential patient to use portable devices and monitor heartbeats consecutively for 2 weeks; hence, the crossover range of time generating data by the device is longer than 300 hours. After the second appointment, doctors may analysis the data of every second to discover any hint on arrhythmia [2].

Analyzing arrhythmia was in fact a data processing problem, as was found by Dr. Andrew Ng, a well-known artificial intelligence (AI) expert leading Stanford's machine learning team, where the deep learning algorithm they developed aims to diagnose different types of arrhythmias from ECG inputs [2]. Cooperating with companies, which provide wearable rhythm monitoring equipment, about 36,000 ECG data samples were acquired to train a deep neural network model, which was later proved to be more accurate than a cardiologist in diagnosing arrhythmias, and performs even better than a doctor in most cases $[2,38]$. Their trained 34-layer CNN model is depicted in Figure 3, where a single-lead wearable heart device monitors ECG, and the objective is oriented on correct detection of the sinus rhythm (SINUS) and atrial fibrillation (AFIB). The input after preactivation, followed by 33 convolution layers in cascades, one fully connected layer at the last and a SoftMax, contributed the entire architecture of this trained deep neural network [38].

Research scholars discovered that many types of arrhythmia are similar on occurrence, while their differences are trivial; however, it has a great impact on how to deal with a specific arrhythmia: for instance, two types of arrhythmias were known as secondary atrioventricular block and showed very similar appearance, while one requires no treatment and the other urges immediate observation [2, 12, 22]. Their research products are not only able to discover signs of arrhythmia, but also expose different types of arrhythmia with unprecedented high precision [22]. The advantage 


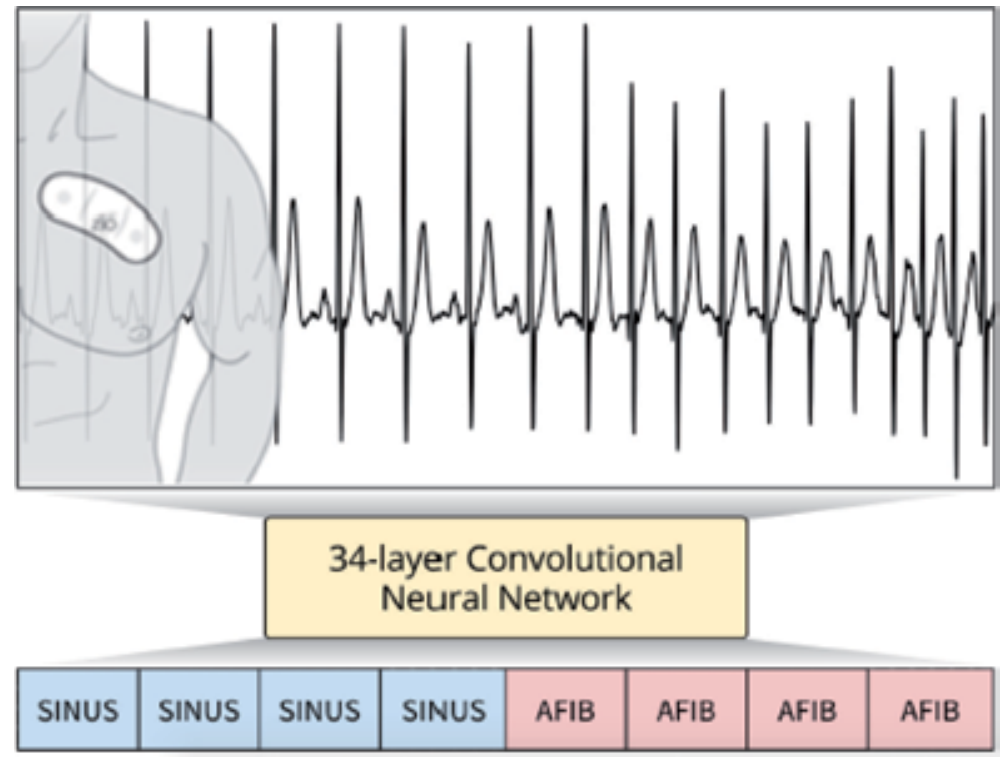

Figure 3.

The trained 34-layer CNN on arrhythmia detection on time-series ECG of random length [38].

of this deep CNN-based algorithm lies that it never become exhausted and continuously performs immediate diagnosis of arrhythmia, which further benefits patients who are unable to see a cardiologist in remote areas or a developing country [2]. When a potentially fatal heart rhythm appears in high-risk groups, one who wears a dailyused rhythm monitoring device will immediately respond and notify emergency personnel to aid the individual(s) with professional arrhythmia diagnosis [2,38].

\section{Automatic ECG diagnosis via deep learning: lightweight classifier}

Among the architecture model of deep learning-based schemes on automatic ECG diagnosis, reducing the computational cost, network parameters and training difficulties, will represent crucial problems. In order to solve these issues mentioned above, research scholars have been seeking for an ideal technical solution. A deep learning research lab in Zhengzhou University established a lightweight algorithm on automatic ECG data diagnosis, in which the elements on technical realization are displayed as below [40]: the objective is to provide a deep learning-based lightweight algorithm for identifying ECG data, which aims at the deficiencies of the current techniques. This invention takes along with a technical plan of identifying the ECG data based on deep learning, which includes the following steps [40]:

Step 1: Perform rough extraction of data features. The extracted ECG data are conveyed through a standard convolution layer

Step 2: Pass the rough extracted data features through a pooling layer max-pooling, then send these features to the core Lite module to extract deep-level data features

Step 3: Send the deep-level data features through a pooling layer max-pooling, then the two full-connection layers, named as dense, will receive these features by turns and perform purification

Step 4: Transmit the purified data features to the classifier function and proceed with outputs after feature classification.

A flowchart on the operating procedures with respect to this invention is depicted in Figure 4, and the diagram of its core module, the deep learning-based light weight algorithm, is shown in Figure 5. 


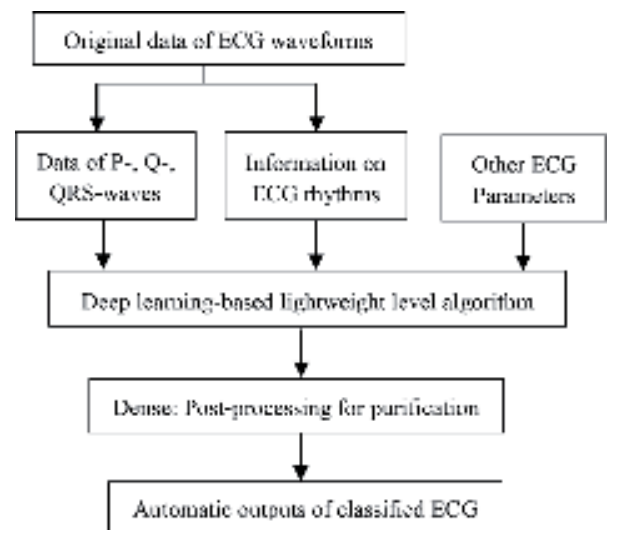

Figure 4.

The flowchart of automatic ECG classifier.

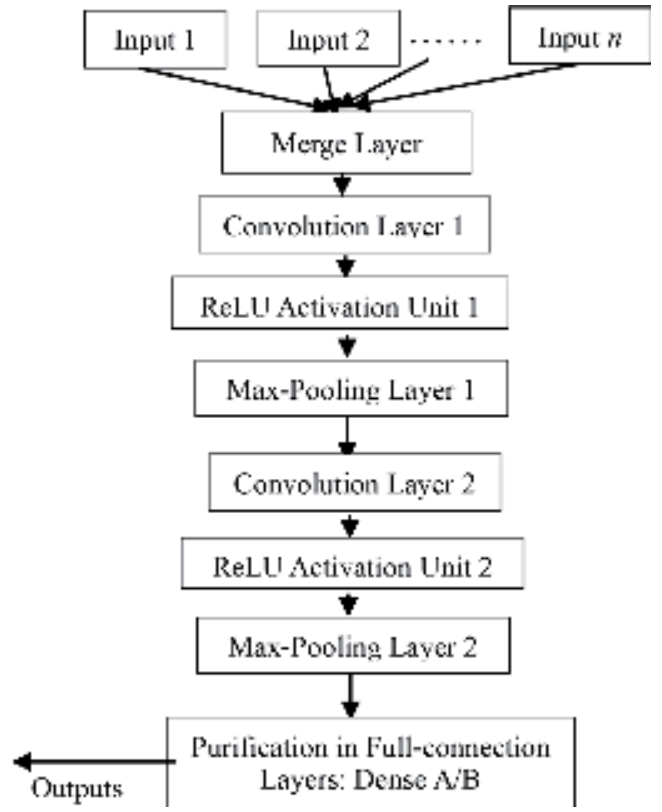

Figure 5.

Core modules of deep-learning based lightweight algorithm.

Regarding to the core module as proposed on invention, the max-pooling layer plays an anti-overfitting effect in the entire structure model and ensures the classification accuracy. The activation function of each convolutional layer including the fully connected layer is named as LeakReLU [40]. When performing the optimization step by the Adam optimizer, the learning rate was set as 0.001 . The specific setting parameters of this algorithmic model are presented as below.

Based on the analysis as described above, the activation functions of each convolutional layer and fully connected layer are implemented by LeakReLU, while the model is optimized using the Adam optimizer [40]: the learning rate is set as 0.001. In Step 1, the convolution kernel of the standard convolutional layer is set as $1 \times 5$ with step size of 1 ; the convolution kernel size of the pooled layer maxpooling is set as $1 \times 2$, with step size of 2 ; the convolution kernel size of the squeeze convolution layer and the first standard convolution layer are both set as $1 \times 1$ with step size of 1; similarly, with the same step size, convolution kernels of the second 
and third standard convolutional layers are set as $1 \times 2$ and $1 \times 3$, respectively; the convolution kernels of the depthwise convolutional layers match the same size with their follow-up standard convolution layers, their step sizes are set as 1 ; and the convolution kernel of the pointwise convolution layer is also set as $1 \times 1$ with step size of 1.

In Step 2, the function of core module lite is to install the squeeze convolution layer on compressing feature data outputted by the upper layer; after the layer of squeezed convolutions, the standard convolutions of three different channels were set to extract data features of different local sizes; the rough data features are transmitted through a depthwise convolution layer and a pointwise convolution layer after the second and the third standard convolutional layers; a residual connection is also constructed on the terminal right side of the squeeze convolution layer; finally, the outputs of filter concatenation are performed after the first standard convolution layer, pointwise convolution layer, and the residual connections [40].

It should be noted that the standard convolution layer in the invented automatic ECG classifier, performs a conventional convolution operation, which is named as standard convolutional layer for distinctions; the compression convolution layer is obtained by compressing the amount of feature data in the upper layer so as to reduce the computational load for convolution operation of the next layer. For example, if the upper layer outputs 10 feature data and the compressed convolution layer sets up five convolution kernels, then 5 feature data will be released, which means the input of next layer has five feature data. Deep convolution and pointwise convolution actually divide the ordinary standard convolution operation into two steps [40]: the first step is to exploit the deep convolution layer to perform convolution operations separately on each feature data of the previous layer, which indicates that a convolution kernel only convolves one feature data. In the second step, the pointwise convolution, which is the $1 \times 1$ convolution kernel, performs feature combination operations on the output after deep convolution.

By adopting the invented deep learning-based lightweight algorithm and through the setting of a core module, this model is trained to guarantee a certain accuracy in absence of demanding much computational cost. In contrast to other detection algorithms, the proposed scheme takes less time, displays faster prediction, and reduces personal consumption. The well-trained software platform can be embedded into a wearable ECG device or the mobile phone terminals to classify and monitor the collected ECG data, and triggers an alarm in case an abnormality is encountered. The embedded device is not only free of spending extra time affecting daily work, but also arouses people to pay enough attention on cardiac abnormalities and regularly perform physical examination; hence, the incidence of heart disease can be significantly reduced [40].

Comparing to the existing methods, this deep learning-based lightweight algorithm on invention has substantive features and significant advances, which can be specifically referred as [40]:

a. Core module stands for an innovative design module of structural fusion, which combines multilayer convolution kernel structure of the famous GoogleNet, the compressive convolution idea of SqueezeNet, and the depth-pointwise convolution on parameter reduction of MobileNets. By implementing the classical AlexNet network structure, the entire framework was designed to ensure the stability of algorithmic model.

b. Compared with other algorithms, this invented model does not require many computational parameters while ensures certain recognition effects; it also has the capacity on automatically realizing the processing of sequential ECG data on limited network resources or running memory. 
As specified by several steps mentioned above, the lightweight algorithm for identifying ECG data based on deep learning can be realized via uniprocessors in parallel computing, in which the convolution manner of each convolution layer is convolution of one-dimensional ECG data [40]. Brief notation on implementation is presented as follows: $x[n]$ and $y[n]$ denote the input and the output sequences, respectively; $h[n]$ represents the convolution kernel weight sequence, $h[-k]$ represents the inversion of the $h[k]$ sequence, $h[N-k]$ indicates that $h[-k]$ is moved by $n$ points; $m$ stands for the length of input sequence, while the length of output sequence is expressed as $\operatorname{len}(x[n])+\operatorname{len}(h[n])-1$. Because the classifier function is a SoftMax function classifying five types of ECG data, each type of ECG data is recorded as a neuron in the SoftMax function, which appears in the form as a product of the upper neuron output and the weight of SoftMax function connected to the upper neuron [40]. Hence, by constructing the loss function and the linear regression model, the output probability of each neuron and each neural unit can be modeled through vector calculus and probabilistic interpretation, which finally achieves the prediction value of output.

To sum up, the technical invention [40] provides a deep learning-based lightweight algorithm for automatic ECG data identification and diagnosis, where its procedure consists of extracting the extracted ECG data through a standard convolution layer, and performing rough extraction of data features; while a feature is passed through a max-pooling layer, the core module is sent to the kernel lite module to extract deep data features. Note that after passing the deep data features through a pooled layer max-pooling, those features will be sequentially loaded into two fully connected layers named as dense, in which purification is performed on the hierarchical data features; in the next step, the classifier function takes responsibility for feature classification on the output of purified data features. Compared with other similar schemes on automatic ECG diagnosis [5, 7, 16, 27, 31, 36], the invented lightweight algorithm has been released from requiring large set of calculation parameters but still ensures constant accuracy on recognition effects, which is able to realize parallel processing of ECG data despite of limited network resources or running memory on GPU [40].

\section{Automatic detection system for arrhythmias and the Android health cloud platform}

While the current methods on network medical treatments are often restricted to the interactions between doctors and patients via modern communication tools, it is neither possible to establish mutual trust nor collect real-time data. Hence, wearable ECG monitoring systems and applicable software platforms (typically integrated into Android terminal modules in a cellphone, for daily use) are calling for proposal. Recently, the health cloud platform for arrhythmia detection [9] and the ThingSpeak cloud computing platform [41] on classifying and diagnosing ECG had been proposed to solve some prior problems at different levels. Utilizing deep learning tools and the intelligent information integration platform named as the Internet of Things (IoT) [9, 41], a new follow-up mode of automatic heart monitoring system was developed for real-time remote services among doctors and patients in a long term. The system established a cloud platform on health inquiry, providing medical data management services for online patients. Aiming at reducing potential risks for cardiovascular diseases, this platform offers online assessment, diagnosis, and rehabilitation guidance by relevant doctors [9]. Regarding to offline services, this system relies on existing medical-level biosensors to construct terminals on signal acquisition and processing [9]. For real-time ECG sequence, it provides functions such as collection, exhibition, and analytical monitoring of basic physiological 


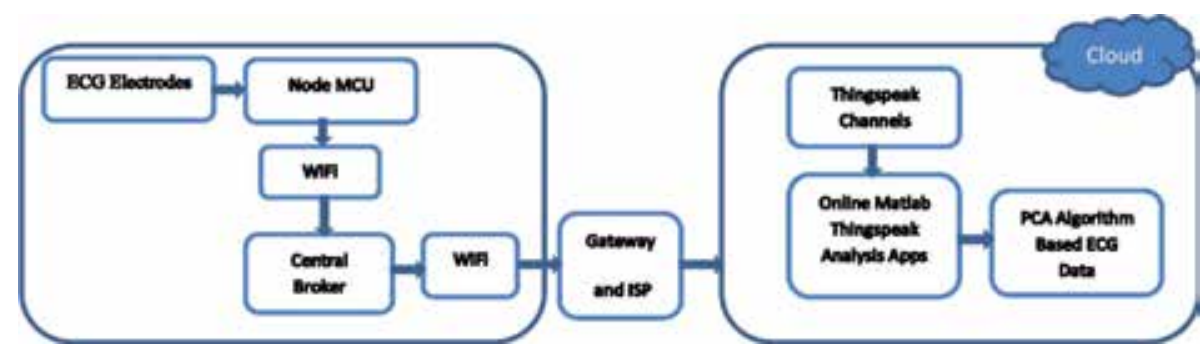

Figure 6.

The proposed ThingSpeak online monitoring system for ECG analytics using PCA [41].

parameters, and constructs a communication platform for doctors and patients with respect to their practical demands. The deep learning-based detection algorithm was integrated into the arrhythmia automatic diagnosis system within the module of health monitoring control, which performs real-time remote surveillance and outputs feedbacks on the ECG signals collected by the biosensors [9].

The system hardware mainly comprises a monitor system and an Android terminal [9]: a monitor system module is responsible for measuring and collecting data of vital signs such as ECG, blood pressure and body temperature, etc., then transmitting real-time data to the Android terminal module via serial port or Bluetooth and thereby completing the tasks of uploading data and synchronous exhibition, followed by performing automatic diagnosis (including real-time analysis) of possible arrhythmia data in the ECG sequence. The hardware of system monitor module adopts PM6750 for medical signal processing. RK3188 development motherboard with quad-core Cortex-A9 processor was chosen for hardware design on the Android terminal module. Original ECG data were sent to the terminal through the monitoring devices where the mode of asynchronous serial port transmission is applied, no parity bit exists, and the baud rate is 115,200 Baud. According to the protocol, Android terminal module parses the restored signal data including waveforms, heart rate and breathing, styles of single-lead or multilead, filtering, and signal gain. The parsed data can be uploaded to the cloud platform and saved as private health data for each client. Historical data can be viewed by each individual at any time through the browser to provide health channels and follow-up support on clinical treatments [9]. While ThingSpeak employed similar datasets on MIT database for ECG data, the proposed online monitoring system displays comparable outputs on ECG signals using principle component analysis (PCA), which is depicted in Figure 6 [41]. The online MATLAB programs are running through the ThingSpeak IoT cloud for automatic ECG data analysis, which enables doctors to monitor, diagnose, and improve the health of patients [41]; meanwhile, the call for emergency service ensures local first-aid institutions to respond at prompt time in order to minimize any risk issues in absence of proper treatments [9, 41].

In another scenario on the workflow of system, the automatic arrhythmia detection system starts with a network of bio-sensors, where the input signals follow the arranged entry to the bio-data acquisition module followed by the control module on health monitoring, and then uploaded into the cardiovascular health cloud platform for data analysis in Android systems [9]. Those mobile devices provide supplemental aid on building up virtual human models in digital physiological bases, simulating medical plans on treatment and predicting potential risks on disease. With the helpful support of remote clinical diagnosis in collaboration of artificial intelligence-based solutions such as electronic health and digital medicine plans, the comprehensive online medical cloud platform will come into reality very soon [9]. For broader applications on biomedical data management and access, Navale and Bourne [42] proposed a conceptual framework to show how the data 


\section{Conceptual Framework for Biomedical data Management and Access}

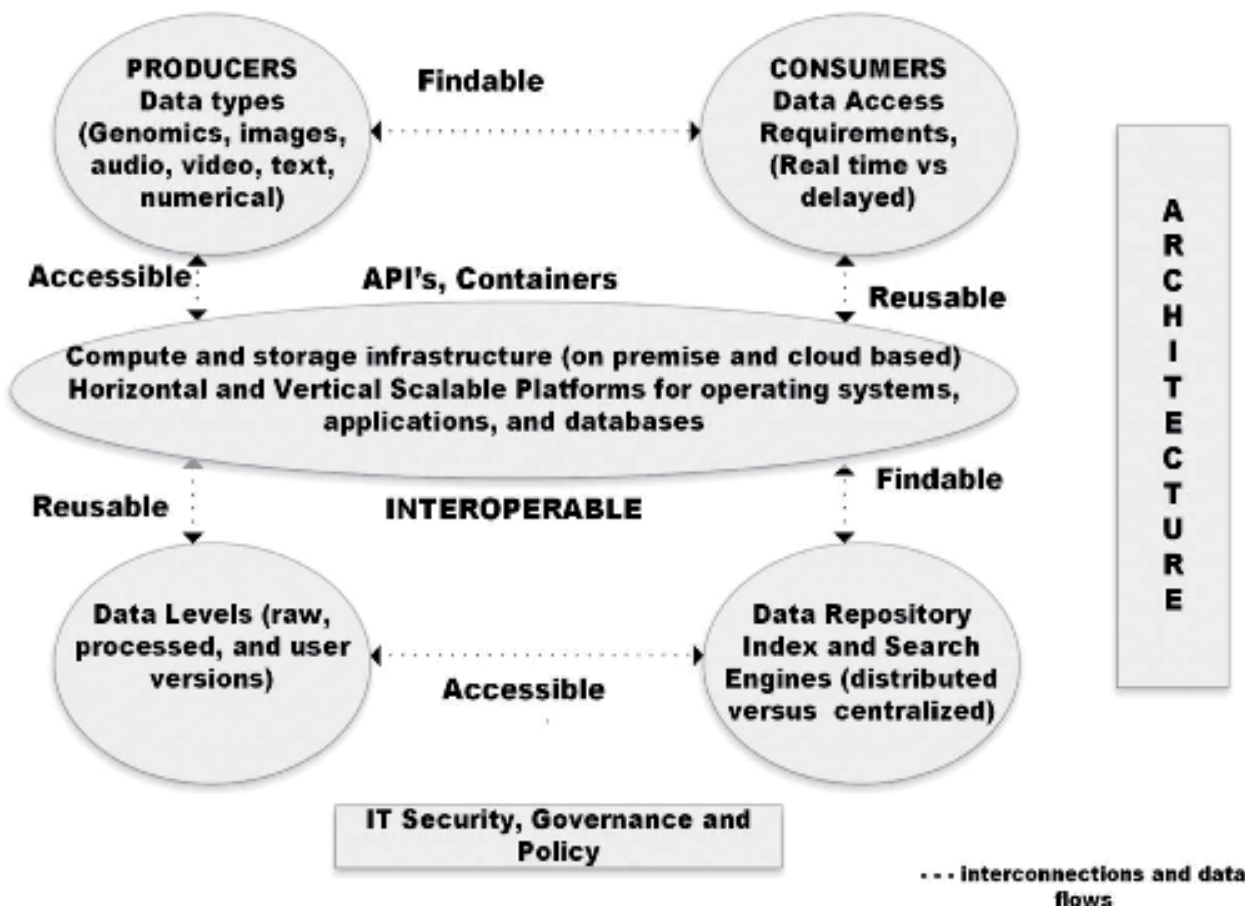

Figure 7.

The architecture of conceptual framework on cloud-based platform: variable data types flow between producers and consumers in support of the FAIR principle and in demands of different data level needs [42].

producers, consumers and repositories got integrated via a cloud-based platform; meanwhile, the open data and analytics must follow the principle of findable, accessible, interoperable and reusable (FAIR) to upgrade the discovery and innovation of knowledge. The architecture-based conceptual framework was depicted in Figure 7, where API stands for applicable programming interface [42].

\section{Discussions and conclusions}

In this chapter, we have established a study on deep learning theory related to automatic diagnosis on abnormal electrocardiogram (ECG). We briefly introduced the most recent automatic detecting schemes such as convolutional neural networks $(\mathrm{CNN})$, recurrent neural networks (RNN) [9, 22, 34, 39], and its variation of longshort-term memory (LSTM) model $[18,22]$, which aims on analyzing different types of cardiac arrhythmias. We presented an investigation of practical examples and applications of deep learning on automatic ECG diagnosis [5, 7, 16, 27, 31, 36], which consists of a deep learning-based lightweight classifier on ECG data identification, deep belief network (DBN) $[1,8,28]$ on diagnosing cardiac arrhythmia via wearable ECG monitoring devices, and a health cloud platform on automatic ECG detection, data mining and classification. We combined the theoretical concepts of artificial intelligence (AI)-oriented topics such as deep learning, big data health cloud platform to real medical applications, i.e., minishape ECG monitoring devices $[9,41]$, domestic cardiac arrhythmia analyzer [40], automatic ECG diagnosis on 
Android terminal modules [9] and the conceptual framework on managing and accessing biomedical data [42]. Technical advantages such as low-power consumption, higher accuracy, better reliability, and cost saving on the links of feasible software/hardware implementations to automatic cardiac arrhythmia diagnosis prospects broader applications of deep learning on ECG and other data analytics on medical imaging.

\section{Author details}

Xin Gao

Department of Electrical and Computer Engineering, The University of Arizona, Tucson, AZ, USA

*Address all correspondence to: xgao1985@email.arizona.edu

\section{IntechOpen}

(C) 2019 The Author(s). Licensee IntechOpen. This chapter is distributed under the terms of the Creative Commons Attribution License (http://creativecommons.org/licenses/ by/3.0), which permits unrestricted use, distribution, and reproduction in any medium, provided the original work is properly cited. (cc) BY 


\section{References}

[1] Altan G, Allahverdi N, Kutlu Y. A multistage deep learning algorithm for detecting arrhythmia. 1st International Conference on Computer Applications \& Information Security (ICCAIS). Riyadh: 2018. pp. 1-5; DOI: 10.1109/ CAIS.2018.8441942

[2] Anonymous. Professors in Stanford University of USA developed a new algorithm on diagnosing 13 types of arrhythmias. Journal of Practical Electrocardiology. 2017;26(4):246

[3] Behzadi M, Joukar S, Beik A. Opioids and cardiac arrhythmia: a literature review. Medical Principles and Practice. 2018;27:401-414

[4] Bravo-Valenzuela NJ, Rocha LA, Nardozza LMM, Júnior EA. Fetal cardiac arrhythmias: current evidence. Annals of Pediatric Cardiology. MayAug 2018;11(2):148-163

[5] Chen KM, Powers LS, Roveda JM. Noise-invariant component analysis for wearable sensor based electrocardiogram monitoring system. SM Journal of Biomedical Engineering. 2018;4(1):1025

[6] Chen YM, Gao X. The latest progress of deep learning. Computer Science and Applications. 2018;8(4):565-571. DOI: 10.12677/csa.2018.84064

[7] Fan XM, Yao QH, Cai YP, Miao F, Sun FM, Li Y. Multi-scaled fusion of deep convolutional neural networks for screening atrial fibrillation from single lead short ECG recordings. IEEE Journal of Biomedical and Health Informatics. 2018;22(6):1744-1753

[8] Gao X. Non-invasive detection and compression of fetal electrocardiogram. Interpreting Cardiac Electrograms From Skin to Endocardium, 2017: 53-74. http://dx.doi.org/10.5772/ intechopen. 69920
[9] Gao Y. Deep learning based automatic detection of arrhythmia and its applications. Thesis in Shandong University of Science and Technology, 2016:1-61

[10] Hu S, Wei HX, Chen YD, Tan JD. A real-time cardiac arrhythmia classification system with wearable sensor networks. Sensors. 2012;12:12844-12869. DOI:10.3390/ s120912844

[11] Hu X, Yu ZB. Diagnosis of mesothelioma with deep learning. Oncology Letters. Oct 2018:1-8. DOI: 10.3892/ol.2018.9761

[12] Hsing MJ, Hsia HH, Cardiac Arrhythmias. 2010:341-374. DOI: 10.1007/978-0-387-77452-7_19

[13] Isin A, Ozdalili S. Cardiac arrhythmia detection using deep learning. Procedia Computer Science. 2017;120:268-275

[14] Jin LP, Dong J. Deep learning research on clinical electrocardiogram analysis. China Science: Information Science. 2015;45(3):398-416

[15] Jin LP, Dong J. Normal versus abnormal ECG classification by the aid of deep learning. Artificial Intelligence Emerging Trends and Applications. InTech Open; 2018. pp. 295-315

[16] Kamaleswaran R, Mahajan R, Akbilgic $O$. A robust deep convolutional neural network for the classification of abnormal cardiac rhythm using varying length single lead electrocardiogram. Physiological Measurement. Jan 2018: 1-12. DOI: 10.1088/1361-6579/aaaa9d

[17] Lee YN, Kwon JM, Lee YH, Park $\mathrm{HH}$, Cho H, Park JS. Deep learning in the medical domain: predicting cardiac arrest using deep learning. Acute and Critical Care. 2018;33(3):117-120 
[18] Li X. Research of arrhythmia classification based on LSTM. Thesis in Lanzhou University. 2018:1-58

[19] Kalidas V, Tamil LS. Cardiac arrhythmia classification using multimodal signal analysis. Physiological Measurement. 2016;37:1253-1272. DOI: $10.1088 / 0967-3334 / 37 / 8 / 1253$

[20] Kalra A, Lowe A, Al-Jumaily A. Critical review of electrocardiography measurement systems and technology. Nov 2018. DOI: 10.1088/1361-6501/ aaf2b7

[21] Krishna MM, Neelima M, Harshali M, Rao MVG. Image classification using deep learning. International Journal of Engineering \& Technology. 2018;7(2.7):614-617

[22] Oh SL, Ng EYK, Tan RS, Acharya UR. Automated diagnosis of arrhythmia using combination of CNN and LSTM techniques with variable length heart beats. Computers in Biology and Medicine. June 2018. pp. 1-24. DOI: 10.1016/j.compbiomed.2018.06.002

[23] Papadopoulos CH, Oikonomidis D, Lazaris E, Nihoyannopoulos P. Echocardiography and cardiac arrhythmias, Hellenic Journal of Cardiology. 2017:1-10. https://doi. org/10.1016/j.hjc.2017.11.017

[24] Pourbabaee B, Roshtkhari MJ, Khorasani K. Deep convolutional neural networks and learning ECG features for screening paroxysmal atrial fibrillation patients. IEEE Transactions on Systems, Man, and Cybernetics: Systems. 2018;48(12):2095-2104

[25] Pyakillya B, Kazachenko N, Mikhailovsky N. Deep learning for ECG classification. Journal of Physics: Conf. Series. 2017;913:012004. DOI: 10.1088/1742-6596/913/1/012004

[26] Rajagopal R, Ranganathan V. Critical evaluation of linear dimensionality reduction techniques for cardiac arrhythmia classification. Circuits and Systems. 2016;7:2603-2612

[27] Sanninoa G, Pietroa GD. Deep learning approach for ECG-based heartbeat classification for arrhythmia detection. Journal of Future Generation Computer Systems. 2018

[28] Savalia S, Emamian V. Cardiac arrhythmia classification by multi-layer perceptron and convolution neural networks. Bioengineering. 2018;5(35): 1-12. DOI:10.3390/bioengineering5020035

[29] Shahreyar M, Fahhoum R, Akinseye O, Bhandari S, Dang G, Khouzam RN. Severe sepsis and cardiac arrhythmias. Annuals of Translational Medicine. 2018;6(1). DOI: 10.21037/atm.2017.12.26

[30] Sun TK, Bao R, Jiang DH, Wang K. Security technology of the big cloud data based on deep learning. Computer Science and Applications. 2015;5(9):336-342

[31] Xia YF, Gao ZF, Zhang HL, Zhang HY, Li S. An automatic cardiac arrhythmia classification system with wearable electrocardiogram. IEEE Access. 2018. DOI: 10.1109/ ACCESS.2018.2807700

[32] Xia YF, Zhang HL, Xu L, Gao ZF, Zhang HY, Liu HF, Li S. An automatic cardiac arrhythmia classification system with wearable electrocardiogram. IEEE Access. 2018;6:165529-16538

[33] Yap J, Yolland W, Tschandl P. Multimodal skin lesion classification using deep learning. Experimental Dermatology. 2018;00:1-7. https://doi. org/10.1111/exd.13777

[34] Zhan YJ, Wang J, Shi JP, Cheng GL, Yao LL, Sun WD. Distinguishing cloud and snow in satellite images via deep convolutional network. IEEE Geoscience and Remote Sensing Letters. 2017;14(10):1485-1489 
[35] Zhang R, Li WP, Tong M. Review of deep learning. Information and Control. 2018;47(4):385-397

[36] Zhong W, Liao LJ, Guo XM, Wang GL. A deep learning approach for fetal QRS complex detection, Physiological Measurement. 2018;39(4):045004(9pp). DOI: 10.1088/1361-6579/aab297

[37] Zhu YL, Lu ST, Dai RC, Liu GY, Wang ZW. Power market price forecasting via deep learning. 44th Annual Conference of the IEEE. Washington DC, USA: Industrial Electronics Society (IECON); 21-23 Oct 2018. https://arxiv.org/abs/1809.08092

[38] Rajpurkar P, Hannun AY, Haghpanahi M, Bourn C, Ng, AY. Cardiologist-level arrhythmia detection with convolutional neural networks. [Online] Available: arxiv.org/ pdf/1707.01836.pdf

[39] Ribeiro AH, Ribeiro MH, Paixão G, Oliveira D, Gomes PR, Canazart JA, Pifano M, Meira-Jr. W, Schön TB, Ribeiro AL. Automatic diagnosis of short-duration 12-lead ECG using a deep convolutional network. [Online] Available: arxiv.org/pdf/1811.12194. pdf

[40] A deep learning-based lightweight algorithm for identifying ECG data. [Online] Available: http://www.xjishu. com/zhuanli/55/201810353923.html

[41] Thingspeak cloud computing platform based ECG diagnose system. [Online] Available: http://www.researchgate.net/ publication/328042628_Thingspeak_ Cloud_Computing_Platform_Based_ ECG_Diagnose_System

[42] Navale V and Bourne PE.

Cloud computing applications for biomedical science: a perspective. PLoS Computational Biology. 2018;14(6):e1006144. https://doi. org/10.1371/journal.pcbi.1006144 



\title{
Combination of the CEEM \\ Decomposition with Adaptive Noise and Periodogram Technique for ECG Signals Analysis
}

\author{
Azzedine Dliou, Samir Elouaham, Rachid Latif and \\ Mostafa Laaboubi
}

\begin{abstract}
The electrocardiogram (ECG) signal is a fundamental tool for patient treatment, especially in the cardiology domain, due to the high mortality rate of heart diseases. The main objective of this paper is to present the most optimal techniques that can link the processing and analysis of ECG signals. This work is divided into two steps. In the first one, we propose a comparison between some denoising techniques that can reduce noise affecting the ECG signals; these techniques are the empirical mode decomposition (EMD), the ensemble empirical mode decomposition (EEMD), and the complete ensemble empirical mode decomposition with adaptive noise (CEEMDAN). In the second one, we make a comparison of three time-frequency techniques: the Choi-Williams (CW), the periodogram (PE), and the smoothed pseudo Wigner-Ville (SPWV). Firstly, the obtained results illustrate the effectiveness of the CEEMDAN in reducing noise that interferes with ECG signals compared to other denoising methods. Secondly, they show that the periodogram time-frequency technique gives a good detection and localization of the main components in the time-frequency plan of ECG signals. This work proves the utility of the combination of the periodogram and CEEMDAN techniques in analyzing the ECG signals.
\end{abstract}

Keywords: ECG, CEEMDAN, periodogram, time-frequency, denoising

\section{Introduction}

The heart function can be obtained by storing the voltage variations which occur on some parts of the human body surface [1-3]. The electrocardiogram (ECG) is the record of those voltage variations over time. This biomedical signal presents a fundamental tool used in cardiology to detect cardiac diseases. The normal ECG signal is characterized by a sequence of some well-defined components as $\mathrm{P}$ wave, QRS complex, and T wave [1-3]. ECG signals are most of the time contaminated by different noise sources, like power-line interference, baseline wander, muscle noise and motion artifact, and other noises, which in different cases make the 
identification of standard ECG features very difficult and lead to a misjudgment of patient diagnostic [4]. Consequently, to deal with this problem, a task of removing noise from ECG signal, as preprocessing step, has become very important.

To tackle this problem, the first part of the current work proposes a comparison study of the following denoising methods, empirical mode decomposition (EMD), ensemble empirical mode decomposition (EEMD), and complete ensemble empirical mode decomposition with adaptive noise (CEEMDAN), to define which one gives the best results in the case of the normal and abnormal ECG signals.

Huang et al. [5] have introduced the empirical mode decomposition (EMD) method to analyze nonstationary and nonlinear signals. The EMD major advantage is that the basic functions are derived from the signal itself; however, the EMD process presents a mode mixing. To surmount this problem, we resort to ensemble empirical mode decomposition (EEMD); this denoising method employs EMD to integrated signals with white Gaussian noise [6]. Even so, signals with added noise can produce a large number of iterations in the EEMD process, and signal result holds residual noise after decomposition. These downsides are resolved with a variant denoising method, called CEEMDAN; this technique achieves an accurate original signal reconstruction. The CEEMDAN iteration number is minus than half of the EEMD iteration number [7].

Traditionally, ECG signal, are analyzed in the time domain by skilled physicians. However, detecting pathological conditions in the time domain is not always evident [8]. The precision and the exactitude of the diagnosis are in relation with the cardiologist experience and the concentration rate.

This fact has incentive applying the frequency domain techniques, such as Fourier transform (FT) analysis [9]. The development of the Cooley-Tukey algorithm made Fourier techniques widely available; this algorithm allows the use of the computation more efficient [10]. However, the ECG signals are multicomponent nonstationary signals [8]; accurate time-varying spectral estimates can be extremely difficult to obtain with Fourier techniques which give only globally averaged information.

To overcome this problem, time-frequency techniques can be a good solution. These techniques can reveal the multicomponent nature of such signals and how the signal spectrum evolves over time [11-13].

Time-frequency techniques can be classified into two major categories: parametric and nonparametric techniques. Nonparametric time-frequency techniques present a good solution for analyzing multicomponent nonstationary signal [13-15]. However, these techniques suffer from the presence of cross-terms [16-18], which can hide the interesting signal information. A lot of efforts have been made to select the best timefrequency technique which provides a low degree of cross-term effect [13-18].

The second part of the this work is consecrated to compare three time-frequency techniques, Choi-Williams (CW), periodogram (PE), and smoothed pseudo Wigner-Ville (SPWV), to deliver which one furnishes the best results in analysis terms of this type of biomedical signals.

The signals that will be the subject of this comparative study are extracted from [19]. These signals are chosen with different pathologies and variant forms in order to make the study more credible.

This paper is organized as follows: the "Theoretical background" section is dedicated to present the chosen denoising methods, the three time-frequency techniques, and the selected ECG signals. For a qualitative performance, comparison of the denoising methods and the time-frequency techniques is performed in the "Results and discussion" section, accompanied with a discussion of the obtained results. Finally, this study is concluded with a "Conclusion" section. 


\section{Theory background}

\subsection{Denoising techniques used}

\subsubsection{Empirical mode decomposition (EMD)}

Huang et al. had defined a tool named EMD to decompose adaptively a signal in a set of AM-FM components [5]. No mathematical foundations or analytical expressions have been proposed for the technique theoretical study. In various domains, such as biomedicine, acoustics, seismology, or study of climate phenomena, the EMD has been used successfully in several works to treat real data [20,21]. These studies had provided satisfaction and good results in signal processing, especially for nonstationary ones. A nonstationary signal is decomposed adaptively by the EMD technique into a sum of functions oscillatory band-limited $d(t)$. These functions, called intrinsic mode functions $\operatorname{IMF}_{J}(t)$, oscillate around zero. The intrinsic mode functions can express the signal $\mathrm{x}(\mathrm{t})$ by the following expression:

$$
x(t)=\sum_{j=1}^{k} d_{j}(t)+r(t)
$$

where $r(t)$ is the low-frequency residue.

Two conditions must be satisfied by each $\operatorname{IMF}_{\mathrm{J}}(\mathrm{t})$ :

- The zero crossings and extreme signal numbers must be equal all over the analyzed signal.

- The envelope average defined by signal local extreme must be equal to 0 at any point. On the one hand, the low-oscillation components are represented by the higher-order $\operatorname{IMF}_{\mathrm{J}}(\mathrm{t})$, and on the other hand, the fast ones are presented by lower-order $\operatorname{IMF}_{\mathrm{J}}(\mathrm{t})$. The $\operatorname{IMF}_{\mathrm{J}}(\mathrm{t})$ number is variable for different decomposed signals and depends on the signal spectral content. The technical aspects of the EMD implementation are decomposed on five steps given by the following algorithm [5]:

Step 1: Extraction of the signal $\mathrm{x}(\mathrm{t})$ extreme.

Step 2: By the maximum interpolation (resp. minima), an upper envelope $\mathrm{e}_{\max }(\mathrm{t})$ (resp. lower $\mathrm{e}_{\min }(\mathrm{t})$ ) is deduced.

Step 3: The half envelope sum is defined as a local average $\mathrm{m}(\mathrm{t})$ by the following expression:

$$
m(t)=\left(e_{\max }(t)+e_{\min }(t)\right) / 2
$$

Step 4: Deduction of $d_{J}(t)=\operatorname{IMF}_{J}(t)$, a local detail by

$$
d(t)=x(t)-m(t)
$$

Step 5: The expression (1) gives the iteration.

The high frequency terms are contained in the first IMF, which also involves the following terms of decreasing frequency up to forwarding only a low-frequency residue. 


\subsubsection{Ensemble empirical mode decomposition (EEMD)}

The ensemble empirical mode decomposition (EEMD) method was proposed to surpass the mode mixing disadvantage which exists in EMD technique [22]. By repeating the processes of decomposition, the EMD provides all solutions giving the true IMF.

The following steps give the EEMD method algorithm:

Step 1: The analyzed signal is added with a predefined amplitude white noise.

Step 2: The resulted signal is decomposed by using the EMD method.

Step 3: The above signal decomposition is repeated with different fixed amplitude white noises.

Step 4: Calculation of the final results is equal to the ensemble means of the decomposition results.

As finite number of intrinsic mode functions (IMFs) and a residue, the signal $\mathrm{x}$ (k) is decomposed:

$$
x(k)=\sum_{i=1}^{n} \widehat{c}+\widehat{r}
$$

where $\mathrm{n}$ defines the IMF number, $\widehat{c}_{i}$ is the $\mathrm{i}$-th IMF which is the corresponding IMF ensemble mean resulted from all of the decomposition processes, and $\widehat{r}$ is the residue mean obtained from all processes of the decomposition.

\subsubsection{Complete ensemble empirical mode decomposition with adaptive noise (CEEMDAN)}

\subsubsection{CEEMD algorithm}

Although the mode mixing effect is mitigated by the EEMD method, if the ensemble number is small, some noise will continue to exist in the corresponding $\operatorname{IMF}(\mathrm{s})$. To deal with this problem and assure a noise-free IMF, a CEEMD algorithm [7-23] is defined by the following steps:

Step 1: The target signal $\mathrm{x}(\mathrm{t})$ is added by positive and negative white noise $\varepsilon^{+},-(t)$ in order to create two new signals $x^{+}(t)$ and $x^{-}(t)$ :

$$
\begin{aligned}
& x^{+}(t)=x(t)+\varepsilon^{+}(t) \\
& x^{-}(t)=x(t)+\varepsilon^{-}(t)
\end{aligned}
$$

Step 2: Step 1 is repeated, and by using the EMD algorithm, each of the new signals $\mathrm{x}^{+}(\mathrm{t})$ and $\mathrm{x}^{-}(\mathrm{t})$ is decomposed.

Step 3: For the $\mathrm{x}^{+}(\mathrm{t})$ and $\mathrm{x}^{-}(\mathrm{t})$ data sets, two IMF sets are obtained; (4) by averaging the $I M F_{k}^{i}$ in Eq. (11), the decomposed result is calculated, where $I M F_{k}^{i}$ defines the i-th IMF of the k-th iteration;

$$
\overline{I M F}_{1}(n)=\frac{1}{I} \sum_{i=1}^{l} \overline{I M F_{k}^{i}}(n)
$$

\subsubsection{Complete ensemble empirical mode of decomposition with adaptive noise (CEEMDAN)}

On the one hand, using EEMD overcomes the EMD mode mixing problem, but on the other hand, this technique presents a problem. The number of iterations 
required in EEMD process is higher, added to the residual noise remaining in the reconstructed signal. So we have a new method called CEEMD; this technique provides an exact reconstruction of the original signal and gives better separation of modes with low computation cost. In this method the first mode $\overline{\operatorname{IMF}}_{1}(\mathrm{n})$ is obtained in the same way as in EEMD. It's computed over an ensemble of $r_{1}(n)$ plus different realizations of a given noise obtaining $\overline{\mathrm{IMF}}_{2}(\mathrm{n})$ by averaging. Here $E_{j}[$.$] operator provides j^{j^{t h}}$ mode obtained by EMD. $w^{i}$ is the white noise [7-24].

The steps of CEEMDAN decomposition are as follows:

Step 1: Decompose $\mathrm{x}(\mathrm{n})+\varepsilon_{0} \mathrm{w}_{\mathrm{i}}(\mathrm{n})$ to obtain the first mode by using:

$$
\overline{\operatorname{IMF}}_{1}(n)=\frac{1}{I} \sum_{i=1}^{l} \overline{\operatorname{IMF} F_{k}^{i}}(n)
$$

where $w_{0}$ is the added white noise amplitude and $\varepsilon(t)$ is the white noise with unit variance.

Step 2: Compute the difference signal:

$$
r_{1}(n)=x(n)-\overline{I M F}_{1}(n)
$$

Step 3: Decompose $r_{1}(n)+w_{1} E_{1}\left(\varepsilon^{i}(n)\right)$, to obtain the first mode, and define the second mode by

$$
\overline{I M F}_{2}(n)=\frac{1}{I} \sum_{i=1}^{l} E_{1}\left(r_{1}(n)+w_{1} E_{1}\left(\varepsilon^{i}(n)\right)\right)
$$

For $\mathrm{k}=2, \ldots, \mathrm{K}$, calculate the $\mathrm{k}$-th residue and obtain the first mode. Define the $(k+1)$-th mode as follows:

$$
\overline{I M F}_{k+1}(n)=\frac{1}{I} \sum_{i=1}^{l} E_{1}\left(r_{k}(n)+w_{k} E_{k}\left(\varepsilon^{i}(n)\right)\right)
$$

where $E_{1}$ is a function to extract the $\mathrm{j}^{\text {th }}$ IMF decomposed by EMD.

Step 4: Continue this process until residue is no longer feasible. Final residue

$$
R(n)=x(n)-\sum_{k=1}^{k} \overline{I M F}_{k}
$$

So the given signal can be expressed as

$$
x(n)=R(n)+\sum_{k=1}^{k} \overline{I M F}_{k}
$$

A quantitatively comparison of these three filtering methods' performance will be made based on two metrics: mean square error (MSE) and percent root mean square difference (PRD). The MSE and PRD are used to evaluate the quality of the information which is preserved in the denoised ECG signal. The MSE and the PRD are computed as follows:

$$
M S E=\frac{1}{N} \sum_{n=1}^{N}(x(n)-\bar{x}(n))^{2}
$$




$$
P R D=\sqrt{\frac{\sum_{n=1}^{N}(x(n)-\bar{x}(n))^{2}}{\sum_{n=1}^{N} x^{2}(n)}} * 100
$$

where $\mathrm{x}(\mathrm{n})$ is the original ECG signal, $\bar{x}(n)$ denotes the reconstruction of the ECG signal, and $\mathrm{N}$ is the number of ECG samples used.

\subsection{Time-frequency techniques}

In time-varying spectral analysis, time-frequency techniques have found a large number of application [8-15]. There is no single time-frequency representation due to the signal energy distribution which joints time and frequency coordinates. There are many time-frequency techniques and many ways to define them. The most popular time-frequency representation class is called the quadratic or Cohen (1989) class. The Choi-Williams (CW), the periodgram (PE), and the smoothed pseudo Wigner-Ville (SPWV) techniques were chosen from the different time-frequency techniques belonging to this representation time-frequency set, due to its interesting properties.

\subsubsection{Choi-Williams distribution (CWD)}

The Choi-Williams distribution $\operatorname{CWD}(t, f)$ was a significant step in the field of time-frequency analysis where it opened the way for optimizing resolution with cross-term reduction [25]:

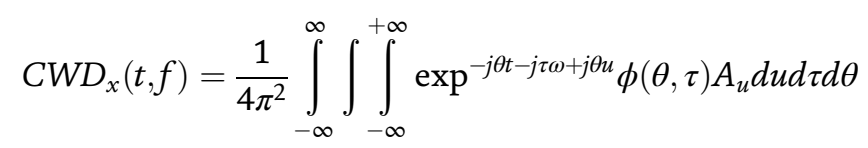

where

$$
A_{u}=x\left(u+\frac{\tau}{2}\right) x^{*}\left(u-\frac{\tau}{2}\right)
$$

and $\phi(\theta, \tau)=e^{\frac{\theta^{2} \tau^{2}}{\sigma}}$.

The smoothing of the distribution is controlled by the constant $\sigma$. If $\sigma \rightarrow \infty$, the Choi-Williams distribution (CWD) will simply converge to the Wigner-Ville distribution, as the kernel goes to 1 .

\subsubsection{Periodogram technique}

The minimum variance estimator, named Capon estimator (CA), does not fix a model on the signal. At each frequency $f$, this method seeks a matched filter whose response is 1 for the frequency $f$ and 0 everywhere else [26]:

$$
C A(t, f)=a(n, f)^{H} R_{x} a(t, f)=\frac{1}{Z_{f}^{H} \cdot R_{x}[t]^{-1} \cdot Z_{f}}
$$

where $\mathrm{CA}(\mathrm{n}, \mathrm{f})$ means the filter Capon output power. By the discrete signal $\mathrm{x}(\mathrm{n})$ sampled at the period $t_{e}$, this filter is excited; $a(n, f)=\left(a_{0}, \ldots, a_{p}\right)$ is the filter impulse response at frequency $n ; R_{x}[n]=E\left\{x[n] x^{T}[n]\right\}$ is the crossed $x(n)$ autocorrelation matrix of dimension $(\mathrm{p}+1)^{*}(\mathrm{p}+1) ; \mathrm{x}[\mathrm{n}]=(\mathrm{x}(\mathrm{n}-\mathrm{p}), \ldots, \mathrm{x}(\mathrm{n}))$ is the selected signal at time $n ; Z_{f}^{H}\left(1, e_{e}^{2 i \pi f t}, \ldots, e_{e}^{2 i \pi f t} p\right)$ is the steering vector; $(p+1)$ is 
the number of filter coefficient and the exponent $\mathrm{H}$ for conjugate transpose and the superscript $\mathrm{T}$ for transpose.

The periodogram (PE) is the derivate of the Capon (CA) technique. The spectral estimator of this method is defined by the following equation [26]:

$$
P E(t, f)=Z_{f}^{H} \cdot R_{x} \cdot Z_{f} /\left((\mathrm{p}+1)^{2}\right)
$$

By sliding windows, the PE technique can be used. Theoretical criterion does not exist for selecting window duration and filter order. The parametric technique frequency response presents different properties according to the signal characteristics. The time-frequency resolution depends principally of the window choice. Usually, the PE estimator gives a better frequency resolution.

\subsubsection{Smoothed pseudo Wigner-Ville technique (SPWV)}

The Cohen class exhibits most nonparametric time-frequency techniques $[16,17]$. The smoothed pseudo Wigner-Ville technique belongs in particular to this class $[16,17]$. To overcome the major weakness of the Wigner-Ville time-frequency representation, which is the covering of frequential components, the SPWV has been proposed between the different existing nonparametric time-frequency techniques; for that, the analytical signal $\mathrm{x}_{\mathrm{a}}(\mathrm{t})$ replaces the real signal $\mathrm{x}(\mathrm{t})$. The following expression defines this signal:

$$
x_{a}(t)=x(t)+i H\{x(t)\}
$$

where $\mathrm{i}^{2}=-1, \mathrm{H}\{\mathrm{x}(\mathrm{t})\}$ is the Hilbert transform of the signal with real values, $\mathrm{x}(\mathrm{t})$.

Expression (20) defines the analytical signal $\mathrm{x}_{\mathrm{a}}(\mathrm{t})$ spectrum, $\mathrm{F}_{\mathrm{a}}(\mathrm{k})$ :

$$
F_{a}(k)= \begin{cases}2 X(k) & \text { if } 0<k<N / 2 \\ X(0) & \text { if } k=0, N / 2 \\ 0 & \text { if } N / 2<k<N\end{cases}
$$

where $\mathrm{X}(\mathrm{k})$ represents the original signal $\mathrm{x}(\mathrm{t})$ Fourier transform and $\mathrm{N}$ is the point number.

The function $\mathrm{W}_{\mathrm{x}}(t, \mathrm{f})$ is the Wigner-Ville distribution related to a signal $\mathrm{x}(\mathrm{t})$, of finished energy. This distribution depends on the temporal $(t)$ and frequential $(f)$ parameters. The following expression defines this distribution $[16,17]$ :

$$
W_{x_{a}}(t, f)=\int_{-\infty}^{+\infty} x_{a}\left(t+\frac{\tau}{2}\right) \cdot x^{*}{ }_{a}\left(t-\frac{\tau}{2}\right) e^{-2 i n f \tau} d \tau
$$

where $\mathrm{x}_{\mathrm{a}}{ }_{\mathrm{a}}(\mathrm{t})$ indicates the complex conjugate of $\mathrm{x}_{\mathrm{a}}(\mathrm{t})$.

The SPWV is used principally to decrease the problem of the interference terms happening between the inner components that existed in Wigner-Ville image. The time-frequency image visibility is reduced by these terms $[13,14]$. The SPWV technique is applied by using two smoothing windows $\mathrm{h}(\mathrm{t})$ and $\mathrm{g}(\mathrm{t})$. The utility of these smoothing windows entered into the definition of the Wigner-Ville technique is to guarantee an interference separate control both in time $(\mathrm{g})$ and in frequency $(\mathrm{h})$. This representation is defined by the following expression $[16,17]$ : 


$$
\begin{aligned}
S P W V_{x}(t, f)= & \int_{-\infty}^{+\infty} h\left|\left(\frac{\tau}{2}\right)^{2}\right| \int_{-\infty}^{+\infty} g(t-u) x_{a}\left(u+\frac{\tau}{2}\right) \\
& x_{a}^{*}\left(u-\frac{\tau}{2}\right) e^{-2 i \pi f \tau} d \tau d u
\end{aligned}
$$

where $\mathrm{h}(\mathrm{t})$ is a smoothing frequential window and $\mathrm{g}(\mathrm{t})$ is a smoothing temporal window.

We compare also the performance of these three time-frequency techniques by using the same metrics that were used in the filtering method comparison.

\subsection{Biomedical signals}

Electrocardiogram signals allow to represent the human heart state. ECG signal is a fundamental tool commonly used in the heart medical domain to treat patients suffering from cardiac diseases. By measuring the potential difference between electrodes posed in well-known places in the patient skin, these signals are usually obtained. The ECG signal can be single channel or multichannel depending on how many electrodes are used, one or several. Important knowledge is obtained by cardiologists about the patient's heart function only by analyzing a minute feature of these signals.

The ECG signal has a well-defined P, QRS, and T signatures that represent each heartbeat. The duration, shape, and amplitude of these waves are considered as major features in time domain analysis.

Changes in the normal rhythmicity of a human heart may result in different cardiac arrhythmias, which may be immediately fatal or cause irreparable damage to the heart when sustained over a long period of time.

The following subsections present the different normal and abnormal ECG signals chosen for this study. These data were obtained from [19].

\subsubsection{Normal ECG}

Figure 1 shows the time domain of a normal ECG signal. The sampling frequency for this normal ECG signal was 128 samples/s and the signal length $8 \mathrm{~s}$.

\subsubsection{Atrial fibrillation ECG}

Figure 2 shows a length of $4 \mathrm{~s}$ of an abnormal atrial fibrillation ECG signal obtained from a patient with malignant ventricular arrhythmia. The sampling frequency for this signal was 250 samples/s.

The atrial rate exceeds 350 beats per minute in this type of arrhythmias. This arrhythmia occurs due to an uncoordinated activation and contraction of different parts of the atrial which leads to ineffective pumping of blood into the ventricles.

\subsubsection{Ventricular tachyarrhythmia ECG}

Figure 3 shows a length of $4 \mathrm{~s}$ of a ventricular tachyarrhythmia ECG signal with a 250 samples/s sampling frequency.

This abnormal signal presents a misalignment of the third QRS complex. 
Combination of the CEEM Decomposition with Adaptive Noise and Periodogram Technique... DOI: http://dx.doi.org/10.5772/intechopen.86007

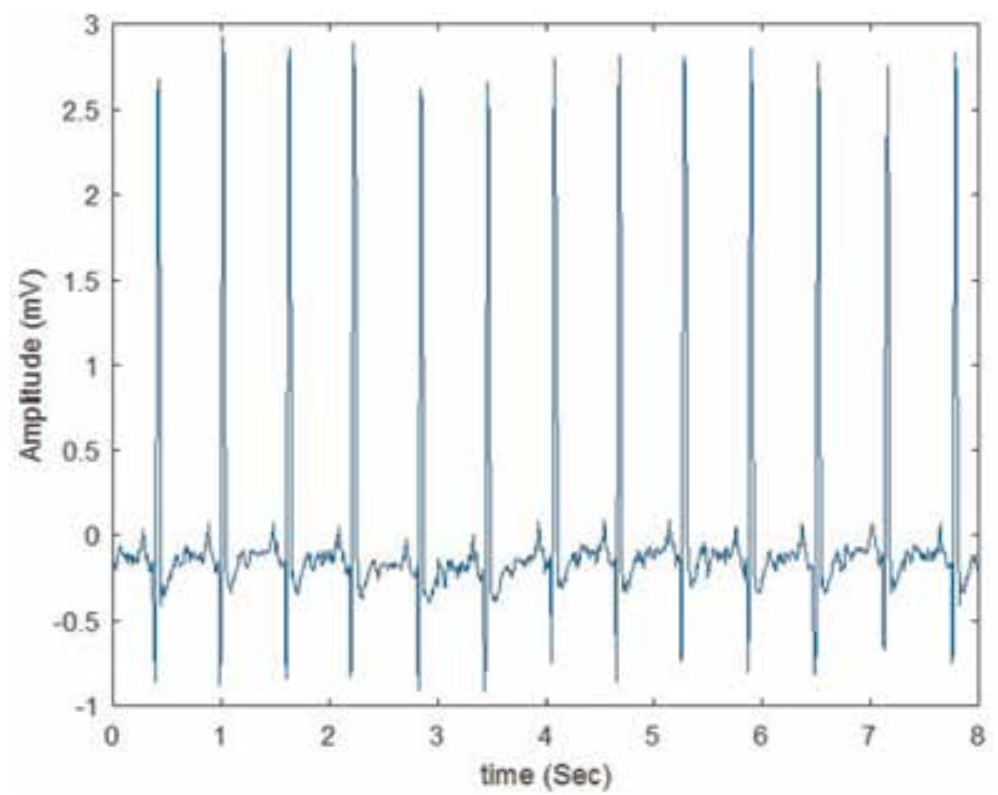

Figure 1.

Normal ECG signal.

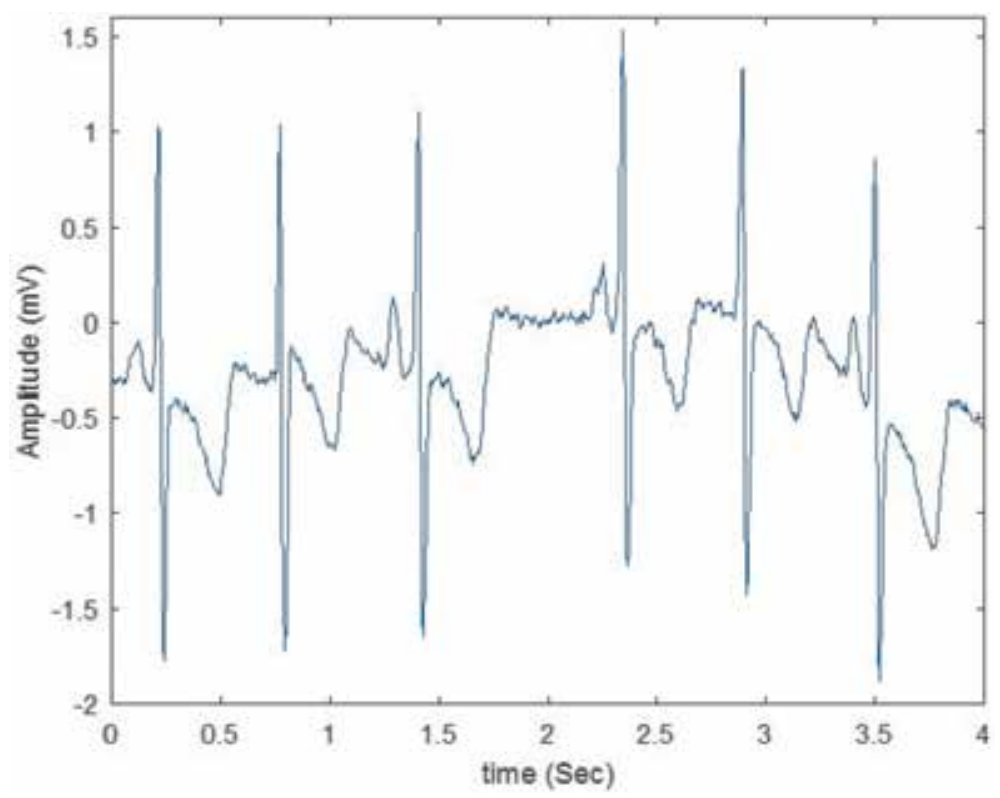

Figure 2.

Atrial fibrillation ECG signal.

\subsubsection{Malignant ventricular arrhythmia ECG}

Figure 4 shows a length of $4 \mathrm{~s}$ of the time domain ECG signal obtained from a patient with malignant ventricular arrhythmia. The sampling frequency for this signal was 250 samples/s. The depolarization wave spreads through the ventricles by an irregular and therefore slower pathway. The QRS complex is thus wide and abnormal. Repolarization pathways are also different, causing the $\mathrm{T}$ wave to have an unusual morphology. 


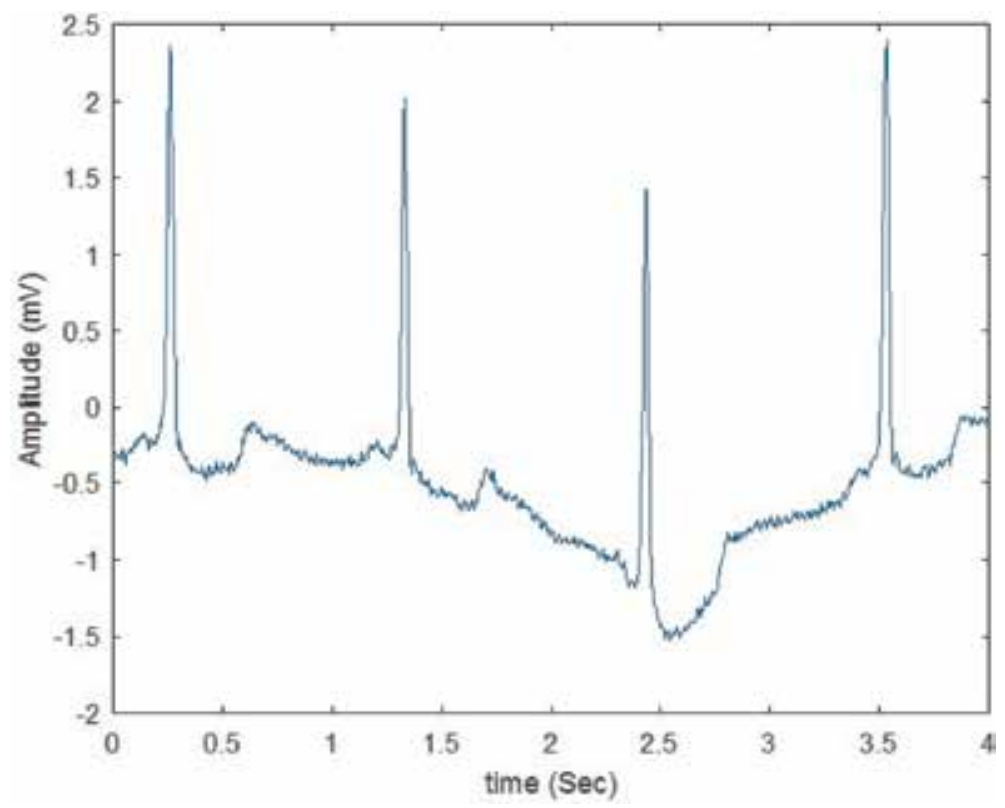

Figure 3.

Ventricular tachyarrhythmia ECG signal.

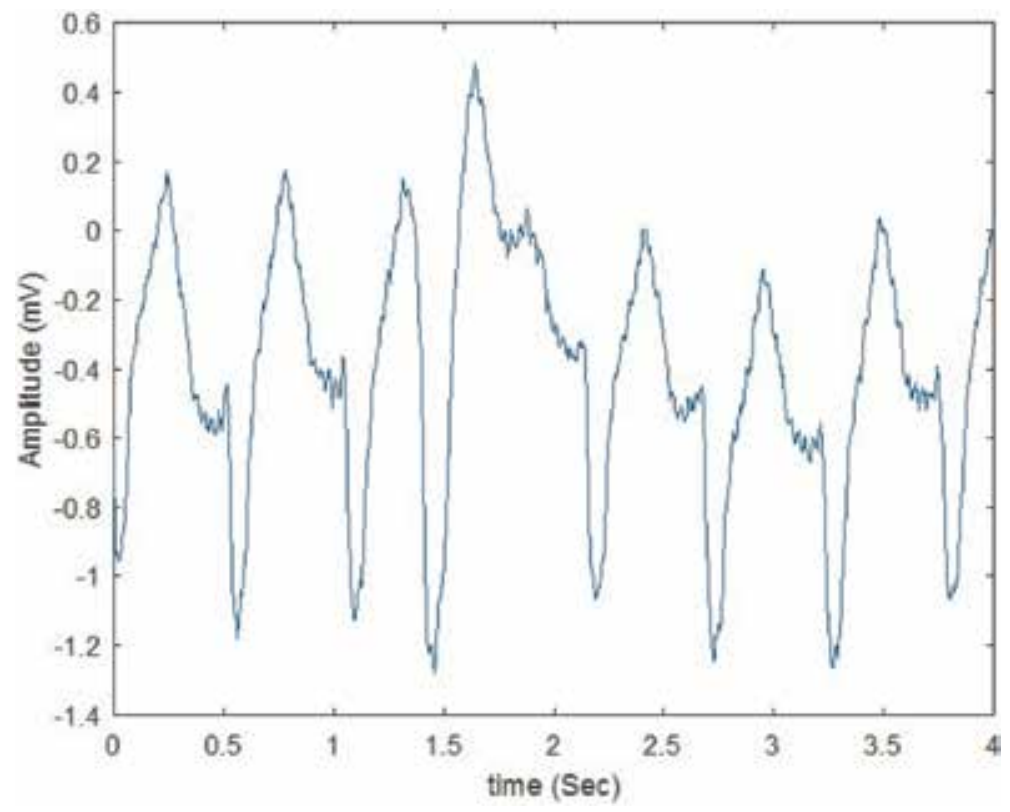

Figure 4.

Malignant ventricular arrhythmia ECG signal.

\subsubsection{Supraventricular ECG}

Figure 5 shows the time domain ECG signal of a patient with supraventricular arrhythmia. The sampling frequency for this abnormal ECG signal was 128 samples/ $\mathrm{s}$ and the signal length $8 \mathrm{~s}$. The shape of the QRS complex in this signal is abnormal at the QR part. 


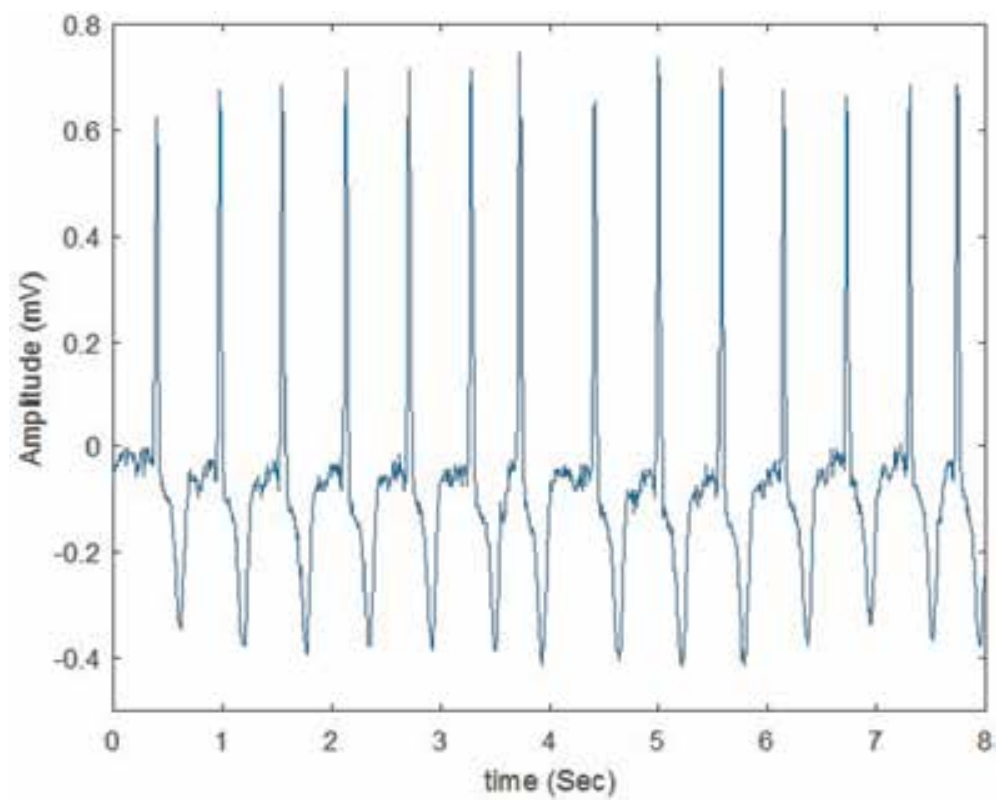

Figure 5 .

Supraventricular arrhythmia ECG signal.

These normal and abnormal ECG signals were corrupted with noise CN generated by the following Eq. [4]:

$$
C N=\frac{w b w * B W+w e m * E M+w m a * M A}{w b w+w e m+w m a}
$$

where $\mathrm{BW}$ is the baseline wander noise, EM is the electromyogram noise, and MA is the motion artifact. Wbw, wem, and wma define the added noise percentage of baseline wander, electromyogram noise, and motion artifact noises, respectively. These parameters have been chosen with the following values wbw $=2$, wem $=2$, and wma $=5$, which signified that the predominant noise in the noisy ECG signal is the motion artifact.

\section{Results and discussion}

\subsection{Denoising methods}

To evaluate the performance of the three denoising methods, EMD, EEMD, and CEEMDAN, a set of normal and abnormal ECG signals with different shapes were chosen. Before applying the proposed denoising methods, the ECG signals were corrupted with different values of signal-to-noise ratio (SNR); these values are from $-5 \mathrm{~dB}$ to $20 \mathrm{~dB}$ with a $5 \mathrm{~dB}$ step.

Tables 1-5 report the performance of the denoising methods for the five ECG recordings. These tables present the obtained results of the mean square error (MSE), the root mean square error (RMSE), and the percent root mean square difference (PRD) for the following ECG signals, respectively, a normal ECG, an atrial fibrillation ECG, a ventricular tachyarrhythmia ECG, a malignant ventricular arrhythmia ECG, and a supraventricular arrhythmia ECG.

Figures 6-10 present the RMSE comparison graphs of the results obtained by using different denoising methods (EMD, EEMD, and CEEMDAN) to the five 


\begin{tabular}{cccccccccc}
\hline \multirow{2}{*}{ SNR } & \multicolumn{3}{c}{ EMD } & \multicolumn{3}{c}{ EEMD } & \multicolumn{3}{c}{ CEEMDAN } \\
\cline { 2 - 9 } & MSE & RMSE & PRD & MSE & RMSE & PRD & MSE & RMSE & PRD \\
\hline-5 & 1.179 & 1.086 & 192.033 & 1.011 & 1.006 & 177.847 & 1.047 & 1.023 & 180.914 \\
\hline 0 & 0.450 & 0.671 & 118.580 & 0.320 & 0.565 & 99.966 & 0.357 & 0.597 & 105.620 \\
\hline 5 & 0.227 & 0.476 & 84.191 & 0.101 & 0.318 & 56.303 & 0.136 & 0.368 & 65.094 \\
\hline 10 & 0.136 & 0.368 & 65.104 & 0.032 & 0.180 & 31.820 & 0.064 & 0.253 & 44.812 \\
\hline 15 & 0.055 & 0.235 & 41.628 & 0.010 & 0.101 & 17.900 & 0.042 & 0.205 & 36.252 \\
\hline 20 & 0.038 & 0.196 & 34.604 & 0.003 & 0.059 & 10.359 & 0.034 & 0.185 & 32.695 \\
\hline
\end{tabular}

Table 1.

MSE, RMSE, and PRD of the normal ECG signal.

\begin{tabular}{cccccccccc}
\hline \multirow{2}{*}{ SNR } & \multicolumn{3}{c}{ EMD } & \multicolumn{3}{c}{ EEMD } & \multicolumn{3}{c}{ CEEMDAN } \\
\cline { 2 - 9 } & MSE & RMSE & PRD & MSE & RMSE & PRD & MSE & RMSE & PRD \\
\hline-5 & 0.780 & 0.883 & 179.314 & 0.768 & 0.877 & 177.928 & 0.767 & 0.876 & 177.727 \\
\hline 0 & 0.254 & 0.504 & 102.241 & 0.243 & 0.493 & 100.025 & 0.243 & 0.493 & 100.150 \\
\hline 5 & 0.097 & 0.311 & 63.129 & 0.077 & 0.277 & 56.300 & 0.078 & 0.280 & 56.837 \\
\hline 10 & 0.042 & 0.204 & 41.404 & 0.025 & 0.157 & 31.797 & 0.026 & 0.163 & 33.003 \\
\hline 15 & 0.023 & 0.153 & 31.050 & 0.008 & 0.087 & 17.760 & 0.010 & 0.099 & 20.117 \\
\hline 20 & 0.017 & 0.132 & 26.796 & 0.003 & 0.051 & 10.373 & 0.005 & 0.070 & 14.131 \\
\hline
\end{tabular}

Table 2.

MSE, RMSE, and PRD of the atrial fibrillation ECG signal.

\begin{tabular}{cccccccccc}
\hline \multirow{2}{*}{ SNR } & \multicolumn{3}{c}{ EMD } & \multicolumn{3}{c}{ EEMD } & \multicolumn{3}{c}{ CEEMDAN } \\
\cline { 2 - 9 } & MSE & RMSE & PRD & MSE & RMSE & PRD & MSE & RMSE & PRD \\
\hline-5 & 1.590 & 1.261 & 177.669 & 1.590 & 1.261 & 177.694 & 1.589 & 1.261 & 177.640 \\
\hline 0 & 0.513 & 0.716 & 100.957 & 0.505 & 0.711 & 100.173 & 0.503 & 0.709 & 99.905 \\
\hline 5 & 0.173 & 0.416 & 58.550 & 0.159 & 0.399 & 56.224 & 0.159 & 0.399 & 56.195 \\
\hline 10 & 0.057 & 0.240 & 33.782 & 0.051 & 0.225 & 31.769 & 0.050 & 0.224 & 31.618 \\
\hline 15 & 0.029 & 0.169 & 23.884 & 0.016 & 0.127 & 17.906 & 0.016 & 0.127 & 17.863 \\
\hline 20 & 0.016 & 0.126 & 17.685 & 0.005 & 0.074 & 10.403 & 0.005 & 0.072 & 10.151 \\
\hline
\end{tabular}

Table 3 .

MSE, RMSE, and PRD of the ventricular tachyarrhythmia ECG signal.

considered ECG signals: normal ECG, atrial fibrillation ECG, ventricular tachyarrhythmia ECG, malignant ventricular arrhythmia ECG, and supraventricular arrhythmia ECG, respectively, at a SNR interval varying from -5 to $20 \mathrm{~dB}$.

Figures 11-15 are presenting the obtained PRD results of the three denoising methods (EMD, EEMD, and CEEMDAN) to all the chosen ECG signals, normal ECG, atrial fibrillation ECG, ventricular tachyarrhythmia ECG, malignant ventricular arrhythmia ECG, and supraventricular arrhythmia ECG, respectively, at a SNR interval varying from -5 to $20 \mathrm{~dB}$. 
Combination of the CEEM Decomposition with Adaptive Noise and Periodogram Technique... DOI: http://dx.doi.org/10.5772/intechopen.86007

\subsection{Time-frequency techniques}

To compare the performance of the three chosen time-frequency techniques, Choi-Williams (CW), periodogram (PE), and smoothed pseudo Wigner-Ville (SPWV), we applied these time-frequency methods to ECG signals presented in

\begin{tabular}{lccccccccc}
\hline \multirow{2}{*}{ SNR } & \multicolumn{3}{c}{ EMD } & \multicolumn{3}{c}{ EEMD } & \multicolumn{3}{c}{ CEEMDAN } \\
\cline { 2 - 9 } & MSE & RMSE & PRD & MSE & RMSE & PRD & MSE & RMSE & PRD \\
\hline-5 & 0.918 & 0.958 & 177.481 & 0.922 & 0.960 & 177.902 & 0.920 & 0.959 & 177.673 \\
\hline 0 & 0.291 & 0.539 & 99.900 & 0.291 & 0.540 & 99.946 & 0.291 & 0.539 & 99.921 \\
\hline 5 & 0.092 & 0.303 & 56.142 & 0.092 & 0.304 & 56.221 & 0.092 & 0.303 & 56.214 \\
\hline 10 & 0.029 & 0.171 & 31.672 & 0.029 & 0.171 & 31.692 & 0.029 & 0.171 & 31.628 \\
\hline 15 & 0.009 & 0.096 & 17.863 & 0.009 & 0.096 & 17.856 & 0.009 & 0.096 & 17.846 \\
\hline 20 & 0.003 & 0.056 & 10.291 & 0.003 & 0.055 & 10.214 & 0.003 & 0.055 & 10.122 \\
\hline
\end{tabular}

Table 4.

MSE, RMSE, and PRD of the malignant ventricular arrhythmia ECG signal.

\begin{tabular}{cccccccccc}
\hline \multirow{2}{*}{ SNR } & \multicolumn{3}{c}{ EMD } & \multicolumn{3}{c}{ EEMD } & \multicolumn{3}{c}{ CEEMDAN } \\
\cline { 2 - 9 } & MSE & RMSE & PRD & MSE & RMSE & PRD & MSE & RMSE & PRD \\
\hline-5 & 0.137 & 0.370 & 179.290 & 0.135 & 0.367 & 177.814 & 0.135 & 0.367 & 177.867 \\
\hline 0 & 0.047 & 0.217 & 105.004 & 0.043 & 0.206 & 99.982 & 0.043 & 0.207 & 100.557 \\
\hline 5 & 0.017 & 0.131 & 63.466 & 0.013 & 0.116 & 56.276 & 0.014 & 0.119 & 57.717 \\
\hline 10 & 0.008 & 0.090 & 43.633 & 0.004 & 0.066 & 31.748 & 0.005 & 0.071 & 34.536 \\
\hline 15 & 0.006 & 0.079 & 38.091 & 0.001 & 0.037 & 18.000 & 0.002 & 0.047 & 22.774 \\
\hline 20 & 0.004 & 0.061 & 29.538 & 0.0005 & 0.021 & 10.400 & 0.001 & 0.037 & 17.705 \\
\hline
\end{tabular}

Table 5.

MSE, RMSE, and PRD of the supraventricular arrhythmia ECG signal.

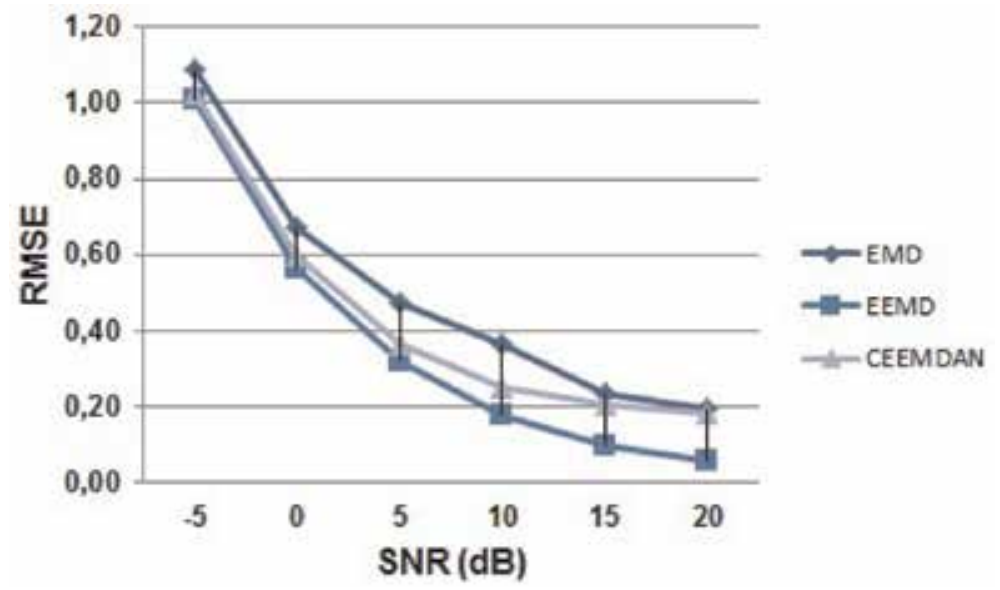

Figure 6.

RMSE comparison of the three denoising methods at different SNR levels for the normal ECG signal. 


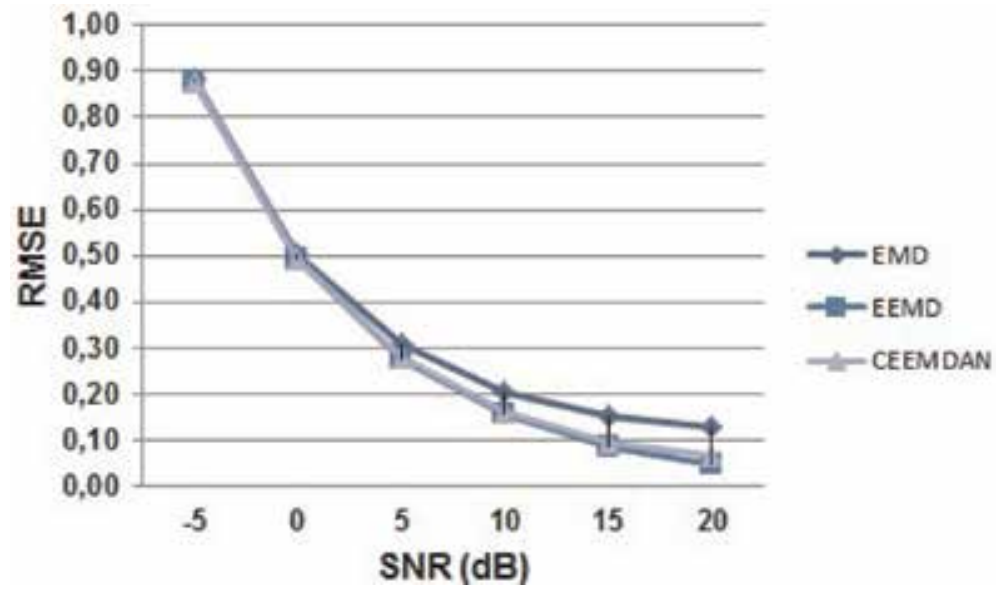

Figure 7.

RMSE comparison of the three denoising methods at different SNR levels for the atrial fibrillation ECG signal.

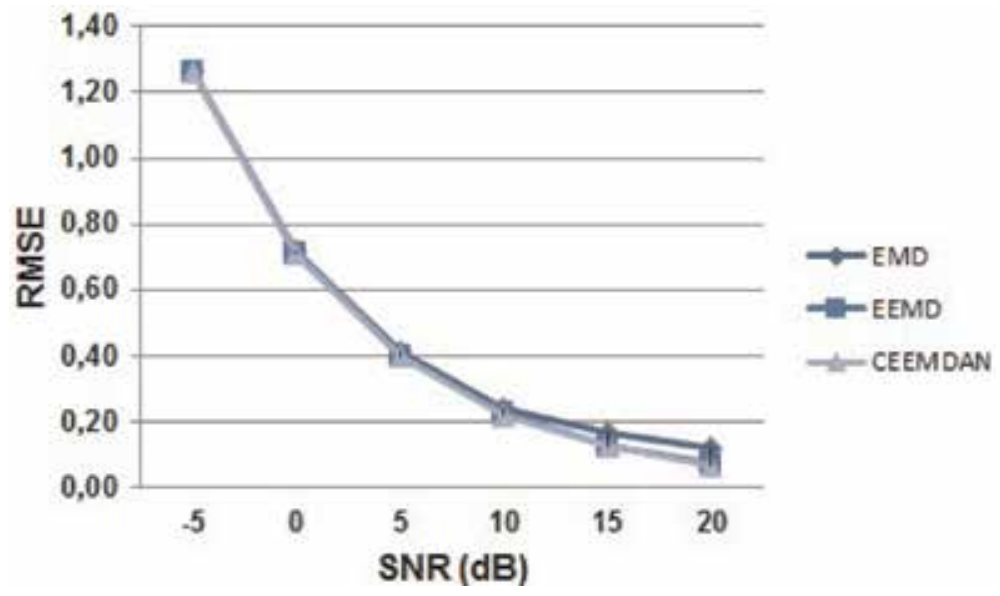

Figure 8.

RMSE comparison of the three denoising methods at different SNR levels for the ventricular tachyarrhythmia ECG signal.

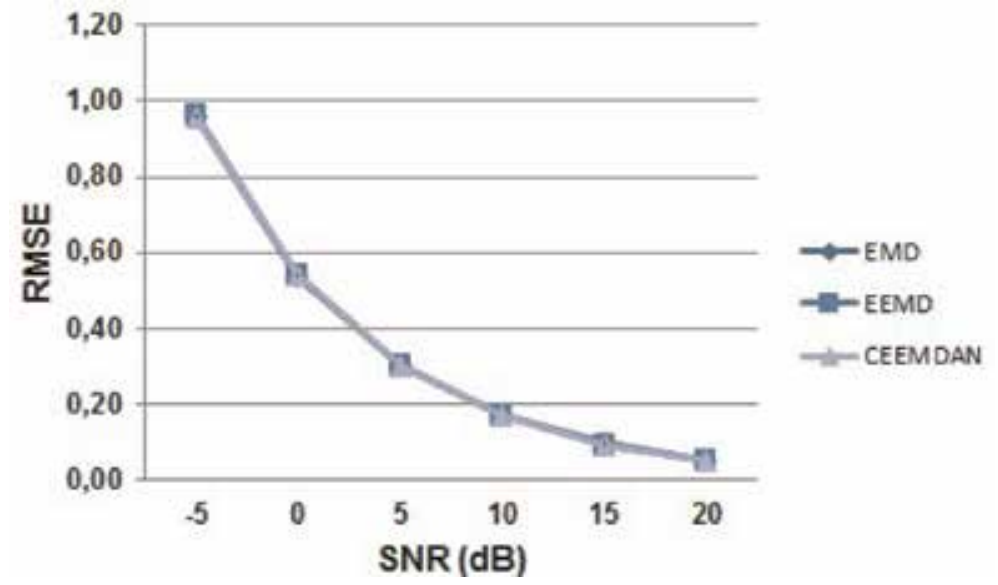

Figure 9.

RMSE comparison of the three denoising methods at different SNR levels for the malignant ventricular arrhythmia ECG signal. 
Combination of the CEEM Decomposition with Adaptive Noise and Periodogram Technique... DOI: http://dx.doi.org/10.5772/intechopen.86007

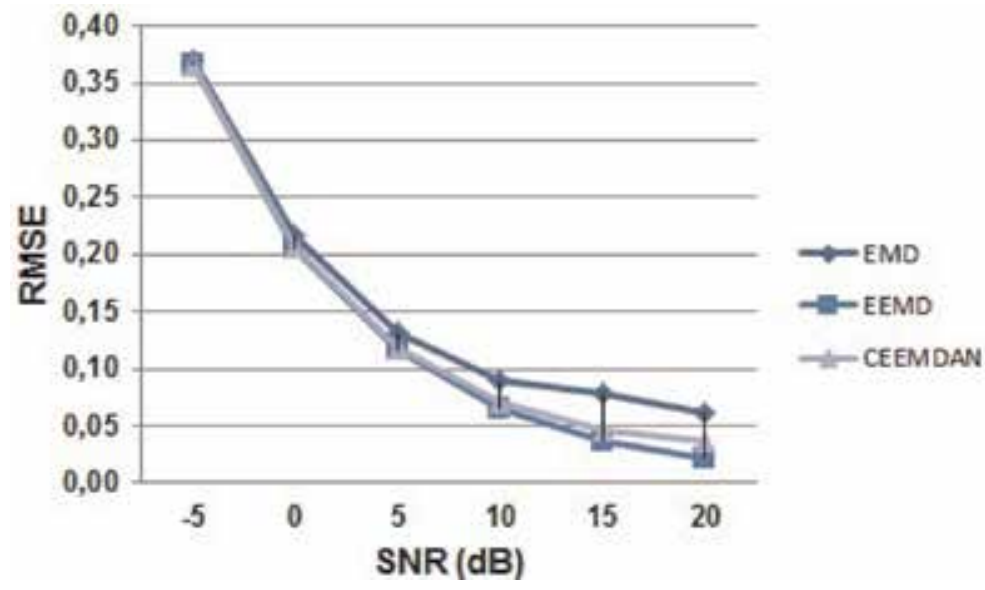

Figure 10.

RMSE comparison of the three denoising methods at different SNR levels for the supraventricular arrhythmia ECG signal.

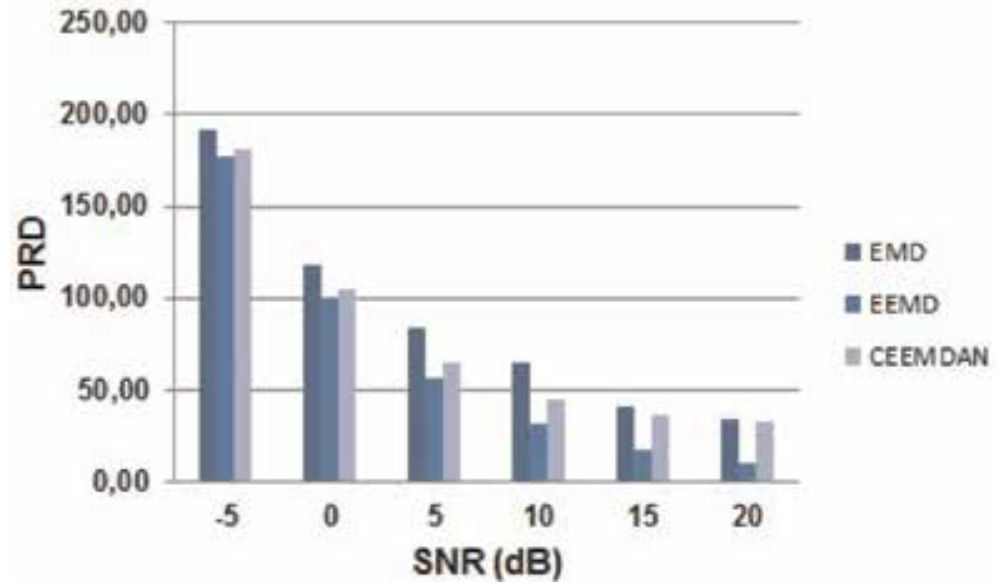

Figure 11.

PRD comparison of the different denoising methods at different SNR levels for the normal ECG signal.

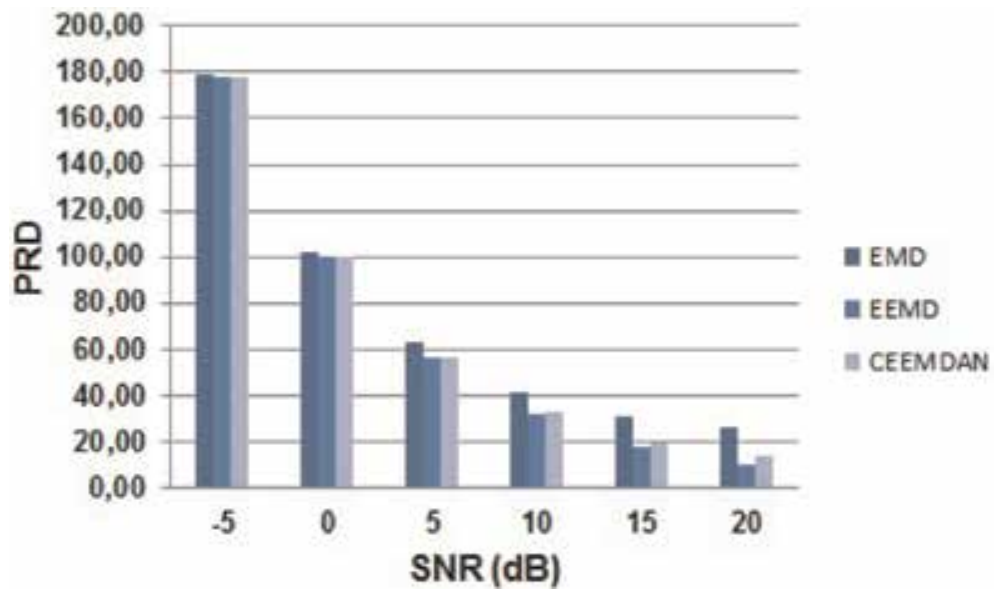

Figure 12.

PRD comparison of the different denoising methods at different SNR levels for the atrial fibrillation ECG signal. 


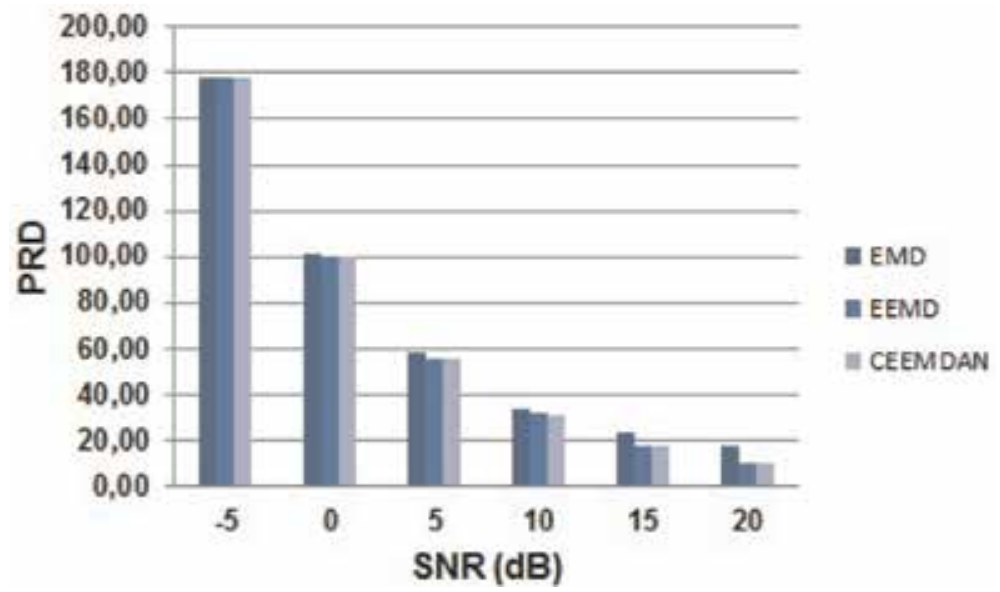

Figure 13.

PRD comparison of the different denoising methods at different SNR levels for the ventricular tachyarrhythmia ECG signal.

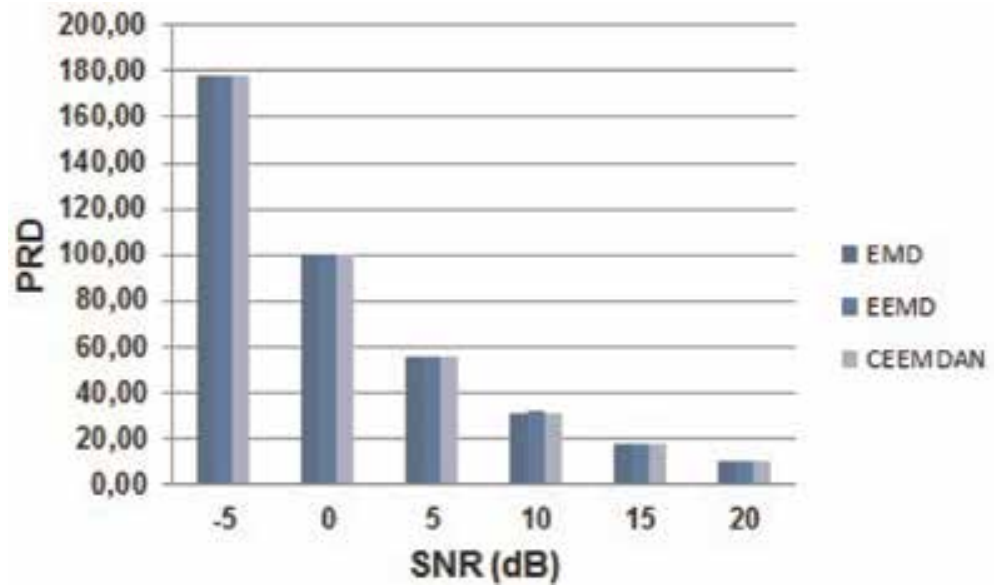

Figure 14.

PRD comparison of the different denoising methods at different SNR levels for the malignant ventricular arrhythmia ECG signal.

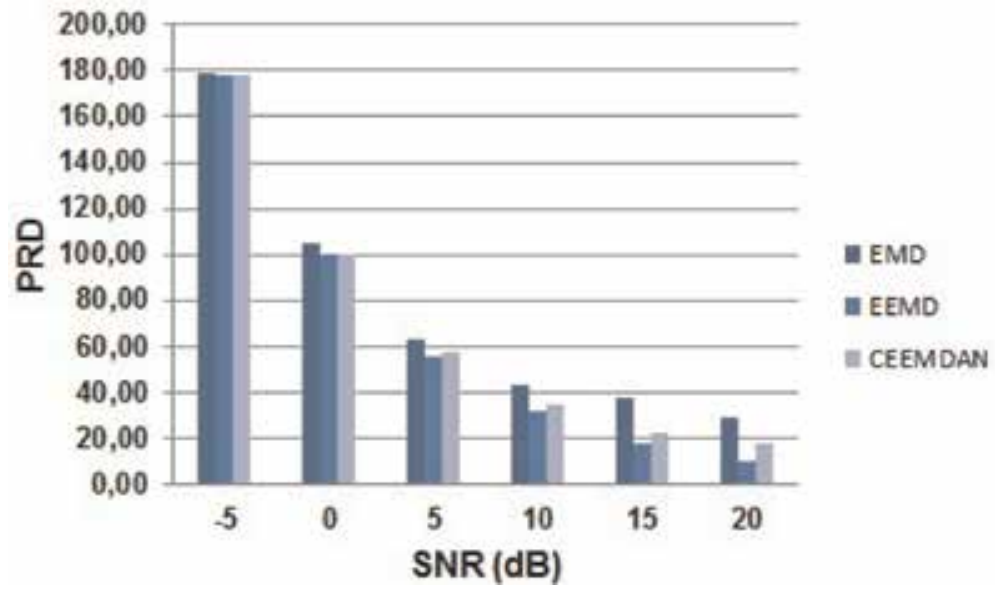

Figure 15.

PRD comparison of the different denoising methods at different SNR levels for the supraventricular arrhythmia ECG signal. 
Combination of the CEEM Decomposition with Adaptive Noise and Periodogram Technique...

DOI: http://dx.doi.org/10.5772/intechopen.86007

Section 2.3. Before applying the time-frequency techniques, these ECG signals were corrupted with different values of signal-to-noise ratio, varying from -5 to $20 \mathrm{~dB}$ with a $5 \mathrm{~dB}$ step.

Tables 6-10 report the obtained results of the mean square error (MSE), the root mean square error (RMSE), and the percent root mean square difference (PRD) after applying the three time-frequency methods (CW, PE, and SPWV) to the ECG signals, normal ECG, atrial fibrillation ECG, ventricular tachyarrhythmia ECG, malignant ventricular arrhythmia ECG, and supraventricular arrhythmia ECG, respectively.

\begin{tabular}{cccccccccc}
\hline \multirow{2}{*}{ SNR } & \multicolumn{3}{c}{ Choi-Williams } & \multicolumn{3}{c}{ Periodogram } & \multicolumn{3}{c}{ SPWV } \\
\cline { 2 - 9 } & MSE & RMSE & PRD & MSE & RMSE & PRD & MSE & RMSE & PRD \\
\hline-5 & 14.590 & 3.820 & 768.692 & 0.243 & 0.493 & 566.139 & 14.084 & 3.753 & 787.649 \\
\hline 0 & 2.247 & 1.499 & 338.207 & 0.029 & 0.171 & 254.753 & 1.910 & 1.382 & 332.290 \\
\hline 5 & 0.470 & 0.686 & 160.505 & 0.004 & 0.067 & 123.148 & 0.367 & 0.606 & 158.078 \\
\hline 10 & 0.124 & 0.352 & 80.891 & 0.001 & 0.030 & 62.893 & 0.092 & 0.303 & 79.802 \\
\hline 15 & 0.037 & 0.192 & 42.531 & 0.0002 & 0.015 & 33.357 & 0.027 & 0.163 & 42.003 \\
\hline 20 & 0.011 & 0.106 & 22.983 & 0.0001 & 0.008 & 18.122 & 0.008 & 0.090 & 22.712 \\
\hline
\end{tabular}

Table 6.

MSE, RMSE, and PRD of the normal ECG signal.

\begin{tabular}{cccccccccc}
\hline \multirow{2}{*}{ SNR } & \multicolumn{3}{c}{ Choi-Williams } & \multicolumn{3}{c}{ Periodogram } & \multicolumn{3}{c}{ SPWV } \\
\cline { 2 - 9 } & MSE & RMSE & PRD & MSE & RMSE & PRD & MSE & RMSE & PRD \\
\hline-5 & 13.303 & 3.647 & 271.222 & 0.252 & 0.502 & 151.833 & 13.340 & 3.652 & 270.031 \\
\hline 0 & 2.270 & 1.507 & 131.224 & 0.041 & 0.202 & 75.307 & 2.202 & 1.484 & 126.352 \\
\hline 5 & 0.478 & 0.691 & 67.090 & 0.008 & 0.090 & 39.165 & 0.458 & 0.676 & 64.774 \\
\hline 10 & 0.119 & 0.345 & 35.616 & 0.002 & 0.044 & 21.018 & 0.113 & 0.335 & 34.446 \\
\hline 15 & 0.033 & 0.181 & 19.362 & 0.001 & 0.023 & 11.501 & 0.031 & 0.176 & 18.746 \\
\hline 20 & 0.010 & 0.098 & 10.678 & 0.0001 & 0.012 & 6.367 & 0.009 & 0.095 & 10.345 \\
\hline
\end{tabular}

Table 7.

MSE, RMSE, and PRD of the atrial fibrillation ECG signal.

\begin{tabular}{cccccccccc}
\hline \multirow{2}{*}{ SNR } & \multicolumn{3}{c}{ Choi-Williams } & \multicolumn{3}{c}{ Periodogram } & \multicolumn{3}{c}{ SPWV } \\
\cline { 2 - 9 } & MSE & RMSE & PRD & MSE & RMSE & PRD & MSE & RMSE & PRD \\
\hline-5 & 65.469 & 8.091 & 423.064 & 1.295 & 1.138 & 220.730 & 65.501 & 8.093 & 418.365 \\
\hline 0 & 11.304 & 3.362 & 196.830 & 0.222 & 0.471 & 105.949 & 11.196 & 3.346 & 188.372 \\
\hline 5 & 2.351 & 1.533 & 97.777 & 0.046 & 0.213 & 53.838 & 2.327 & 1.526 & 93.922 \\
\hline 10 & 0.571 & 0.756 & 50.923 & 0.011 & 0.105 & 28.461 & 0.565 & 0.751 & 49.037 \\
\hline 15 & 0.154 & 0.393 & 27.357 & 0.003 & 0.054 & 15.431 & 0.153 & 0.391 & 26.384 \\
\hline 20 & 0.045 & 0.211 & 14.981 & 0.001 & 0.029 & 8.496 & 0.044 & 0.210 & 14.461 \\
\hline
\end{tabular}

Table 8.

MSE, RMSE, and PRD of the ventricular tachyarrhythmia ECG signal. 
Figures 16-20 illustrate the RMSE comparison results obtained by using the different time-frequency techniques (CW, PE, and SPWV), at a SNR interval varying from -5 to $20 \mathrm{~dB}$, to the following signals, respectively: normal ECG, atrial fibrillation ECG, ventricular tachyarrhythmia ECG, malignant ventricular arrhythmia ECG, and supraventricular arrhythmia ECG.

Figures 21-25 show the comparison PRD results of the three time-frequency techniques (CW, PE, and SPWV) to the five selected ECG signals.

\begin{tabular}{cccccccccc}
\hline \multirow{2}{*}{ SNR } & \multicolumn{3}{c}{ Choi-Williams } & \multicolumn{3}{c}{ Periodogram } & \multicolumn{3}{c}{ SPWV } \\
\cline { 2 - 9 } & MSE & RMSE & PRD & MSE & RMSE & PRD & MSE & RMSE & PRD \\
\hline-5 & 25.422 & 5.042 & 81.102 & 0.505 & 0.710 & 44.366 & 25.435 & 5.043 & 79.304 \\
\hline 0 & 4.654 & 2.157 & 42.667 & 0.092 & 0.303 & 23.665 & 4.621 & 2.150 & 40.959 \\
\hline 5 & 1.020 & 1.010 & 23.067 & 0.020 & 0.141 & 12.902 & 1.013 & 1.006 & 22.176 \\
\hline 10 & 0.257 & 0.507 & 12.679 & 0.005 & 0.071 & 7.127 & 0.255 & 0.505 & 12.200 \\
\hline 15 & 0.071 & 0.267 & 7.038 & 0.001 & 0.037 & 3.967 & 0.071 & 0.266 & 6.775 \\
\hline 20 & 0.021 & 0.145 & 3.929 & 0.0004 & 0.020 & 2.218 & 0.021 & 0.144 & 3.783 \\
\hline
\end{tabular}

Table 9.

MSE, RMSE, and PRD of the malignant ventricular arrhythmia ECG signal.

\begin{tabular}{cccccccccc}
\hline \multirow{2}{*}{ SNR } & \multicolumn{3}{c}{ Choi-Williams } & \multicolumn{3}{c}{ Periodogram } & \multicolumn{3}{c}{ SPWV } \\
\cline { 2 - 9 } & MSE & RMSE & PRD & MSE & RMSE & PRD & MSE & RMSE & PRD \\
\hline-5 & 0.410 & 0.640 & 1496.14 & 0.008 & 0.087 & 1300.33 & 0.407 & 0.638 & 1562.32 \\
\hline 0 & 0.071 & 0.267 & 618.458 & 0.001 & 0.035 & 541.681 & 0.068 & 0.262 & 618.646 \\
\hline 5 & 0.015 & 0.124 & 277.450 & 0.0002 & 0.016 & 244.693 & 0.015 & 0.121 & 277.768 \\
\hline 10 & 0.004 & 0.062 & 133.833 & 0.0001 & 0.008 & 118.663 & 0.004 & 0.060 & 134.056 \\
\hline 15 & 0.001 & 0.033 & 68.259 & $210^{-5}$ & 0.004 & 60.743 & 0.001 & 0.032 & 68.391 \\
\hline 20 & 0.0003 & 0.018 & 36.175 & $510^{-6}$ & 0.002 & 32.266 & 0.0003 & 0.017 & 36.250 \\
\hline
\end{tabular}

Table 10.

MSE, RMSE, and PRD of the supraventricular arrhythmia ECG signal.

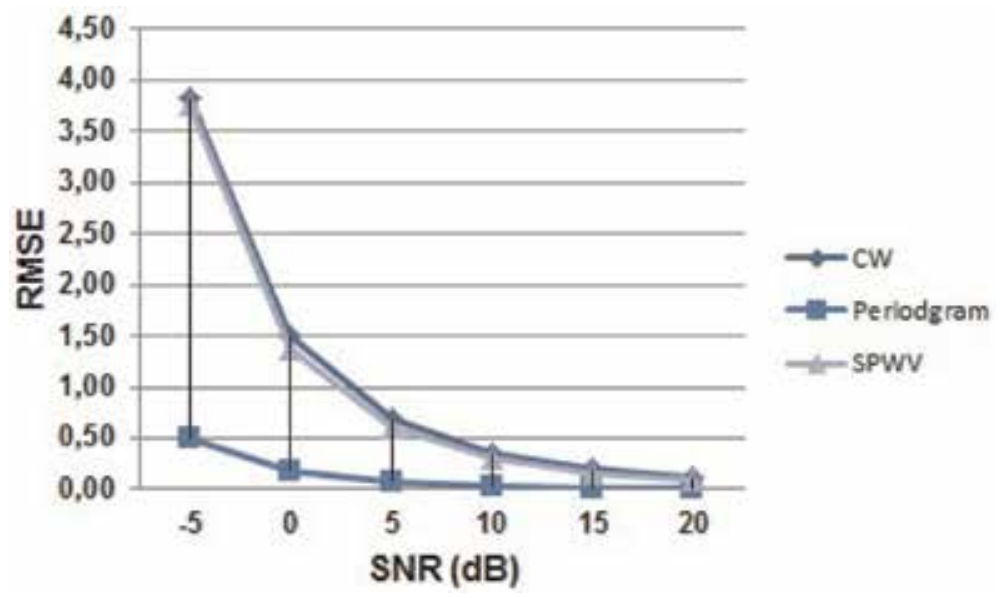

Figure 16.

RMSE comparison of the three time-frequency methods at different SNR levels for the normal ECG signal. 
Combination of the CEEM Decomposition with Adaptive Noise and Periodogram Technique... DOI: http://dx.doi.org/10.5772/intechopen.86007

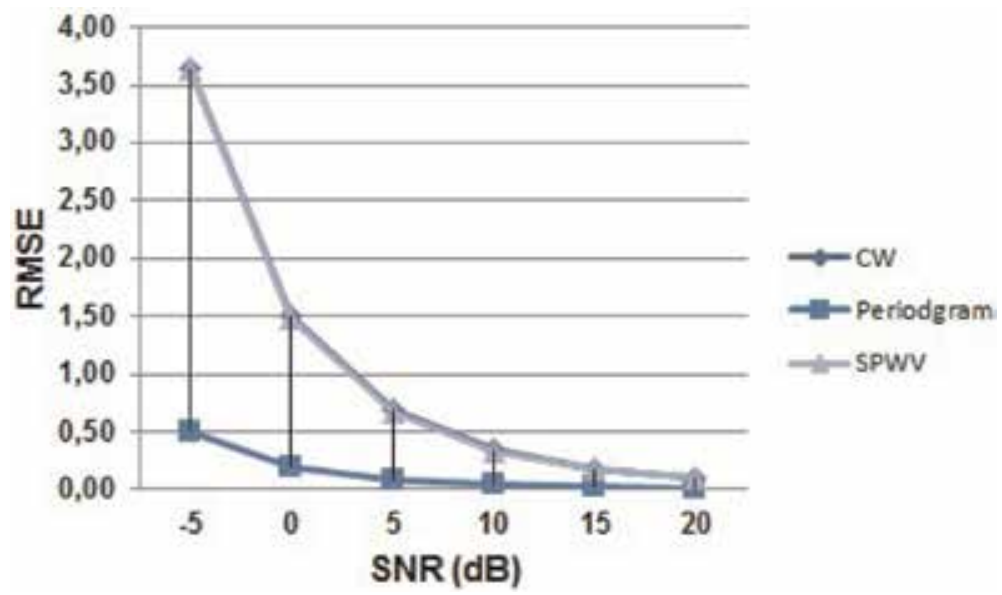

Figure 17.

RMSE comparison of the three time-frequency methods at different SNR levels for the atrial fibrillation ECG signal.

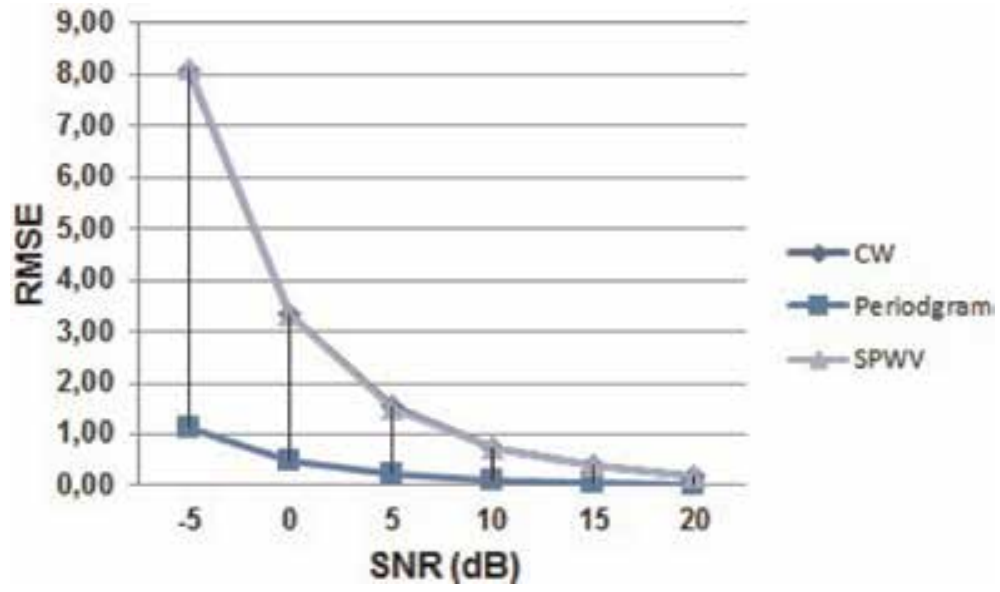

Figure 18.

RMSE comparison of the three time-frequency methods at different SNR levels for the ventricular tachyarrhythmia ECG signal.

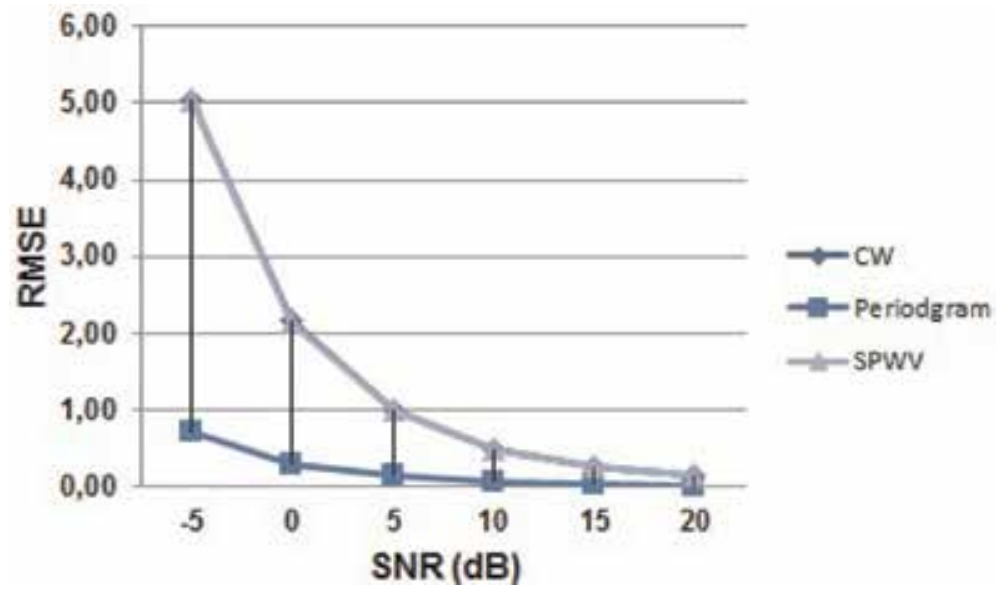

Figure 19.

RMSE comparison of the three time-frequency methods at different SNR levels for the malignant ventricular arrhythmia ECG signal. 


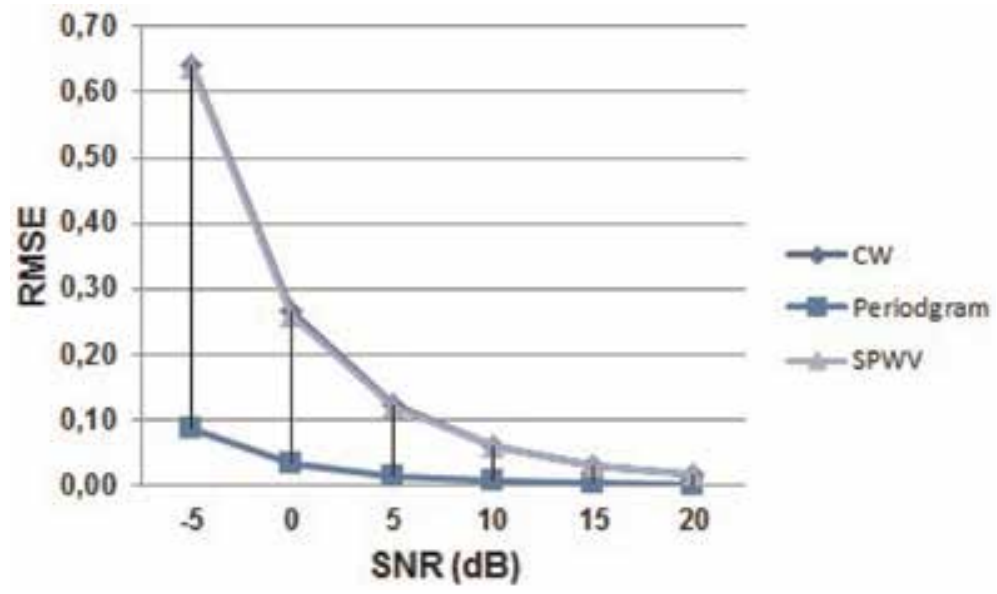

Figure 20.

RMSE comparison of the three time-frequency methods at different SNR levels for the supraventricular arrhythmia ECG signal.

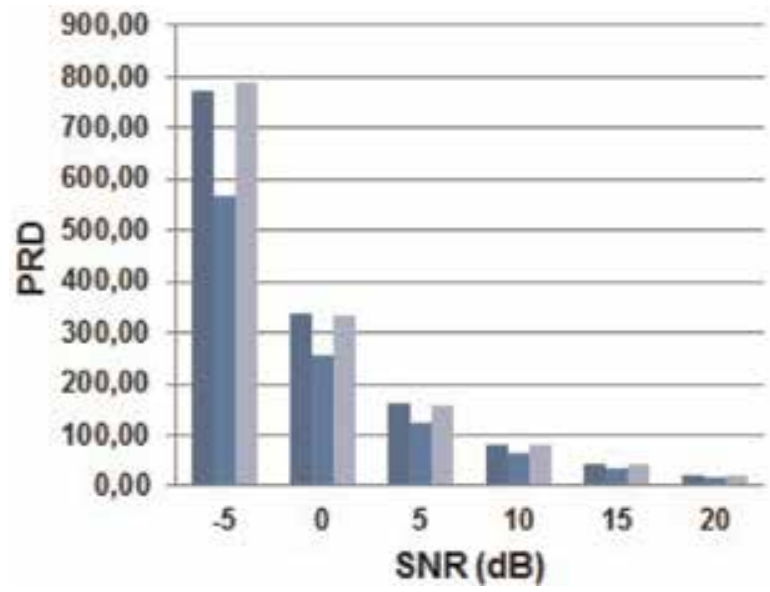

$\pm \mathrm{CW}$

=Periodgram

ISPW

Figure 21.

PRD comparison of the three time-frequency techniques (CW, PE, and SPWV) at different SNR levels for the normal ECG signal.

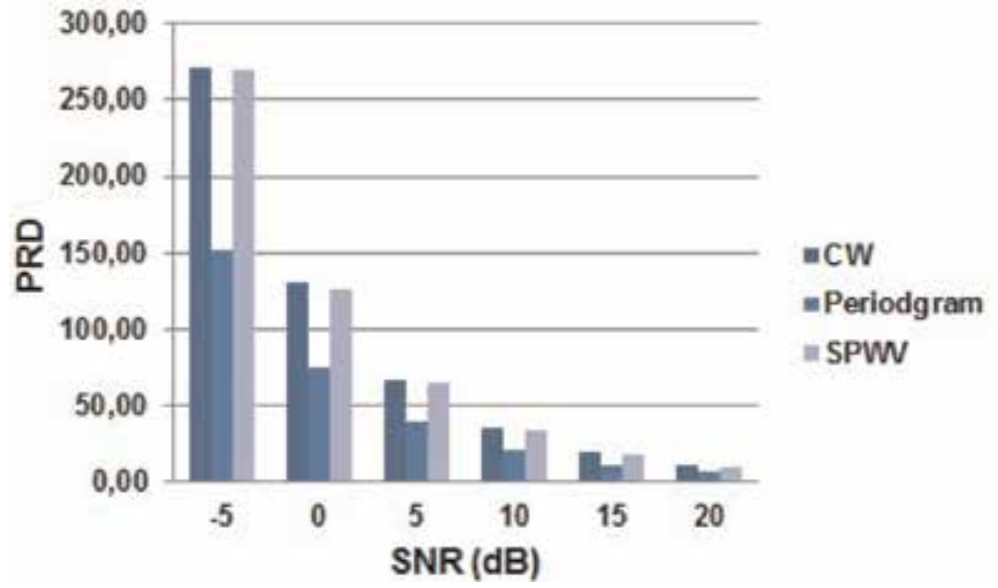

Figure 22.

PRD comparison of the three time-frequency techniques (CW, PE, and SPWV) at different SNR levels for the atrial fibrillation ECG signal. 
Combination of the CEEM Decomposition with Adaptive Noise and Periodogram Technique... DOI: http://dx.doi.org/10.5772/intechopen.86007

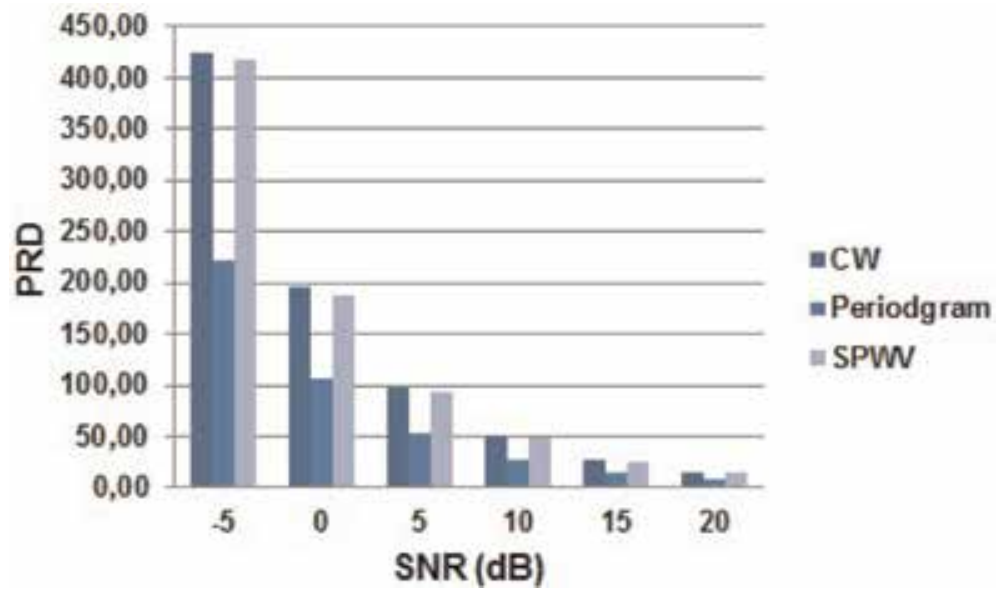

Figure 23.

$P R D$ comparison of the three time-frequency techniques (CW, PE, and SPWV) at different SNR levels for the ventricular tachyarrhythmia ECG signal.

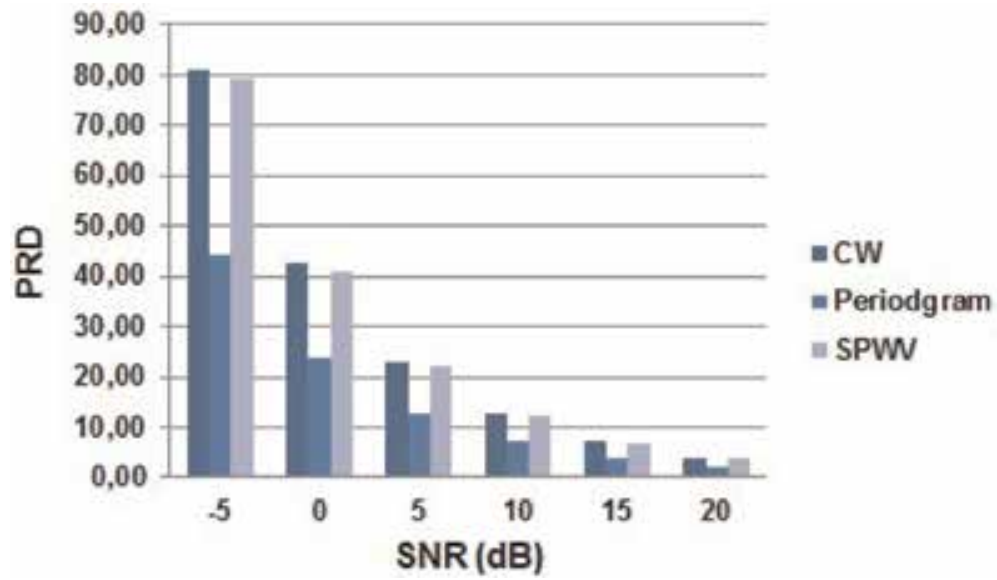

Figure 24.

PRD comparison of the three time-frequency techniques (CW, PE, and SPWV) at different SNR levels for the malignant ventricular arrhythmia ECG signal.

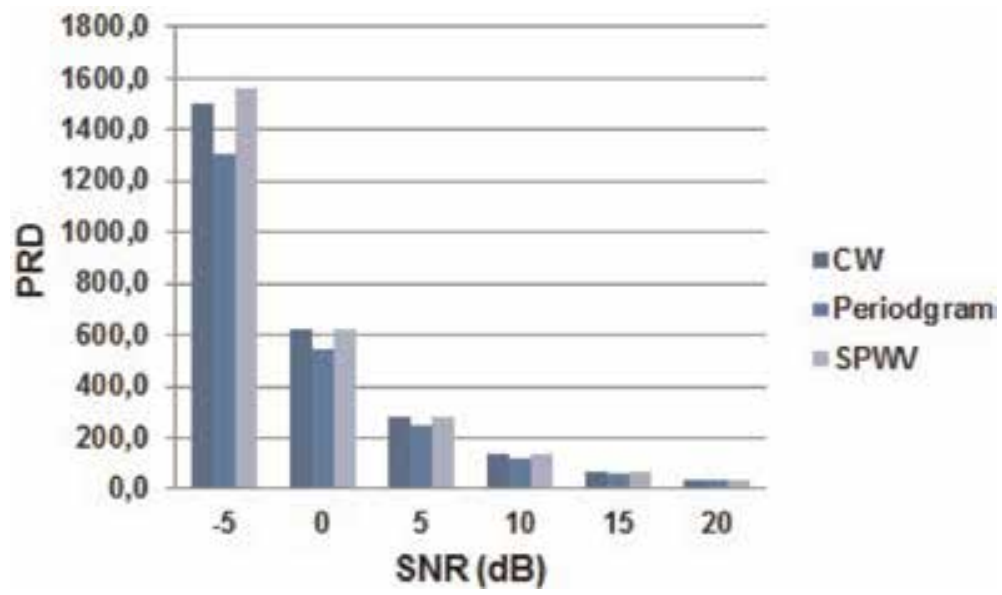

Figure 25.

PRD comparison of the three time-frequency techniques (CW, PE, and SPWV) at different SNR levels for the supraventricular arrhythmia ECG signal. 


\subsection{Discussion}

The study was divided to two separate steps. The first part involved a comparison between three denoising methods, empirical mode decomposition (EMD) and its two variants ensemble empirical mode decomposition (EEMD) and complete ensemble empirical mode decomposition with adaptive noise (CEEMDAN). We note from the obtained results (Tables 1-5 and Figures 6-15) that the EEMD and the CEEMDAN methods for the most selected ECG signals for the different signalto-noise ratio (SNR) values yield the smallest values of MSE, RMSE, and PRD compared to those obtained by the EMD method. Despite the EEMD method providing a slight advantage than CEEMDAN for a few degrees of SNR in some ECG signals, we concluded that the most optimal technique for denoising this type of biomedical signals is CEEMDAN, especially for the large number of iterations required in EEMD process.

The second part of study presents a comparison of the different results of the three time-frequency techniques Choi-Williams (CW), periodogram (PE), and smoothed pseudo Wigner-Ville (SPWV). These time-frequency techniques were applied to normal and abnormal ECG signals with different degrees of SNR varying from $-5 \mathrm{~dB}$ to $20 \mathrm{~dB}$. We note that the PE technique provides the best results; it furnishes the smallest values of MSE, RMSE, and PRD than those obtained by the two other techniques, CW and SPWV.

After these two steps, we concluded that a combination of the two techniques, CEEMDAN denoising method and PE time-frequency technique, would be ideal for the ECG signal analysis. The CEEMDAN method will be reserved for the pretreatment phase to filter the noise, and in the second phase, the PE technique will be applied to supply the evolution of the ECG signal fequential components over the time in order to provide a good diagnosis.

\section{Conclusion}

The work purpose was to conduct two comparative studies to determine the best techniques for ECG signal processing. The first one focused on the comparison between techniques aimed at preprocessing ECG signals, namely, denoising methods. The second one was to compare some time-frequency techniques that are intended to analyze these biomedical signals. The obtained results show that, in the first part, the CEEMDAN presents a high effectiveness in the noise elimination and, in the second one, the periodogram provides the best solution for analyzing ECG signals. We conclude that a combination of the CEEMDAN denoising method and the PE time-frequency technique can be a good issue in analyzing the ECG signals. 
Combination of the CEEM Decomposition with Adaptive Noise and Periodogram Technique... DOI: $h$ ttp://dx.doi.org/10.5772/intechopen.86007

\section{Author details}

Azzedine Dliou*, Samir Elouaham, Rachid Latif and Mostafa Laaboubi ESSI-LISTI Laboratory, National School of Applied Sciences, Ibn Zohr University, Agadir, Morocco

*Address all correspondence to: dliou.azzedine@yahoo.fr

\section{IntechOpen}

(C) 2019 The Author(s). Licensee IntechOpen. This chapter is distributed under the terms of the Creative Commons Attribution License (http://creativecommons.org/licenses/ by/3.0), which permits unrestricted use, distribution, and reproduction in any medium, provided the original work is properly cited. (c) BY 


\section{References}

[1] Jones SA. ECG Success Exercises in ECG Interpretation. Philadelphia: F. A. Davis Company; 2008

[2] Clifford GD, Azuaje F, McSharry PE. Advanced Methods and Tools for ECG Data Analysis. Norwood: Artech House, Inc; 2006

[3] Sende J. Guide Pratique ECG. ESTEM ed; 2003

[4] El B'charri O, Latif R, Elmansouri K, Abenaou A, Jenkal W. ECG signal performance de-noising assessment based on threshold tuning of dual-tree wavelet transform. Biomedical Engineering Online. 2017;16(1):26

[5] Huang NE, Shen Z, Long SR, Wu MC, Shih EH, Zheng Q, et al. The empirical mode decomposition method and the Hilbert spectrum for nonstationary time series analysis. Proceedings Royal Society of London. 1998;454A:903-995

[6] Liao A-H, Shen C-C, Li P-C. Contrast improvement by combining pulse inversion with EMD and EEMD. In: Proceedings of the IEEE International Ultrasonics Symposium (IUS'09); Rome, Italy. 2009. pp. 287-290

[7] Torres ME, Colominas MA, Schlotthauer G, Flandrin P. A complete ensemble empirical mode decomposition with adaptive noise. In: Proceedings of the 36th IEEE International Conference on Acoustics, Speech, and Signal Processing (ICASSP'11). Prague, Czech Republic: IEEE; 2011. pp. 4144-4147

[8] Bigan C, Woolfson MS. Timefrequency analysis of short segments of biomedical data. IEE Proceedings Science, Measurement \& Technology. 2000;147(6):368-373

[9] Clayton RH, Murray A. Estimation of the ECG signal spectrum during ventricular fibrillation using the fast Fourier transform and maximum entropy methods. In: Proceedings of the Computers in Cardiology. 1993.

pp. 867-870

[10] Cooley JW, Tukey JW. An algorithm for machine calculation of complex Fourier series. Mathematics of Computation. 1965;19:297-301

[11] Addison PS. Wavelet transforms and the ECG: A review. Physiological Measurement. 2005;26:R155-R199

[12] Mousa A, Saleem R. Using reduced interference distribution to analyze abnormal cardiac signal. Journal of Electrical Engineering. 2011;62(3): 168-172

[13] Dliou A, Latif R, Laaboubi M, Maoulainine FMR. Abnormal ECG signals analysis using non-parametric time-frequency techniques. February 2014;39(2):913-921

[14] Latif R, Laaboubi M, Aassif E, Maze G. Détermination de l'épaisseur d'un tube élastique à partir de l'analyse temps-fréquence de Wigner-Ville. Journal of Acta Acustica. 2009;95(5): 843-848

[15] Hussain ZM, Boashash B. IF estimation for multicomponent signals. In: Time-Frequency Signal Analysis and Processing: A Comprehensive Reference. Oxford: Elsevier; 2003. pp. 437-445

[16] Cohen L. Time-frequency distributions-A review. Proceedings of the IEEE. 1989;77(7):941-981

[17] Cohen L. Time-Frequency Analysis. Englewood Cliffs: Prentice Hall PTR; 1995

[18] Flandrin P. Time-Frequency/TimeScale Analysis. Boston: Academic Press; 1998 
[19] Physiobank, Physionet. Physiologic Signal Archives for Biomedical Research. 2005. Available from: http:// www.physionet.org/physiobank/ [Accessed: August, 2005]

[20] Elouaham S, Latif R, Nassiri B, Dliou A, Laaboubi M, Maoulainine F. Analysis electrocardiogram signal using ensemble empirical mode decomposition and time-frequency techniques. International Journal of Computer Engineering and Technology. 2013;4(2):275-289

[21] Flandrin P, Rilling G, Goncalves P. Empirical mode decomposition as a filter bank. IEEE Signal Processing Letters. 2004;11:112-114

[22] He X, Goubran RA, Liu XP.

Ensemble empirical mode decomposition and adaptive filtering for ECG signal enhancement. In:

Proceedings of 2012 IEEE International Symposium on Medical Measurements and Applications (MeMeA); 18-19 May 2012; Budapest, Hungary. 2012. pp. 1-5

[23] Yeh J-R, Shieh J-S, Huang NE. Complementary ensemble empirical mode decomposition: A novel noise enhanced data analysis method. Advances in Adaptive Data Analysis. 2010;2:135-156

[24] Colominas MA, Schlotthauer G, Torres ME. Improved complete ensemble EMD: A suitable tool for biomedical signal processing. Biomedical Signal Processing and Control. 2014;14:19-29

[25] Choi H, Williams W. Improved time-frequency representation of multicomponent signals using exponential kernels. IEEE Transactions on Signal Processing. 1989;37(6):

862-871

[26] Elouaham S, Latif R, Nassiri B, Dliou A, Laaboubi M, Maoulainine F. Analysis electroencephalogram signals using ANFIS and periodogram techniques. International Review on Computers and Software. 2013;8(12): 2959-2966 



\title{
Chapter 3
}

\section{Visualization of ECG Data on Variant Maps}

\author{
Zhihui Hou and Jeffrey Zheng
}

\begin{abstract}
This chapter presents variant maps for showing potential features in ECG data sets. The variant map is a visualization method different from a traditional ECG. In this chapter, the ECG data sets obtained by clinical ECG monitoring are used as the data source, and the corresponding variant maps are obtained by the variant statistics method. This chapter mainly introduces the variant statistics method about converting ECG data into variant maps. From sample results, various visual properties can be observed, and further explorations are required.
\end{abstract}

Keywords: variant maps, ECG data, visualization feature

\section{Introduction}

Today, people still are in a state of high cardiovascular disease incidence. The world is paying attention to cardiovascular diseases [1], mainly relying on the detection of ECG signals to promote the research of cardiovascular diseases. ECG signals are the product of a wide range of clinical ECG technologies. The electrocardiogram represents cardiac function and graphic signals [2], which are important means of diagnosing abnormal cardiac activity.

With the development of the information age, signal acquisition, data processing, and information analysis have become the main theme of scientific and technological development. In recent years, ECG signal research methods have made significant progress, such as the use of machine learning [3], clustering [4], partial fractal dimension [5], wavelet transform [6], and other methods for classification of arrhythmia detection [7]. Among the emerging ECG signal research methods, the most typical representative is the ECG scatter plot [8-10]. The ECG scatter plot observes the ECG signal in a new perspective, complementing traditional ECG detection.

The variant method is an emerging method for dealing with the phase change of the signal phase. Now the variant method has formed the theory of variant theory, variant logic function, and variant visualization method. In the 1990s, the application of variant method in the processing of binary image classification and conversion [11, 12]. In 2010, the variant method had been improved $[13,14]$. So far, the variant method has been continuously developed and applied to different data samples, quantum sequences [15, 16], random sequences [17], noncoding DNA [18-20], bat echo signals [21], ECG signals [22, 23], and variant construction [24].

The variant method can process massive random sequences and extract statistical measurement features from them. The ECG sequence is a natural random 
sequence. It is a good fit to apply the variant method to the statistical measurement characteristics of massive ECG sequences. It has research value. The main purpose of this chapter is to study the visual characteristics of ECG signals and to mine valuable information in ECG signals. This chapter introduces the overall architecture, module function, and core algorithm of the variant measurement system. The results of this study show that the variant maps provide a new observation angle for ECG signal feature detection, and it shows the resolution of ECG data in visual effect.

The experimental data samples and experimental results in this chapter will be introduced in the fourth part. The overall structure and workflow of the variant measurement system are introduced in the second part. The third part introduces core module function and algorithm and finally summarizes the research.

\section{Variant map for ECG}

\subsection{Overall structure}

The variant measurement system is divided into five modules as a whole, which are an input data source module, a variant processing module, a segmentation measurement module, a state statistics module, and an output variant map module. The structure of variant measurement is shown in Figure 1.

It can be seen in Figure 1 that each module has its specific function. The input data source module is mainly used to read the ECG sequence. The main function of the variant processing module is to discretize the continuous ECG sequence. Segmentation measurement module is to segment the sequence. The main function of the state statistics module is to count the state of the pseudogene sequence.

\subsection{Workflow chart}

The five modules in the variant measurement system are independent and connected. The workflow of the entire variant measurement system is shown in Figure 2.

As can be seen from Figure 2, the five modules of the variant measurement system are arranged in order. The output of the previous module is the input of the next module. The input and output of each module are as follows:

1. Input data source module: The input data set, the output is the length of N ECG sequence.

2. Variable value processing module: Input is ECG sequence of length $\mathrm{N}$, and the output is pseudogene sequence of length $\mathrm{N}$.

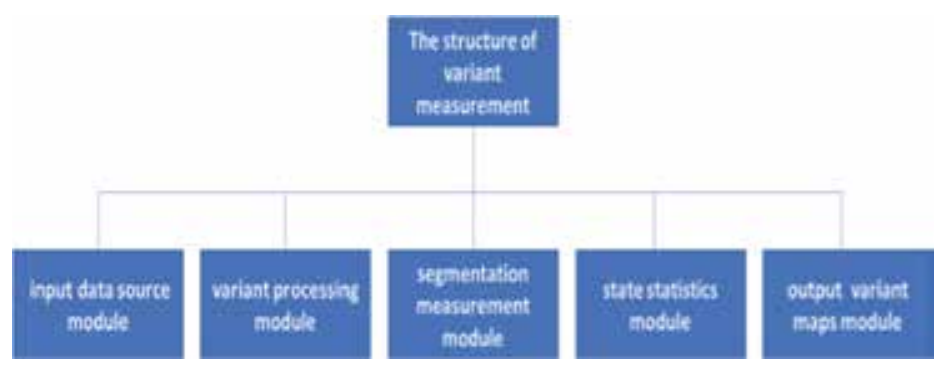

Figure 1.

The structure of variant measurement. 


\begin{tabular}{|c|c|c|c|c|}
\hline $\begin{array}{l}\text { input data } \\
\text { source } \\
\text { module }\end{array}$ & $\begin{array}{l}\text { Variant } \\
\text { processing } \\
\text { module }\end{array}$ & $\begin{array}{l}\text { segmentation } \\
\text { measuremen } \\
\text { tmodule }\end{array}$ & $\begin{array}{c}\text { state } \\
\text { Statistics } \\
\text { module }\end{array}$ & $\begin{array}{l}\text { output } \\
\text { variant maps } \\
\text { module }\end{array}$ \\
\hline
\end{tabular}

Figure 2.

The workflow of variant measurement.

3. Segmentation measurement module: Input segment length value "m," input pseudogene sequence of length $\mathrm{N}$, output is divided into $\mathrm{M}$ segment pseudogene sequence, where $\mathrm{N}=\mathrm{M}^{*} \mathrm{~m}$.

4. State statistics module: Input the pseudogene sequence with length $M$ $\left(\mathrm{N}=\mathrm{M}^{*} \mathrm{~m}\right)$, and output the corresponding variable measure value.

5. Variable value graph output module: Input variant measure, output variant ECG scatter plot.

\section{Core module}

\subsection{Variant processing module}

The core function of the module is to process successive ECG sequences into discrete 4-primary pseudogene sequences.

The variant processing module includes three submodules, a parameter setting submodule, a data discretization submodule, and a variant processing submodule. The three submodules are closely related, and the workflow of the module is shown in Figure 3.

As can be seen from the workflow chart of the variant processing module, the input and output relationship of the module is:

\section{Parameter setting:}

Sliding window value "W," $2 \leq W \leq N-1$; threshold "R," $R$ is a natural number greater than 0 .

Input:

The base sequence value of length $\mathrm{N}$ : Base_va $\}$ ue $=\left\{\mathrm{b}_{1}, \ldots, \mathrm{b}_{\mathrm{y}}\right\}$, where $\mathrm{N}$ is a positive integer.

Procedure:

A conversion sequence of length $\mathrm{N}$ : Trans_valur: $=\left\{\mathrm{t}_{0}, \ldots, \mathrm{r}_{\mathrm{M}-1}\right\}$, where $\mathrm{N}$ is a positive integer.

\section{Output:}

Pseudogene sequence of length $\mathrm{N}$ : Pseutogene_valu $=\left\{\mathrm{p}_{\|}, \ldots, \mathrm{P}_{\mathrm{N}}:\right\}, \mathrm{N}$ belongs to a positive integer, $\mathrm{P}_{i} \in$ Pseudogene_ralue and $\mathrm{P}_{\mathrm{i}}$ is an element in $\left[\mathrm{A}_{\mathrm{i}} \mathrm{G}, \mathrm{C}, \mathrm{T}\right]$.

The above is the overall workflow of the variant processing module. Since the variant processing module includes a parameter setting submodule, a data discretization submodule, and a variant conversion submodule, the functions of each submodule and its core algorithm will be specifically described below.

\subsubsection{Parameter setting submodule}

The parameter setting is to set the sliding window value "W" and the threshold " $R$ " two parameters. It should be noted that the parameters have dynamic adjustability. 


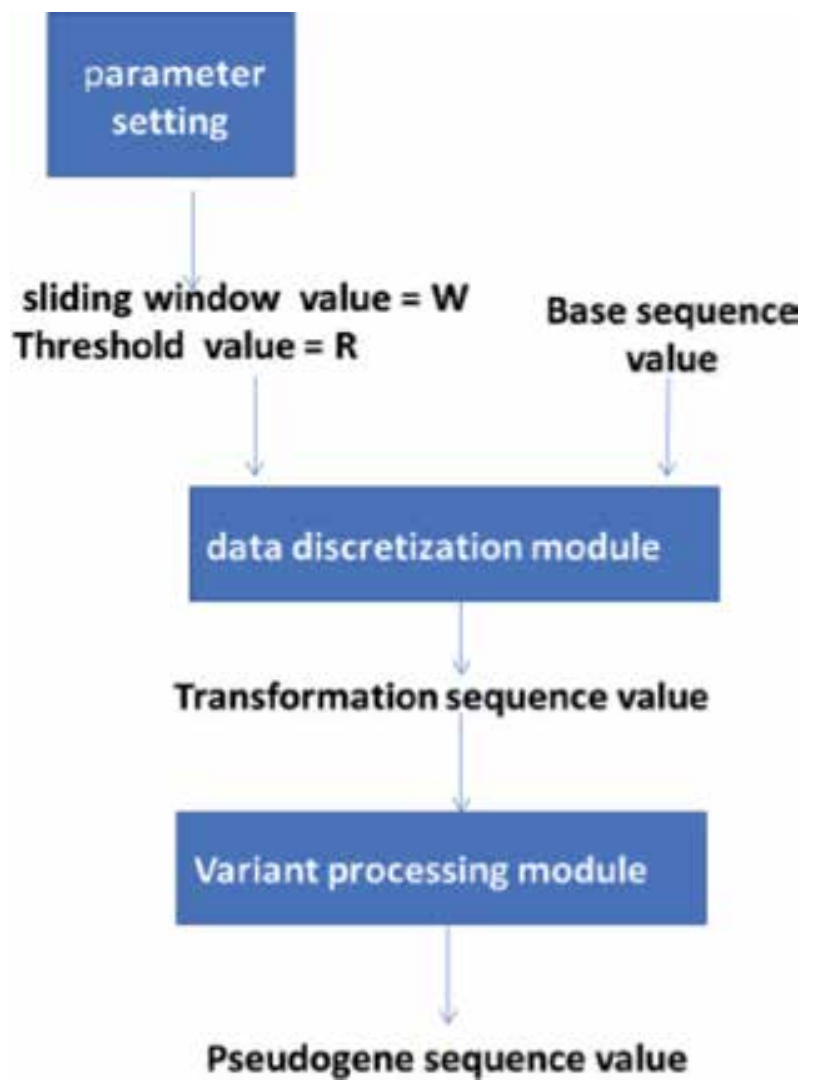

Figure 3.

The workflow of variant processing.

\subsubsection{Data discretization submodule}

The specific variable discretization algorithm is divided into three steps: the first step calculates the average sequence corresponding to the base sequence, the second step calculates the truncated average sequence corresponding to the base sequence, and the third step calculates the conversion sequence corresponding to the base sequence. The calculation of these three steps is as follows:

1. The first step is to calculate the average sequence corresponding to the base sequence. In the sliding window value, the sliding window is sequentially moved from the first position of the base sequence, one bit at a time, and the average value in the sliding window value obtained by each movement is calculated. The calculation process is:

\section{Input:}

The base sequence value of length $\mathrm{N}$ is Base_value $=\left\{\mathrm{b}_{1:}, \ldots, \mathrm{b}_{\mathrm{N}},\right\}, \mathrm{N}$ is a positive integer; the sliding window value is " $\mathrm{W}, " 2 \leq \forall \leq N-1$.

\section{Processing:}

Here is an example of the process of calculating a sliding window. Suppose the base sequence in the sliding window value " $W$ " is $b_{i}=\left\{b_{0}, \ldots, b_{w-1}\right\}$; then the average value of the sequence is $\overline{b_{1}}=\sum_{i=n}^{w-1} \overline{\mathrm{u}}$.

\section{Output:}

The average sequence of length $\mathrm{N}$ is $\mathrm{B}_{-}$value $\left.=\sqrt{\mathrm{b}_{0}}, \ldots, \overline{\mathrm{b}_{\mathrm{N}-1}}\right]$. 
2. The second step calculates the truncated average sequence corresponding to the base sequence. In the sliding window value, the sliding window is sequentially moved from the first position of the base sequence, one bit at a time, and the truncated average value in the sliding window value obtained by each movement is calculated. The calculation process is:

Input:

Base sequence value of length $\mathrm{N}$ : Base_value $=\left\{\mathrm{b}_{2}, \ldots, \mathrm{b}_{\mathrm{N}-1}\right\}, \mathrm{N}$ is a positive integer; threshold " $\mathrm{R}$," $\mathrm{R}$ is a natural number greater than 0 .

\section{Processing:}

Here is an example of the process of calculating a sliding window. Suppose the base sequence in the sliding window value " $W$ " is $b_{\mid}=\left\{b_{0}, \ldots, b_{n-1}\right\}$; then the maximum value of the elements in the sequence is $\mathrm{b}_{\text {nilix }}$, the minimum value is $\bar{b}_{1}^{\prime}=\left(b_{\max }-b_{\min }\right) \times \frac{\left(\mathrm{B}_{2}\right.}{2}$, then the truncated mean of the sequence is $B_{-}$value ${ }^{\prime}=\left\{\bar{b}_{0}, \ldots, \overline{b_{N-1}}\right\}$.

\section{Output:}

Truncated average sequence of length $\mathrm{N}$ : Base_value $=\left\{\mathrm{b}_{9}, \ldots, \mathrm{b}_{\mathrm{N}-1}\right\}$.

3. The third step calculates the conversion sequence corresponding to the base sequence:

\section{Input:}

Threshold " $\mathrm{R}$," $\mathrm{R}$ is a natural number greater than 0 ;

the base sequence of length $\mathrm{N}$ is Base_value $=\left\{\mathrm{b}_{0}, \ldots, \mathrm{b}_{\mathrm{N}-1}\right\}$;

the average sequence of length $\mathrm{N}$ is $\mathrm{B}_{-}$value $=\left\{\overline{\mathrm{b}_{0}}, \ldots, \overline{\mathrm{b}_{\mathrm{N}-1}} ;\right.$; and

the truncated average sequence of length $\mathrm{N}$ is Base_value $=\left\{\mathrm{b}_{\varphi}, \ldots, \mathrm{b}_{\mathrm{N}-1}\right\}$.

\section{Processing:}

For example, calculation of the $i$-th element $b_{i}$ in the base sequence to the $i$-th element $t_{j}$ in the conversion sequence: $t_{i}=\left(b_{1}-\bar{b}_{1}\right) /\left(\overline{b_{1}}{ }^{\prime} \times R\right)$.

\section{Output:}

Conversion sequence of length $\mathrm{N}$ is Trans_value $=\left\{\mathrm{t}_{0}, \ldots, \mathrm{t}_{\mathrm{N}-1}\right\}$, where $\mathrm{N}$ is a positive integer.

\subsubsection{Variant processing submodule}

The variant processing submodule is for processing the conversion sequence into a corresponding pseudogene sequence. The conversion rule is based on the threshold value, dividing the number axis into four intervals, and the four intervals correspond to the four primitives of the gene sequence: $A, G, C$, and $T$. When the conversion value is greater than or equal to the threshold, the conversion value is defined as $\mathrm{A}$. When the conversion value is less than or equal to the negative threshold, the conversion value is defined as T. When the conversion value is greater than 0 and less than the threshold, the conversion value is defined as $\mathrm{G}$, and when the conversion value is less than 0 . When the value is greater than the negative threshold, the conversion value is defined as $\mathrm{C}$; the conversion rules are as follows:

\section{Input:}

A sequence of converted values of length $\mathrm{N}$ : Trans_value $=\left\{\mathrm{t}_{0}, \ldots, \mathrm{t}_{\mathrm{N}-1}\right\} ; \mathrm{N}$ is a positive integer; threshold " $R$," $R$ is a natural number greater than 0 .

\section{Processing:}

For example, conversion rule between the $\mathrm{i}$-th element $t_{i}$ and the threshold in the conversion sequence:

$$
\begin{array}{ll}
\text { if } t_{1} \geq R \geq 0: a \rightarrow A & \text { if } R>t_{i}>0: a \rightarrow G \\
\text { if }-R \leq t_{i}<0: t_{i} \rightarrow C & \text { if } t_{j} \leq-R<0: t \rightarrow T
\end{array}
$$




\section{Output:}

A pseudogene sequence of length $\mathrm{N}$ Psendagene_value $=\left\{p_{q}, \ldots . p_{N_{1}}\right\}$, where $\mathrm{N}$ is a positive integer.

\subsection{Segmented measurement module}

The segmentation measurement module is to segment the pseudogene sequence. The function of this step is simple, but it is essential to prepare for the state statistics module. It should be noted that the segmentation measurement method here is different from the sliding window value in the variable value processing module. The principle of sliding window operation in the variable value processing module is to perform correlation measurement in order of 1 interval and sliding window value as unit length. The segmentation measurement is based on the segment length and sequentially segments the data sequence. For example, when the segment length is $\mathrm{m}$, the pseudogene sequence of length $\mathrm{N}$ can be divided into $\mathrm{M}$ segments, $\mathrm{N}=\mathrm{M}^{*} \mathrm{~m}$. The workflow of segmented measurement is in Figure 4.

The input and output relationship of this module is:

\section{Parameter setting:}

The segment length value is recorded as “m," $4 \leq \mathrm{m}$.

Input:

Segmentation length value " $m$ "; pseudogene sequence of length $\mathrm{N}$ : Psendogene $=\left\{p_{n}, \ldots, p_{1}\right\}$.

\section{Processing:}

Segmenting the pseudogene sequence of length $\mathrm{N}$ in turn at intervals of segment length $\mathrm{m}$.

\section{Output:}

The segmentation length of $\mathrm{m}$ is divided into $\mathrm{M}$ groups of pseudogene sequences

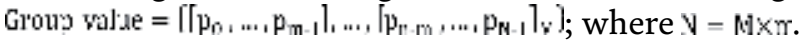

\subsection{Variant state statistics module}

This module statistically analyzes the sequence mathematically, revealing the patterns in the data and the relationship between the data. The module uses

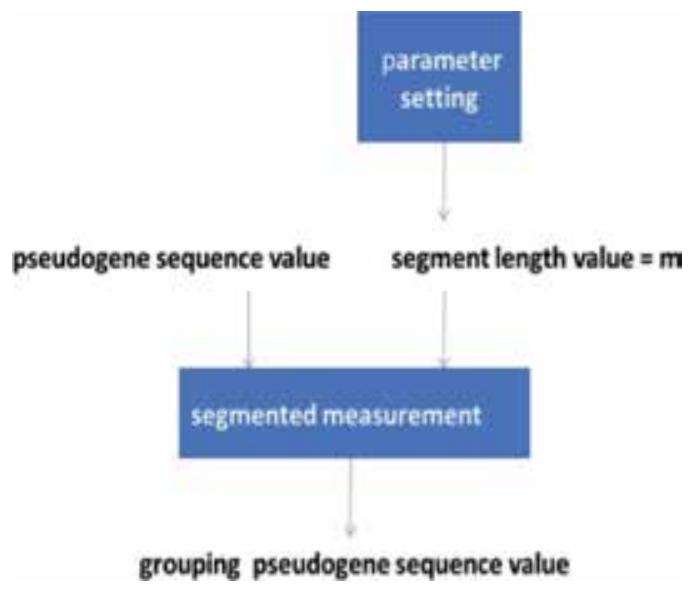

Figure 4 .

The workflow of segmented measurement. 


\section{grouping pseudogene sequence value}

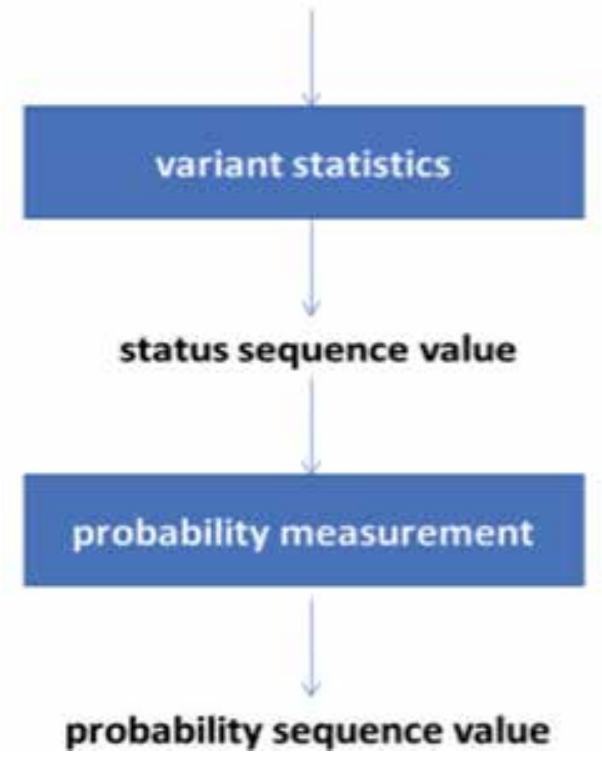

Figure 5

The workflow of variable state statistics.

statistical ideas to calculate the measure values of the various primitives of $A, G$, $\mathrm{C}$, and $\mathrm{T}$ in the pseudogene sequence. The measurement method is to count the number of each primitive in each group in the grouping sequence of the pseudogene sequence and mark the obtained value as a state statistical sequence. The workflow chart is shown in Figure 5.

As can be seen from Figure 5, the input and output relationship of the module is:

Input:

Segment length with $\mathrm{m}$; pseudogene sequence

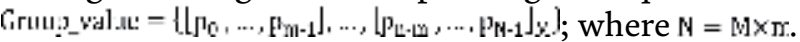

Processing:

Processing includes variant conversion statistics and variant probability measurement.

Output: Probability measure sequence.

The rules for variant conversion statistics and variant probability measurement are defined as follows.

\subsubsection{Variant conversion statistics}

The process of variant conversion statistics is illustrated by taking the $\mathrm{i}$-th group in the grouped pseudogene sequence as an example:

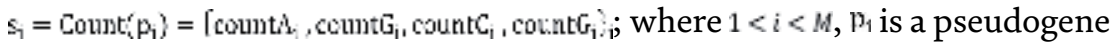
sequence consisting of all pseudogenes in the $i$-th group.

count $A_{i}$ represents the value of the number of A primitives in the $i$-th group;

count $\int_{r_{\mathrm{i}}}$ represents the value of the number of $\mathrm{G}$ primitives in the $\mathrm{i}$-th group;

count $C_{i}$ represents the value of the number of $C$ primitives in the $i$-th group; and

count $\mathrm{T}_{\mathrm{i}}$ represents the value of the number of $\mathrm{T}$ primitives in the $\mathrm{i}$-th group.

Taking the $i$-th group as an example, the state measurement sequence S_valuc $=\left\{s_{1}, \ldots, s_{M}\right\}$ can be obtained by analogy. 


\subsubsection{Variant probability measurement}

The following describes the process of probability measurement by taking the i-th group in the pseudogene sequence as an example:

$$
\begin{aligned}
& \mathrm{S}_{\mathrm{i}}=\operatorname{Count}\left(\mathrm{p}_{\mathrm{i}}\right) \\
& =\left\{\operatorname{count}_{\mathrm{i}}, \operatorname{count} \mathrm{G}_{\mathrm{i}}, \operatorname{count} \mathrm{C}_{\mathrm{i}}, \operatorname{count} \mathrm{T}_{\mathrm{i}}\right)_{\mathrm{i}} \\
& \operatorname{count}_{\mathrm{i}}=\operatorname{count} \mathrm{A}_{\mathrm{i}} /\left(\operatorname{count} \mathrm{A}_{\mathrm{i}}+\operatorname{count} \mathrm{C}_{\mathrm{i}}\right) \\
& \operatorname{count} \mathrm{G}_{\mathrm{i}}=\operatorname{count} \mathrm{G}_{\mathrm{i}} /\left(\operatorname{count} \mathrm{T}_{\mathrm{i}}+\operatorname{count} \mathrm{G}_{\mathrm{i}}\right) \\
& \operatorname{count} \mathrm{C}_{\mathrm{i}}=\operatorname{count} \mathrm{C}_{\mathrm{i}} /\left(\operatorname{count} \mathrm{A}_{\mathrm{i}}+\operatorname{count} \mathrm{C}_{\mathrm{i}}\right) \\
& \operatorname{count}_{\mathrm{i}}=\operatorname{count} \mathrm{T}_{\mathrm{i}} /\left(\operatorname{count} \mathrm{T}_{\mathrm{i}}+\operatorname{count} \mathrm{G}_{\mathrm{i}}\right)
\end{aligned}
$$

Taking the i-th group as an example, the probability measurement sequence $s=\left\{s_{1}, \ldots, s_{\mathrm{M}}\right]$ can be obtained by analogy.

\section{Sample results and brief analysis}

\subsection{Data source description}

ECG data samples from the First People's Hospital of Yunnan Province. This batch of data sets was initially analyzed by hospital experts. In order to facilitate the experimental research, an ECG database was established to classify ECG data. Among them, the normal ECG data is about $138 \mathrm{MB}$, and the abnormal ECG data is about $362 \mathrm{MB}$. The data samples obtained by collation are shown in Figure 6.

As can be seen in Figure 6, ECG data belongs to multivalued data and has a plurality of different attribute values, including pr interval, qt interval, $p$ wave, qrs wave, and the like. In the medical field, the diagnosis of P-wave signals is a key point and difficulty in research. The $\mathrm{P}$ wave is the key to the diagnosis of arrhyth-

\begin{tabular}{|c|c|c|c|c|c|c|c|c|c|c|c|c|c|c|c|}
\hline d & study jretance jo & hest joste & $x$ & gt & D & pats & os yidth & P.yidh & tyidth & tyidth & gts & Ni & ns & sol & 55 \\
\hline$t$ & Ecc5201404:500013 & 190 & $1 \%$ & 366 & 62 & 83 & $\%$ & 62 & 0 & 423 & 124 & 0.12 & 1.15 & 199 & 1.35 \\
\hline 2 & ECC552014050700003 & 117 & 124 & 294 & 48 & 34 & 30 & 40 & 0 & 363 & 122 & 0.22 & 0.25 & 13 & 0.66 \\
\hline 3 & NEDEPS2013092100066 & 81 & 156 & 380 & 16 & 85 & 50 & 16 & 0 & 417 & 94 & 0.02 & 0.41 & 1 & 0.37 \\
\hline 4 & XTS\$2013:28000004 & 61 & 144 & 352 & 110 & 94 & 56 & 110 & 0 & 354 & 96 & 0.1 & 0.93 & 153 & 0.84 \\
\hline 5 & Xot329:4122850009 & 124 & 194 & 32 & 47 & 90 & 39 & 4 & 0 & 375 & $1 \%$ & 0.27 & 0.59 & 1.23 & 0.6 \\
\hline 6 & EC65201*072500005 & 123 & 128 & 252 & 146 & 68 & 30 & 146 & 0 & 325 & 36 & 0.08 & 0.59 & 0.52 & 0.26 \\
\hline 7 & Ex 202015031100001 & 64 & 90 & 422 & 100 & 62 & 93 & 100 & 0 & 411 & 52 & 0.18 & 0.08 & $a .38$ & 0.06 \\
\hline 8 & EC2520150412000002 & 100 & 92 & 298 & 127 & 62 & 51 & 127 & 0 & 345 & 56 & 0.37 & 0 & 0 & 0 \\
\hline 9 & Der:S201421200002 & 85 & 156 & 280 & -27 & 100 & 12 & -27 & 0 & 322 & 90 & 0.93 & 0.29 & 0.52 & 0 \\
\hline 10 & Ex:s2015031700002 & 136 & 118 & 29 & 154 & B & 31 & 154 & 0 & 310 & $\%$ & 0.14 & 0 & 0 & 0 \\
\hline 11 & NeDePS2014031700204 & 110 & 96 & 293 & 76 & 76 & 90 & 76 & 0 & 333 & 78 & 0.79 & 0 & 0.72 & 3.71 \\
\hline 12 & XTs52013122100006 & 92 & 128 & 506 & $n 2$ & 122 & 58 & $n$ & 0 & 555 & 112 & 0.51 & 0.13 & 128 & 2.5 \\
\hline 13 & NEDEPS201409240176 & 83 & $1 \%$ & 310 & 152 & $x$ & 90 & 162 & 0 & $3 *$ & 128 & 0.54 & 0.26 & 0 & 2.18 \\
\hline 14 & GaKs2013072400002 & $6 !$ & 132 & 412 & S1. & 78 & 25 & 51 & 0 & 414 & 86 & 0.42 & 0.14 & 2.25 & 0.87 \\
\hline 15 & NevePS201501260007n & 46 & 150 & 308 & 90 & Bs & 53. & 90 & 0 & 401 & 34 & 0.18 & 0.27 & 117 & 0.57 \\
\hline s. & 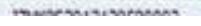 & me & mane & wh & 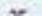 & $\infty$ & and & $\sim$ & a & m & $\operatorname{man}$ & $n=$ & and & $n \in$ & n \\
\hline
\end{tabular}
mia; as shown in Figure 7, it is the normal ECG signal that marks P.

Based on the above background, this chapter selects the P-wave data in the ECG data provided by the First People's Hospital of Yunnan Province to perform variable value visualization analysis. In order to ensure the rigor of the experiment, the normal $\mathrm{P}$ wave and the abnormal $\mathrm{P}$ wave of the same data amount were selected

Figure 6.

Sample of ECG data. 


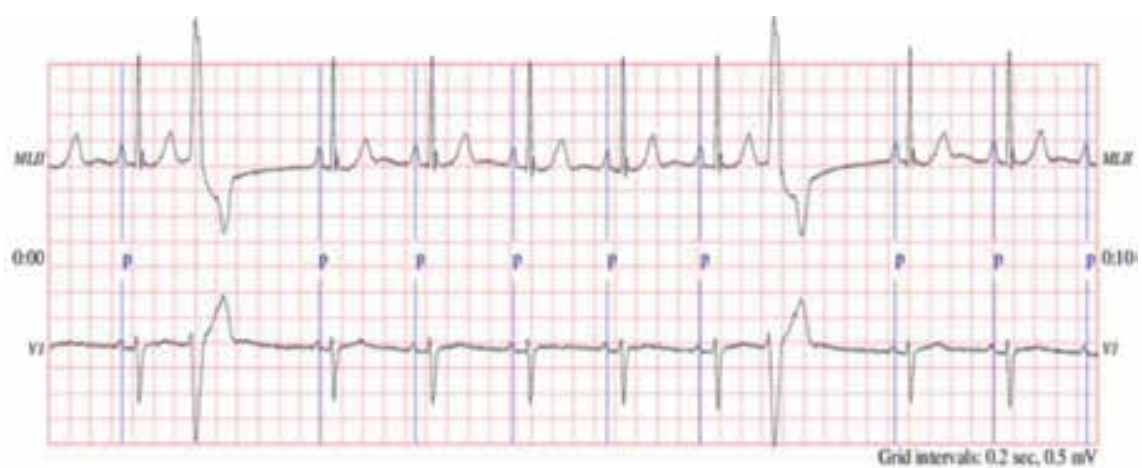

Figure 7.

The normal ECG signal that marks $P$.

for research. By comparing the variant maps between the normal $\mathrm{P}$ wave and the abnormal $\mathrm{P}$ wave, the useful information in the ECG data is mined.

\subsection{Meaning of selected variable value map}

\section{Input:}

data source and parameter value; the data source is a normal P-wave ECG sequence of length 10,254, an abnormal P-wave ECG sequence of length 10,254; the parameter is the sliding window value "W," and the threshold " $R$," a segment length value " $m$ ";

Processing:

The process is completed by the variant measurement system.

Output:

Variant maps: the $\mathrm{X}$-axis represents the probability measure of $\mathrm{G}$ in the four primitives $\mathrm{A}, \mathrm{G}, \mathrm{C}$, and $\mathrm{T}$, and the $\mathrm{Y}$-axis represents the probability measure of $\mathrm{C}$ in the four primitives $A, G, C$, and $T$. Marked on the variable map as $X=S t(G), Y=S t(C)$.

\subsection{Visualization features}

Figure 8 shows an example of normal $\mathrm{P}$-wave and anomalous $\mathrm{P}$-wave variation map. This example is a variable value map obtained under the condition that the

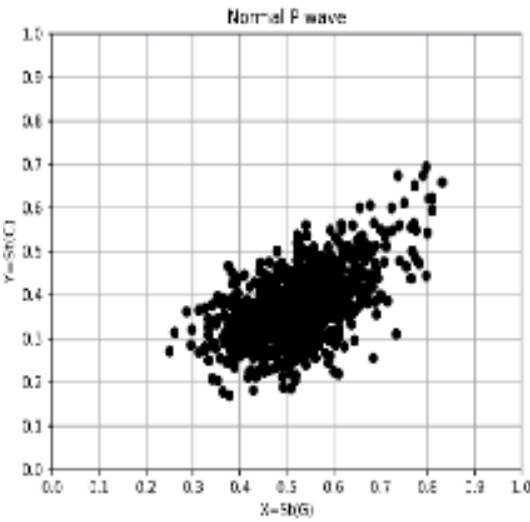

(n)

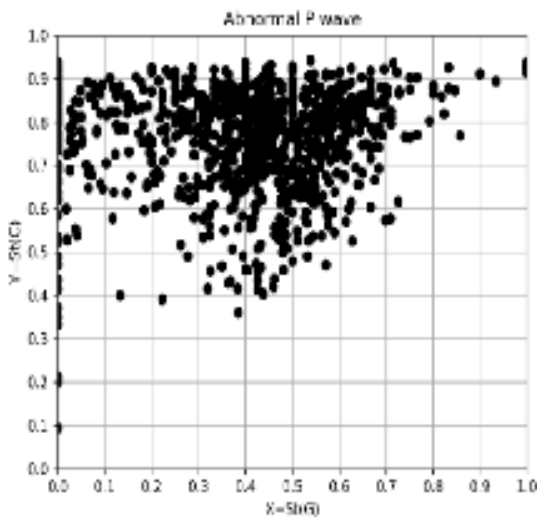

(b)

Figure 8.

Normal P-wave and abnormal P-wave characteristics. (a) Normal P-wave variant map. (b) Abnormal $P$-wave variant map. 
parameter sliding window value $\mathrm{W}=24$, the threshold $\mathrm{R}=0.85$, and the segment length value $\mathrm{m}=100$ are selected. It can be seen that there are obvious differences in the shape characteristics of the normal P-wave and the abnormal P-wave scatter cluster, and the distribution characteristics of the scatter cluster between the two are also different. The normal $\mathrm{P}$-wave characteristics are mainly concentrated in the interior of the quadrilateral formed by “ $(0.3,0.4),(0.4,0.1),(0.8,0.4),(0.8,0.7)$." The abnormal P-wave characteristics are mainly concentrated inside the triangle formed by “ $(0,1),(0.4,0.4),(1,1)$."

In order to better display the variant features, the following will be shown as an example of the visualization results under different "m" values (Figure 9).

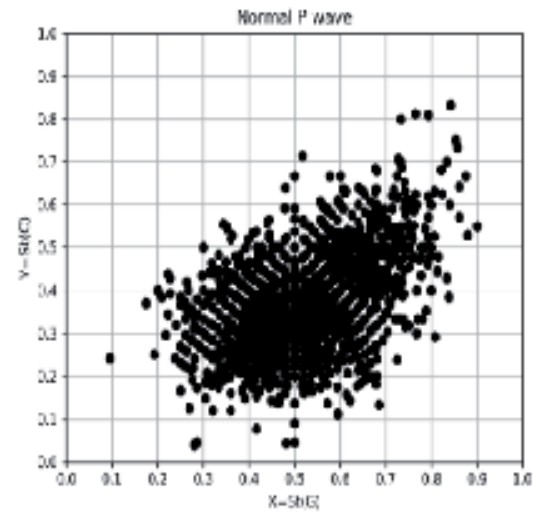

$\left(\mathrm{a}_{1}\right)$

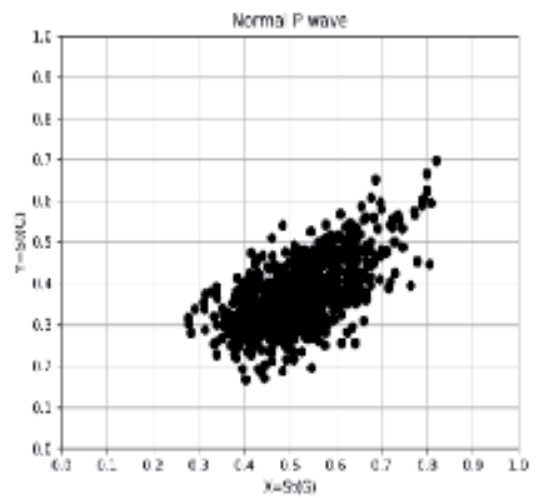

(as)

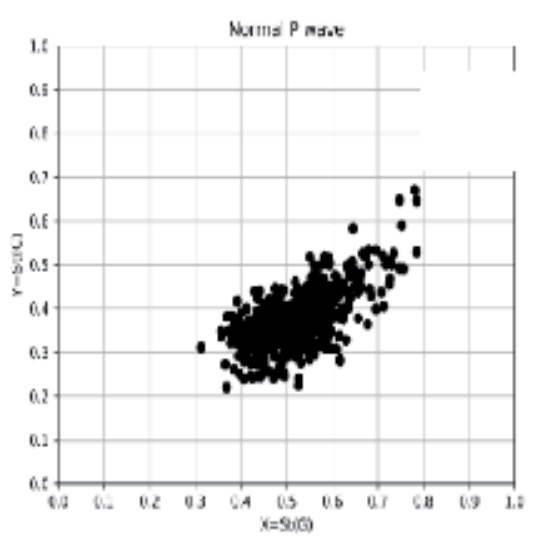

(ns

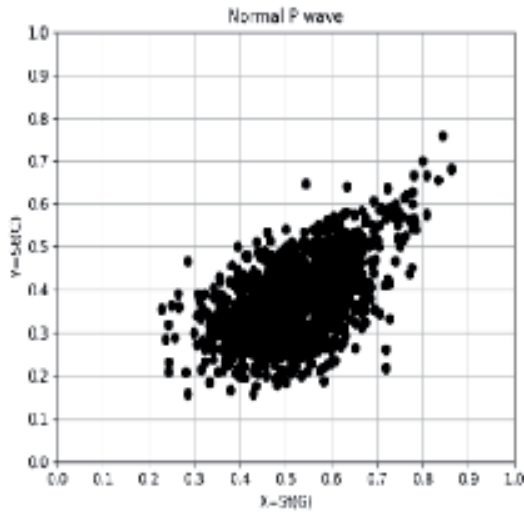

(az)

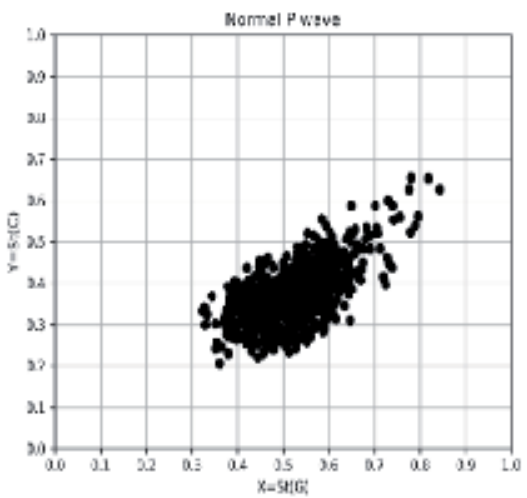

(a)

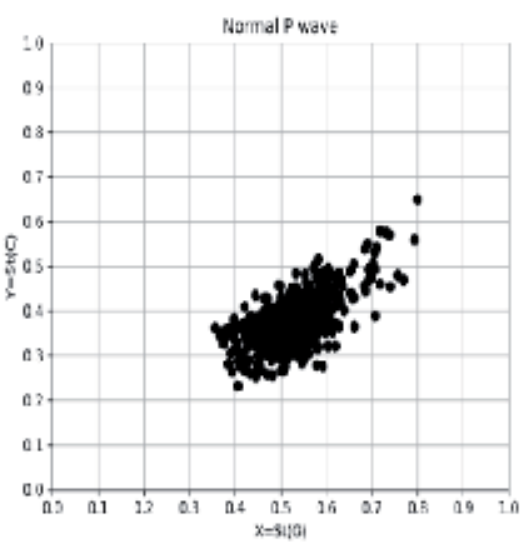

(ม6) 


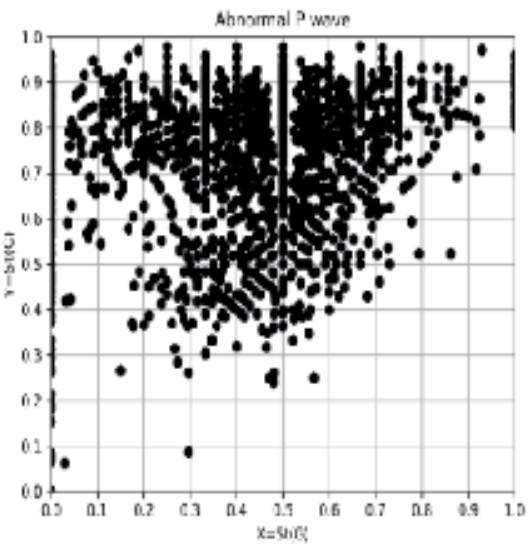

(b)

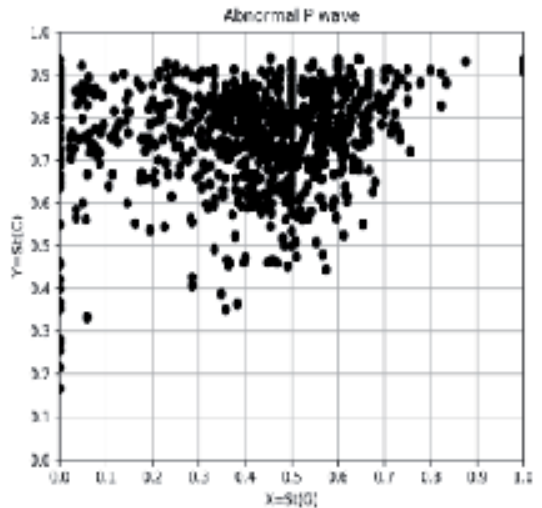

(b.)

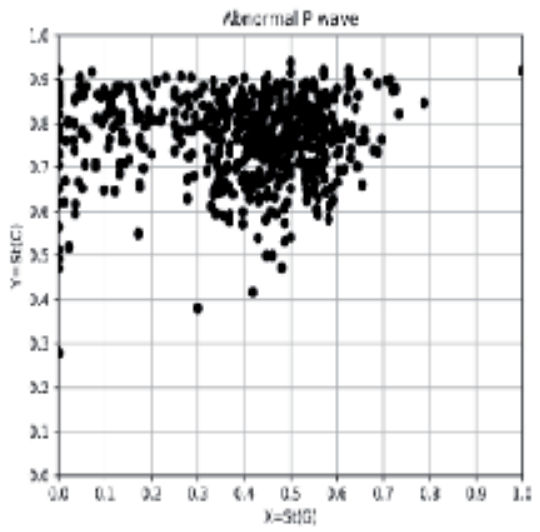

(bs)

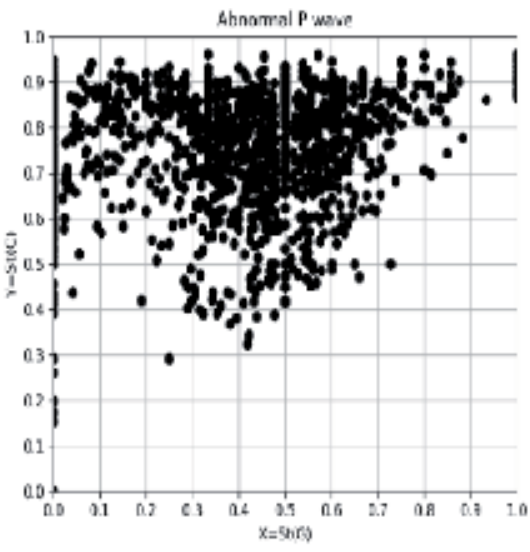

(b)

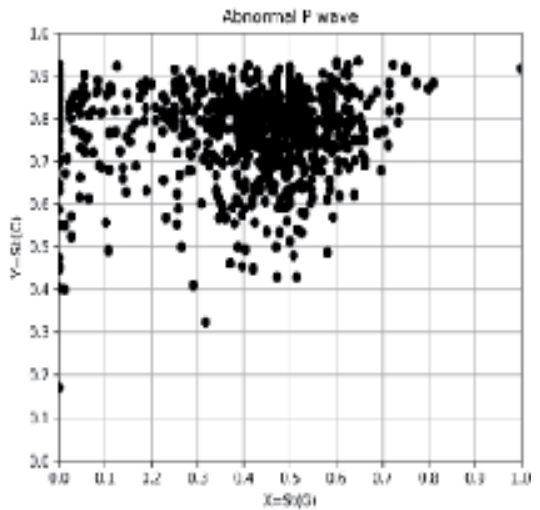

(bu)

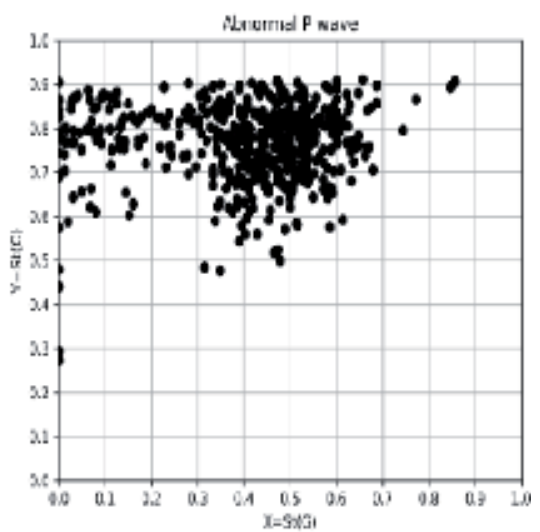

(bu)

Figure 9.

$A$ list of variant maps on parameters $M=\{50,80,110,140,170,200\} ;\left(a_{1}\right)-\left(a_{6}\right)$ is a list of normal $P$ wave; and $\left(b_{1}\right)-\left(b_{6}\right)$ is a list of abnormal $P$ wave. $\left(a_{1}\right) m=50,\left(a_{2}\right) m=80,\left(a_{3}\right) m=110,\left(a_{4}\right) m=140,\left(a_{5}\right) m=170$, and $\left(a_{6}\right) m=200$; and $\left(b_{1}\right) m=50,\left(b_{2}\right) m=80,\left(b_{3}\right) m=110,\left(b_{4}\right) m=140,\left(b_{5}\right) m=170$, and $\left(b_{6}\right) m=200$.

\section{Summary}

This chapter is closely related to the measurement model, processing method, and variant maps to study ECG signals. To some extent, the variant maps and the traditional clinical ECG can be compared: 
1. The electrocardiogram is a characteristic map obtained by processing the individual ECG signal. The variant maps, which mainly target massive ECG signals, can process individual ECG signals and can also process cluster ECG signals to provide visual analysis of points and surfaces.

2. The waveform features on the electrocardiogram have strong professionalism and complexity, while variant maps show waveform features from another perspective in the form of scatter clusters; variant maps visualization features are simple and clear. Nonprofessional ECG experts can also see the difference between normal and abnormal ECG characteristics.

The experimental results in this chapter demonstrate the visual characteristics of the differences in ECG data, giving a simple and clear visual experience, but the research in this chapter still has some shortcomings: due to the differences in the detection instruments, the different backgrounds of the times, the different data sources, and the lack of specific ECG diagnostic experts to guide these factors in the reality, the basic research of this chapter needs to be further improved.

Further cooperation with hospital ECG experts in the later stage is expected, combined with computer method technology, to process more targeted ECG data and further improve the variant measurement system to form a standard model, and combined with pathological conditions; the corresponding quantitative evaluation criteria were studied.

It is necessary to specifically note here that the parameters selected in the experiments in this chapter are selected after a large number of experiments, and the selection is based on the integrity, usability, and stability of the image features in the visualization results.

\section{Acknowledgements}

Thanks to First People's Hospital of Yunnan Province for ECG data, Key project of Electric Information and Next Generation IT Technology of Yunnan (2018ZI002), National Science Foundation of China NSFC (61362014), and the Overseas Higherlevel Scholar Project of Yunnan for financial supports of the project.

\section{Author details}

Zhihui Hou ${ }^{1}$ and Jeffrey Zheng ${ }^{2 *}$

1 China Yangtze Power Co., Ltd., Hubei, China

2 School of Software, Yunnan University, Kunming, China

*Address all correspondence to: conjugatelogic@yahoo.com

\section{IntechOpen}

(C) 2019 The Author(s). Licensee IntechOpen. This chapter is distributed under the terms of the Creative Commons Attribution License (http://creativecommons.org/licenses/ by/3.0), which permits unrestricted use, distribution, and reproduction in any medium, provided the original work is properly cited. (cc) BY 


\section{References}

[1] Sabab SA, Munshi AR, Pritom AI, Shihabuzzaman. Cardiovascular disease prognosis using effective classification and feature selection technique. In: The International Conference on Medical Engineering, Health Informatics and Technology (MediTec); Dhaka, Bangladesh; 2016. pp. 1-6

[2] Subramanian B, Ramya K, Asokan R. Anatomizing electrocardiogram using fractal features and GUI based detection of $P$ and $T$ waves. In: International Conference on Wireless Communications, Signal Processing and Networking (WiSPNET); India; 2016. pp. 469-473

[3] Skowronski MD, Harris JG. Acoustic detection and classification of microchiroptera using machine learning: Lessons learned from automatic speech recognition. The Journal of the Acoustical Society of America. 2006;119:1817

[4] Özbay Y, Ceylan R, Karlik B. A fuzzy clustering neural network architecture for classification of ECG arrhythmias. Computers in Biology and Medicine. 2006;36(4):376-388

[5] Mishra AK, Raghav S. Local fractal dimension based ECG arrhythmia classification. Biomedical Signal Processing and Control. 2010;5(2):114-123

[6] Kutlu Y, Kuntalp D. Feature extraction for ECG heartbeats using higher order statistics of WPD coefficients. Computer Methods and Programs in Biomedicine. 2012;105(3):257-267

[7] Luz EJS, Schwartz WR, CámaraChávez G, Menott D. ECG-based heartbeat classification for arrhythmia detection: A survey. Computer Methods and Programs in Biomedicine. 2016;127:144-164

[8] Wang W, Wei Y, Guan N, Wang Y. The automatic detection and analysis of electrocardiogram based on Lorenz plot. In: IEEE International Conference on Robotics and Biomimetics (ROBIO); Zhuhai, China; 2015. pp. 644-649

[9] Chawla MPS. Segment classification of ECG data and construction of scatter plots using principal component analysis. Journal of Mechanics in Medicine and Biology. 2008;08(03):421-458

[10] Zheng ZJ, Maeder AJ. The Conjugate Classification of the Kernel Form of the Hexagonal Grid. Japan, Tokyo: Springer; 1992

[11] Zheng ZJ. Conjugate transformation of regular plan lattices for binary images [PhD thesis]. Monash University; 1994

[12] Zheng JZJ, Zheng CH. A framework to express variant and invariant functional spaces for binary logic. Frontiers of Electrical and Electronic Engineering in China. 2010;5:163

[13] Zheng JZJ, Zheng CH, Kunii TL. A Framework of Variant Logic Construction for Cellular Automata. In book: Cellular Automata Innovative Modelling for Science and Engineering, Edition: 1, Chapter: 16. InTech Press. 2011. DOI: 10.13140/2.1.4071.5208

[14] Zheng JZJ, Zheng CH. Variant measures and visualized statistical distributions. Acta Photonica Sinica. 2011;40:1397

[15] Zheng JZJ, Zheng CH. Variant simulation system using quaternion structures. Journal of Modern Optics. 2012;59:484

[16] Wang H, Zheng JZJ. 3D visual method of variant logic construction for random sequence. In: Proceedings of the 14th Australian Information Warfare and Security Conference; Perth; 2013 
[17] Zheng J, Zhang W, Luo J, Zhou W, Shen R. Variant map system to simulate complex properties of DNA interactions using binary sequences. Advances in Pure Mathematics. 2013;3:5

[18] Zheng J, Luo J, Zhou W. Pseudo DNA sequence generation of noncoding distributions using variant maps on cellular automata. Applied Mathematics. 2014;5:153

[19] Zheng J, Zhang W, Luo J, Zhou W, Liesaputra V. Variant map construction to detect symmetric properties of genomes on 2D distributions. Journal of Data Mining in Genomics \& Proteomics. 2014;5:1

[20] Heim D, Heim O, Zeng PA, Zheng J. Successful creation of regular patterns in variant maps from bat echolocation calls. Biological Systems: Open Access. 2016;5:166

[21] Ji Y, Zheng J, Xie Y, Shou T. Variant maps on normal and abnormal ECG data sequences. Biology and Medicine (Aligarh). 2016;8:336

[22] Hou Z, Zheng J. Mapping ECG signals on variant maps. In: Proceedings of the 2017 IEEE/ACM International Conference on Advances in Social Networks Analysis and Mining (ASONAM'17); 31 July-03 August 2017; Sydney, Australia: ACM; 2017

[23] Hou Z, Zheng J. Visualization of $P$ wave characteristics in ECG. In: Proceedings of the 2017 IEEE International Conference on Image and Signal Processing, Biomedical Engineering and Informatics (CISPBMEI'10); 14-16; IEEE; 2017

[24] Zheng J. Variant construction from theoretical foundation to applications. Springer-Nature. 2019. DOI: 10.1007/978-981-13-2282-2 
Section 2

\section{Clinical Applications of Electrocardiography}





\title{
Characteristics of Atrial Premature Beat ECG Signals on Variant Maps
}

\author{
Lihua Leng, Jeffery Zheng and Jing Zhang
}

\begin{abstract}
Premature heartbeat is also known as extrasystole. It means the foundation of sinus or ectopic heart rhythm, a certain pacemaker in the heart excitable earlier than the basic rhythm, cause the heart to be local or all happening prematurely remove pole. Premature atrial beats may lead to cardiomyopathy. The experimental data in this chapter are provided by Xishan People's Hospital of Wuxi city, including normal ECG signals and abnormal ECG signals (atrial premature beat). The two types of ECG data sequences are processed experimentally through the variant measurement model, and the differences in the variant maps are compared.
\end{abstract}

Keywords: atrial premature beat, ECG signal, data sequence, arrhythmia, variant maps

\section{Introduction}

Cardiovascular diseases are common diseases that seriously threaten human health [1], and the mortality rate caused by cardiovascular diseases continues to increase globally. ECG signal is the direct response of heart activity and the most effective way to analyze cardiovascular diseases. The object of this chapter is the atrial premature beat ECG signal in atrial arrhythmia. The experimental method is variant measurement model [2]. The provider of ECG data is Xishan People's Hospital of Wuxi city, Jiangsu Province China, and the later calibrator of ECG data is the First People's Hospital of Yunnan Province China. This chapter includes relevant background to introduce atrial premature beat [3], variant measurement model, experimental data, and variant maps of atrial premature beat signals. There is a significant difference between the variant maps of normal and atrial premature beat ECG signals obtained through variant measurement schemes.

This chapter uses the variant measurement model and the visualization method of feature clustering to study the variant measurement of ECG signal data sequences. ECG signal utilizes multivalued logic function and variant principle to form variant logic symbol on n-element ECG signal sequence and output variant maps and observe the difference between different ECG signal data. The obtained variant maps can analyze the ECG data from the macroscopic level and express the information that cannot be reflected by the traditional ECG in an intuitive way. The application of variant maps in ECG signal is an extension of the original ECG signal methods. In practical applications, it is expected that the variant maps can assist the application of traditional ECG in the medical field and help clinicians to diagnose the test results more conveniently. 


\section{Relevant background}

\subsection{Atrial premature beats}

Atrial arrhythmia is the most common arrhythmia clinically, which refers to a kind of arrhythmia caused by conduction obstruction when ectopic excitations are located in the atrium or conduction system passing through the atrium. It is mainly active arrhythmia, and atrial premature beat is the most common type of atrial arrhythmia. Atrial premature beats can be seen in normal healthy people, but in healthy people, there are few frequent atrial premature beats [4]. Atrial premature beats is more common in organic heart disease patients, Hyperthyroidism, Coronary heart disease, Cardiomyopathy patients if frequent atrial premature beat, is the precursor of Atrial fibrillation, Acute myocardial infarction can also occur frequent atrial premature beat. Figure 1 shows the comparison between normal ECG and atrial premature beat ECG.

The picture is the atrial premature beat ECG. Patient information: male, 46 years old, arrhythmia, mean heart rate $62 \mathrm{bpm}$, early occurrence of $\mathrm{P}^{\prime}$-QRS-T in limb leads, $160 \mathrm{~ms}$ in P'R interval, incomplete compensatory interval, such expression is a atrial premature beat.

\subsection{Variant measurement model}

In 2010, variant model [5] was proposed with the stability of cellular automata as an example; the effect of variant and non-variant functions on binary logic functional space was explained. In 2011, the conditional probability statistical distribution of the variant measurement structure [6] is discussed. By simulating the two-state quantum interaction system, the variant two-path simulation model was established. With the continuous development of variant construction, this model has been applied in many fields: coding and non-coding DNA [7] sequence detection, random sequence testing [8], classification of cellular automata [7], classification of echolocation in bats [9], ECG signals [10, 11], and variant construction [12].

\subsection{Variant logic}

The core theory of variant measurement model is variant logic, which is extended and evolved on the basis of classical logic. In the variant logic function, assuming that the input sequence is $\mathrm{N}$ long $\{\mathrm{X}, 0 \leq \mathrm{i}<\mathrm{N}\}$, the output data sequence $\{\mathrm{Y}, 0 \leq \mathrm{i}<\mathrm{N}\}$ is $\mathrm{N}-1$ according to the variant rule. On the basis of the variant logical function, the variant measurement model defines four basic variant logical symbols:

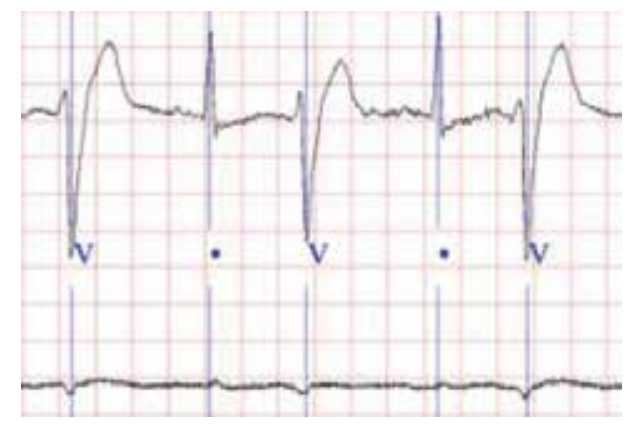

Figure 1.

Atrial premature beats ECG. 
$\{+,-, T, \perp\}$. The transformation rules of the four variant symbols are shown in Table 1. Therefore, in this chapter, $\mathrm{N}$ long ECG signal is converted into $\mathrm{N}-1$ long variant logical operator through variant logical function.

\subsection{Variant visualization}

The variant measurement model describes the measurement as the four meta symbols: $\{+,-, T, \perp\}$. The 16 subsets of meta symbol set are as follows: $\{\varnothing\},\{+\},\{-\},\{T\}$, $\{\perp\},\{+,-],\{+, \perp],\{+, T\},[-, T\},[-, \perp],[\perp, T\},\{+,-, T\},\{+,-, \perp],[+, T, \perp],\{-, T, \perp]$, and $[+,-, T, \perp]$.

The 16 subsets correspond to the 16 measures: $M_{0}, M_{1}, M_{2}, M_{3}, M_{4}, M_{5}, M_{6}, M_{7}, M_{8}, M_{9}, M_{10}, M_{11}, M_{12}, M_{13}, M_{14}, M_{15}$. Here, the statistical quantity of $\{\varnothing\}$ is defined as 0 and the corresponding definitions of 16 variant measures are shown in Table 2.

If any measure is selected as the value of $X$ in the variant maps and any measure is selected as the value of $\mathrm{Y}$ in the variant maps, there are a total of $16 * 16=256$ combinations of such two-dimensional maps, which are specifically shown in Table 3. In this chapter, the three-dimensional variant maps are also adopted, and the $\mathrm{Z}$-axis is added on the basis of the two-dimensional maps. The selection principle is the same as the selection method of $X$ and $Y$.

\subsection{ECG signal variant measurement structure}

Variant measurement structure is composed of three components: input, processing, and output. The input ECG signal is provided by Wuxi Xishan People's Hospital. The data processing module is the core module of variant measurement and consists of variant module, statistical measurement module, and visualization

\begin{tabular}{lccc}
\hline Conversion type & Variant sign & Statistical sign & Statistical total \\
\hline $0 \rightarrow 0$ & $\perp$ & $\mathrm{N}_{\perp}$ & $\mathrm{N}=\mathrm{N}_{\perp}+\mathrm{N}_{+}+\mathrm{N}_{-}+\mathrm{N}_{\mathrm{T}}$ \\
\hline $0 \rightarrow 1$ & + & $\mathrm{N}_{+}$ & \\
\hline $1 \rightarrow 0$ & - & $\mathrm{N}_{-}$ & \\
\hline $1 \rightarrow 1$ & $\mathrm{~T}$ & $\mathrm{~N}_{\mathrm{T}}$ & \\
\hline
\end{tabular}

Table 1.

Variant sign conversion rule.

\begin{tabular}{lccc}
\hline Sign subset & Variant measure & Sign subset & Variant measure \\
\hline$\{\varnothing\}$ & $\mathrm{M}_{0}=0$ & $\{-, T\}$ & $\mathrm{M}_{8}=\mathrm{N}_{.}+\mathrm{N}_{\mathrm{T}}$ \\
\hline$\{+\}$ & $\mathrm{M}_{1}=\mathrm{N}_{+}$ & $\{-, \perp\}$ & $\mathrm{M}_{9}=\mathrm{N}_{-}+\mathrm{N}_{\perp}$ \\
\hline$\{-\}$ & $\mathrm{M}_{2}=\mathrm{N}_{-}$ & $\{\perp, T\}$ & $\mathrm{M}_{10}=\mathrm{N}_{\perp}+\mathrm{N}_{\mathrm{T}}$ \\
\hline$\{T\}$ & $\mathrm{M}_{3}=\mathrm{N}_{\mathrm{T}}$ & $\{+, \cdot, T\}$ & $\mathrm{M}_{11}=\mathrm{N}_{+}+\mathrm{N}_{.}+\mathrm{N}_{\mathrm{T}}$ \\
\hline$\{\perp\}$ & $\mathrm{M}_{4}=\mathrm{N}_{\perp}$ & $\{+, \cdot, \perp\}$ & $\mathrm{M}_{12}=\mathrm{N}_{+}+\mathrm{N}_{+}+\mathrm{N}_{\perp}$ \\
\hline$\{+,-\}$ & $\mathrm{M}_{5}=\mathrm{N}_{+}+\mathrm{N}_{-}$ & $\{+, \mathrm{T}, \perp\}$ & $\mathrm{M}_{13}=\mathrm{N}_{+}+\mathrm{N}_{\mathrm{T}}+\mathrm{N}_{\perp}$ \\
\hline$\{+, \perp\}$ & $\mathrm{M}_{6}=\mathrm{N}_{+}+\mathrm{N}_{\perp}$ & {$[-, \mathrm{T}, \perp\}$} & $\mathrm{M}_{14}=\mathrm{N}_{-}+\mathrm{N}_{\mathrm{T}}+\mathrm{N}_{\perp}$ \\
\hline$\{+, \mathrm{T}\}$ & $\mathrm{M}_{7}=\mathrm{N}_{+}+\mathrm{N}_{\mathrm{T}}$ & {$[+,-, \mathrm{T}, \perp\}$} & $\mathrm{M}_{15}=\mathrm{N}_{+}+\mathrm{N}_{-}+\mathrm{N}_{\mathrm{T}}+\mathrm{N}_{\perp}$ \\
\hline
\end{tabular}

Table 2.

Definition of 16 variant measures. 


\begin{tabular}{cccccc}
\hline & $\mathrm{M}_{0}$ & $\mathrm{M}_{1}$ & $\cdots$ & $\mathrm{M}$ & $\mathrm{M}_{15}$ \\
\hline $\mathrm{M}_{15}$ & $\left(\mathrm{M}_{0}, \mathrm{M}_{15}\right)$ & $\left(\mathrm{M}_{1}, \mathrm{M}_{15}\right)$ & $\ldots$ & $\left(\mathrm{M}_{14}, \mathrm{M}_{15}\right)$ & $\left(\mathrm{M}_{15}, \mathrm{M}_{15}\right)$ \\
\hline $\mathrm{M}_{14}$ & $\left(\mathrm{M}_{0}, \mathrm{M}_{14}\right)$ & $\left(\mathrm{M}_{1}, \mathrm{M}_{14}\right)$ & $\ldots$ & $\left(\mathrm{M}_{14}, \mathrm{M}_{14}\right)$ & $\left(\mathrm{M}_{15}, \mathrm{M}_{14}\right)$ \\
\hline$\ldots$ & $\ldots$ & $\ldots$ & $\ldots$ & $\ldots$ & $\ldots$ \\
\hline $\mathrm{M}_{1}$ & $\left(\mathrm{M}_{0}, \mathrm{M}_{1}\right)$ & $\left(\mathrm{M}_{1}, \mathrm{M}_{1}\right)$ & $\ldots$ & $\left(\mathrm{M}_{14}, \mathrm{M}_{1}\right)$ & $\left(\mathrm{M}_{15}, \mathrm{M}_{1}\right)$ \\
\hline $\mathrm{M}_{0}$ & $\left(\mathrm{M}_{0}, \mathrm{M}_{0}\right)$ & $\left(\mathrm{M}_{1}, \mathrm{M}_{0}\right)$ & $\ldots$ & $\left(\mathrm{M}_{14}, \mathrm{M}_{0}\right)$ & $\left(\mathrm{M}_{15}, \mathrm{M}_{0}\right)$ \\
\hline
\end{tabular}

Table 3.

Combinations of visualization of 256 variant measures.

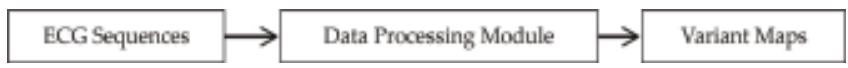

Figure 2.

Structure diagram of ECG sequence variant measurement.

module. The final output is variant maps. The structure of ECG signal variant measurement is shown in Figure 2.

\subsection{Data processing module}

The data processing module is divided into three parts: variant module, measurement statistics module, and visualization module. The variant module is the most important part of the variant measurement model. It is mainly responsible for transforming the original ECG signal sequence into the sequence of four basic variant logic symbols $\{+,-, T, \perp\}$ through mapping rules. The specific definitions of the parameters $X, T, L, V$ involved in the transformation process will be given in the core module. The measurement statistics module is mainly responsible for grouping the variant logical symbol $\{+,-, T, \perp\}$ sequence by setting a reasonable segment length $M$ according to total length of the sequence. After grouping, the $\{+,-, T, \perp\}$ sequence in each group is counted and denoted as $\left[\mathrm{N}_{+}, \mathrm{N}_{-}, \mathrm{N}_{\mathrm{T}}, \mathrm{N}_{\perp}\right]$. The visualization module is to generate the final variant maps, in which the variant maps can be two-dimensional map and three-dimensional map. The specific visualization process will be given in the chapter of the core module. The structure diagram of the data processing module is shown in Figure 3.

\subsection{Core module}

\subsubsection{Variant module}

The variant module is the most core module in the variant measurement model. It is mainly responsible for converting the obtained ECG signal sequence into four variant logical symbol $\{+,-, T, \perp\}$ sequences through variant logic. The transformation process involves parameters X, T, L, V, and the definition is shown in Eqs. (1)-(4).

$$
\begin{gathered}
\mathrm{X}=A_{i+1}-A_{i} \\
\mathrm{~T}=\frac{\sum_{i=1}^{N-1}\left|A_{i+1}-A_{i}\right|}{N}
\end{gathered}
$$




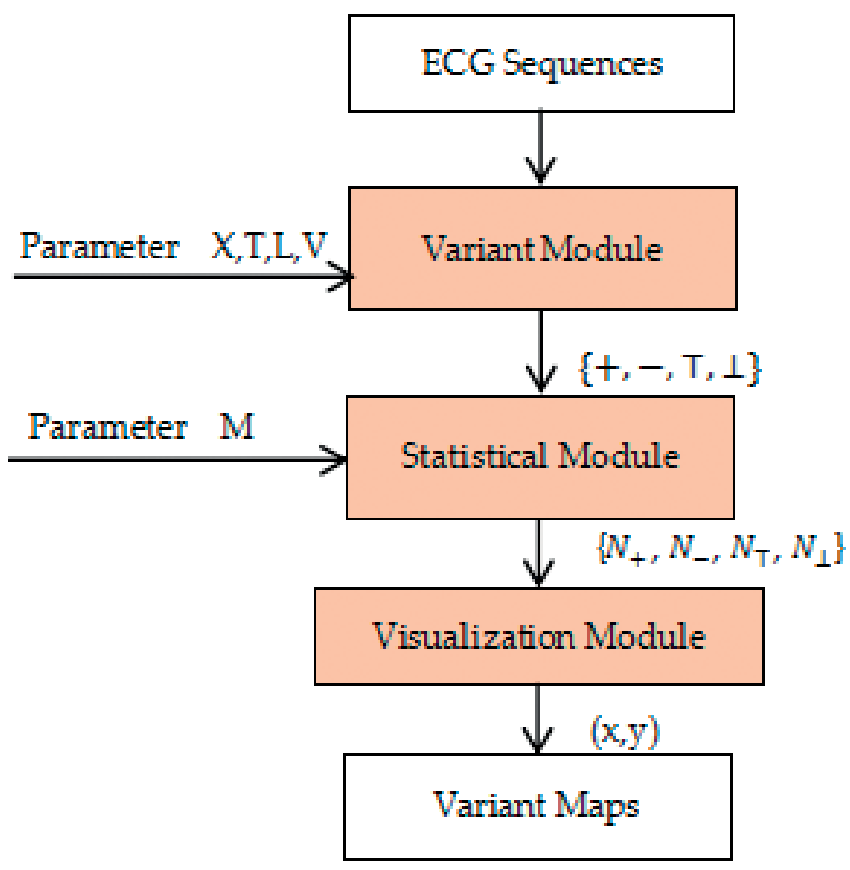

Figure 3.

Structure diagram of ECG data processing.

$$
\begin{gathered}
\mathrm{L}=\frac{A_{i}+A_{i+1}}{2} \\
\mathrm{~V}=\frac{\sum_{i=1}^{N-1} \frac{\left(A_{i}+A_{i+1}\right)}{2}}{N}
\end{gathered}
$$

Considering that the difference value can reflect the increase or decrease of adjacent ECG data, the difference value of the overall ECG data can reflect the fluctuation of the sequence; the mean value can reflect the level of adjacent ECG data, so the mean value of the overall ECG data can reflect the overall level of the sequence. In the process of data transformation, the variant module selects the difference, global difference, mean, and global mean as the measurement parameters. Using the parameters defined in Eqs. (1)-(4), the corresponding X, T, L, V parameter values can be calculated for the input n-long ECG signal sequence as the parameter support of the variant module. According to the mapping principle of Eq. (5), the original ECG sequence was mapped to $\{+,-, T, \perp\}$ sequence by setting parameters.

$$
\left\{\begin{array}{l}
\text { if } X>T \text { and } X>0: B_{i}=+ \\
\text { if } X>T \text { and } X \leq 0: B_{i}=- \\
\text { if } X \leq T \text { and } L>V: B_{i}=\top \\
\text { if } X \leq T \text { and } L \leq V: B_{i}=\perp
\end{array}\right.
$$

\subsubsection{Measurement statistics module}

As shown in Figure 3, for n-1 long variant logic symbol sequence, set the segment length parameter $M$, the number of groups is $\varsigma$, and then $S=(n-1) / M$. According to

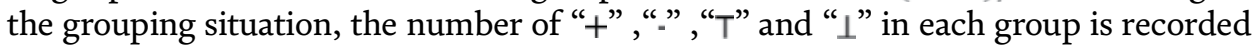


as " $\mathrm{N}_{+}$", "N.", " $\mathrm{N}_{\top}$ " and " $\mathrm{N}_{\perp}$ ", " $\mathrm{N}_{+}$" represents the number of statistics of the variant logic symbol "+" in this group. After statistical process, the whole output group is generated $\subseteq\left\{\mathrm{N}_{+}, \mathrm{N}, \mathrm{N}_{\mathrm{T}}, \mathrm{N}_{\perp}\right]$. For each set of statistical results, including $\left[\mathrm{N}_{+}, \mathrm{N}, \mathrm{N}_{\mathrm{T}}, \mathrm{N}_{\perp}\right]$ meet $0 \leq \mathrm{N}_{+}, \mathrm{N}_{-}, \mathrm{N}_{\mathrm{T}}, \mathrm{N}_{\perp} \leq \mathrm{M}, \mathrm{N}_{+}+\mathrm{N}_{\mathrm{.}}+\mathrm{N}_{\mathrm{T}}+\mathrm{N}_{\perp}=\mathrm{M}, \mathrm{n}-1$ long variant logical symbol sequence is converted into $S$ group statistical array $\left[\mathrm{N}_{+}, \mathrm{N}, \mathrm{N}_{\mathrm{T}}, \mathrm{N}_{\perp}\right]$.

\subsubsection{Visualization module}

The visualization module determines the selection of coordinates $(\mathrm{X}, \mathrm{Y})$ and generates a variant graph. According to the results obtained from the measurement statistics module and the definition of variant visualization, this chapter selects overall two-dimensional maps, two-dimensional combination maps, and threedimensional combination maps to display the visualization results. The overall twodimensional maps define normal and abnormal ECG signals as $4 * 4=16$ of 256 combinations, respectively. The two-dimensional combination maps is to form the scatter diagram of normal and abnormal ECG signals by the same mapping method, and it is easier to observe the differences between them on the same maps. Threedimensional combination maps are a combination method added on the basis of two-dimensional combination maps. In three-dimensional space, the characteristics between normal ECG signal and abnormal ECG signal are more abundant.

\section{Data sets}

The ECG data is provided by the people's hospital of Wuxi Xishan. The CB series ECG review analyzer is used to measure blood pressure of ECG data. Through the system of the patient's ECG information is stored in 18 data files, one of .dat files is stored in the patient's ECG data, by reviewing the ECG blood pressure of CB series data of ECG analysis system is read as shown in Figure 4.

As shown in Figure 4, the patient's ECG was collected with three-lead, and the heart rate data of $\mathrm{CH} 1$ lead was recorded at a sampling point of $1.5 \mathrm{~s}$ on average. The ECG signal was imported into the database for variant measurement experiment. This set of ECG data includes 105 patients. By analyzing the diagnosis results of each patient, this data set can be divided into two categories: normal and abnormal. Abnormal ECG data includes four types of symptoms: atrial premature beat, 64 cases; ventricular/atrial premature beats and T changes, 9 cases; premature ventricular/atrial beats and complete right bundle branch conduction block, 4 cases; atrial fibrillation and ST-T changes, 4 cases; and normal, 24 cases. In this chapter, atrial premature beat and normal ECG data were selected for variant measurement.

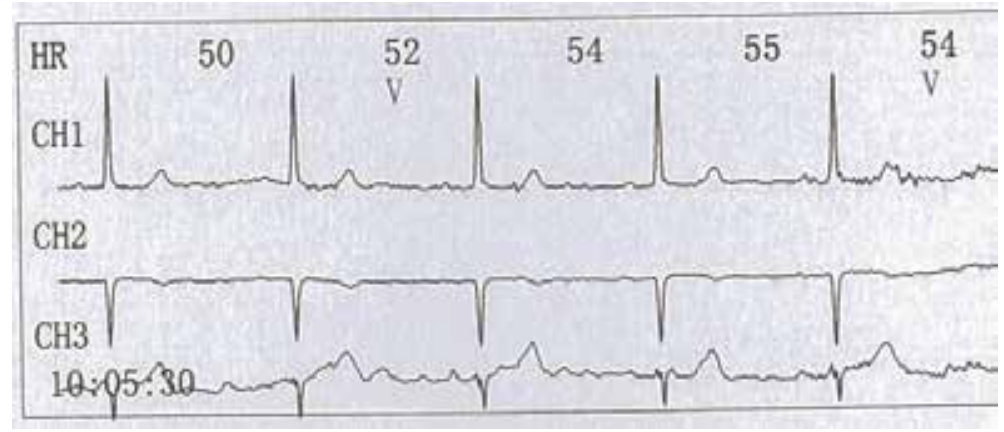

Figure 4 .

ECG data diagram of Xishan People's Hospital. 
Characteristics of Atrial Premature Beat ECG Signals on Variant Maps

DOI: http://dx.doi.org/10.5772/intechopen.83551

\section{Experimental results}

\subsection{Two-dimensional overall maps}

As shown in Figure 5, the red diagram on the left is a variant map of normal ECG signals; the blue on the right is a variant map of atrial premature ECG signals,

Normal ECG data of 2Dim $4 * 4$

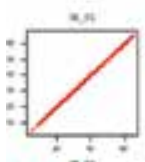

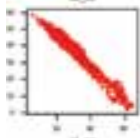

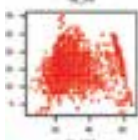

$\cdot \sqrt{\frac{2+2}{2}}$
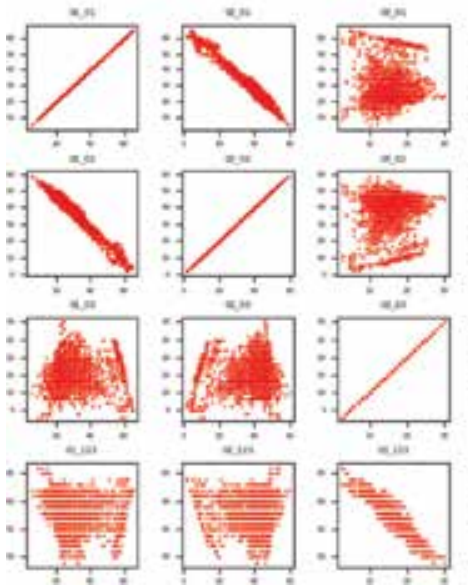
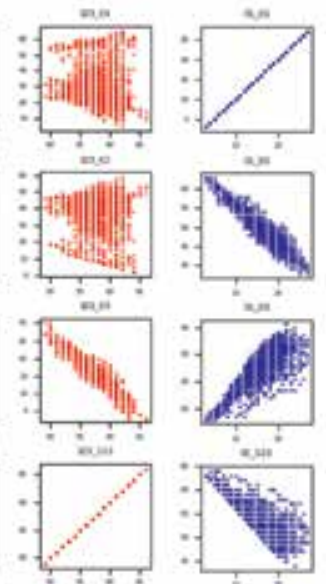

Atrial Premature Beat of 2Dim $4 * 4$
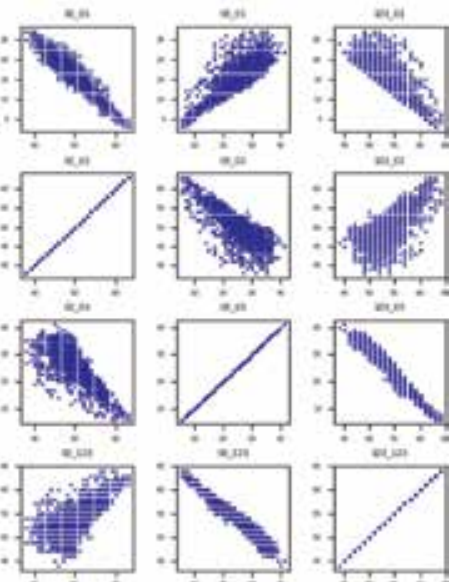

Figure 5 .

Overall two-dimensional maps of normal ECG data and atrial premature beat.
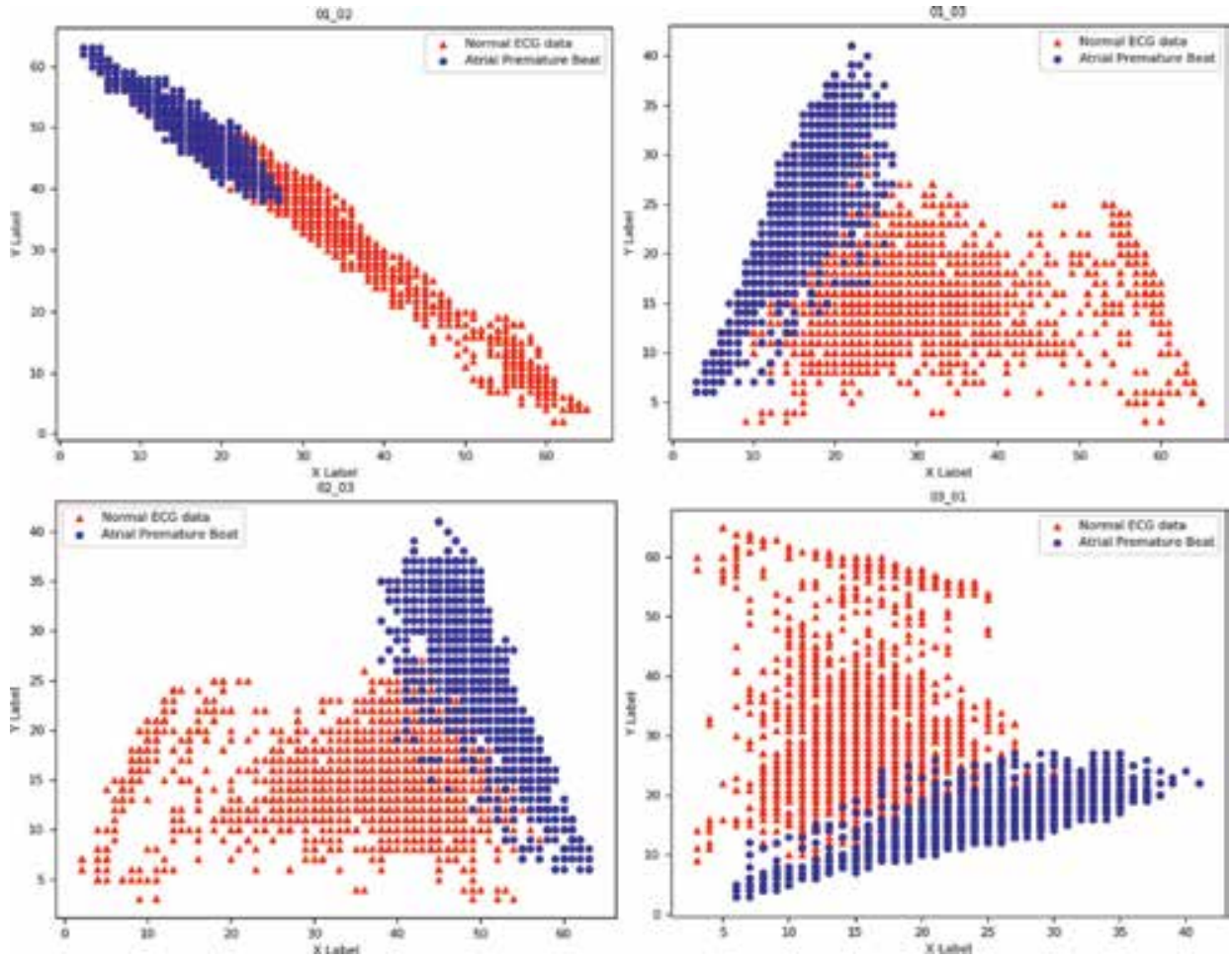

Figure 6.

Two-dimensional combination maps of normal ECG data and atrial premature beat. 
with the same mapping of $X=\left\{M_{5}, M_{6}, M_{7}, M_{14}\right\}, Y=\left\{M_{5}, M_{6}, M_{7}, M_{14}\right\}$ formed when the $4 * 4=16$ insets. At the top of each small map is the mapping mode corresponding to that diagram.

\subsection{Two-dimensional combination maps}

As shown in Figure 6, the two-dimensional combination maps put the results of the variant of the normal ECG signal and the atrial premature beat ECG signal in one picture, and the four combinations in Figure 6 are $\left(\mathrm{M}_{g^{\prime}} \mathrm{M}_{7}\right),\left(\mathrm{M}_{g^{\prime}} \mathrm{M}_{6}\right),\left(\mathrm{M}_{\sigma^{\prime}} \mathrm{M}_{5}\right)$, and $\left(\mathrm{M}_{7}, \mathrm{M}_{6}\right)$; the values of $\mathrm{X}, \mathrm{Y}$ are all limited to the sum of the two subsets.

As shown in Figure 7, the two-dimensional combination maps also put the results of the variant of the normal ECG signal and the atrial premature beat ECG signal in a single graph. The 12 combinations in Figure 7 are $\left(\mathrm{M}_{\mathrm{g}} \mathrm{M}_{14}\right),\left(\mathrm{M}_{\mathrm{G}} \mathrm{M}_{14}\right),\left(\mathrm{M}_{\mathrm{\gamma}}, \mathrm{M}_{14}\right),\left(\mathrm{M}_{\mathrm{E}} \mathrm{M}_{11}\right),\left(\mathrm{M}_{\mathrm{Q}} \mathrm{M}_{12}\right)$, and $\left(M_{\mathbb{E}^{\prime}} \mathrm{M}_{13}\right),\left(\mathrm{M}_{9} \mathrm{M}_{11}\right),\left(\mathrm{M}_{9}, \mathrm{M}_{12}\right),\left(\mathrm{M}_{\mathrm{g}} \mathrm{M}_{13}\right)\left(\mathrm{M}_{10}, \mathrm{M}_{11}\right),\left(\mathrm{M}_{10}, \mathrm{M}_{12}\right),\left(\mathrm{M}_{10}, \mathrm{M}_{13}\right)$, the value of $\mathrm{X}$ is limited to the sum of two subsets, and the value of $\mathrm{Y}$ is limited to the sum of the three subsets.
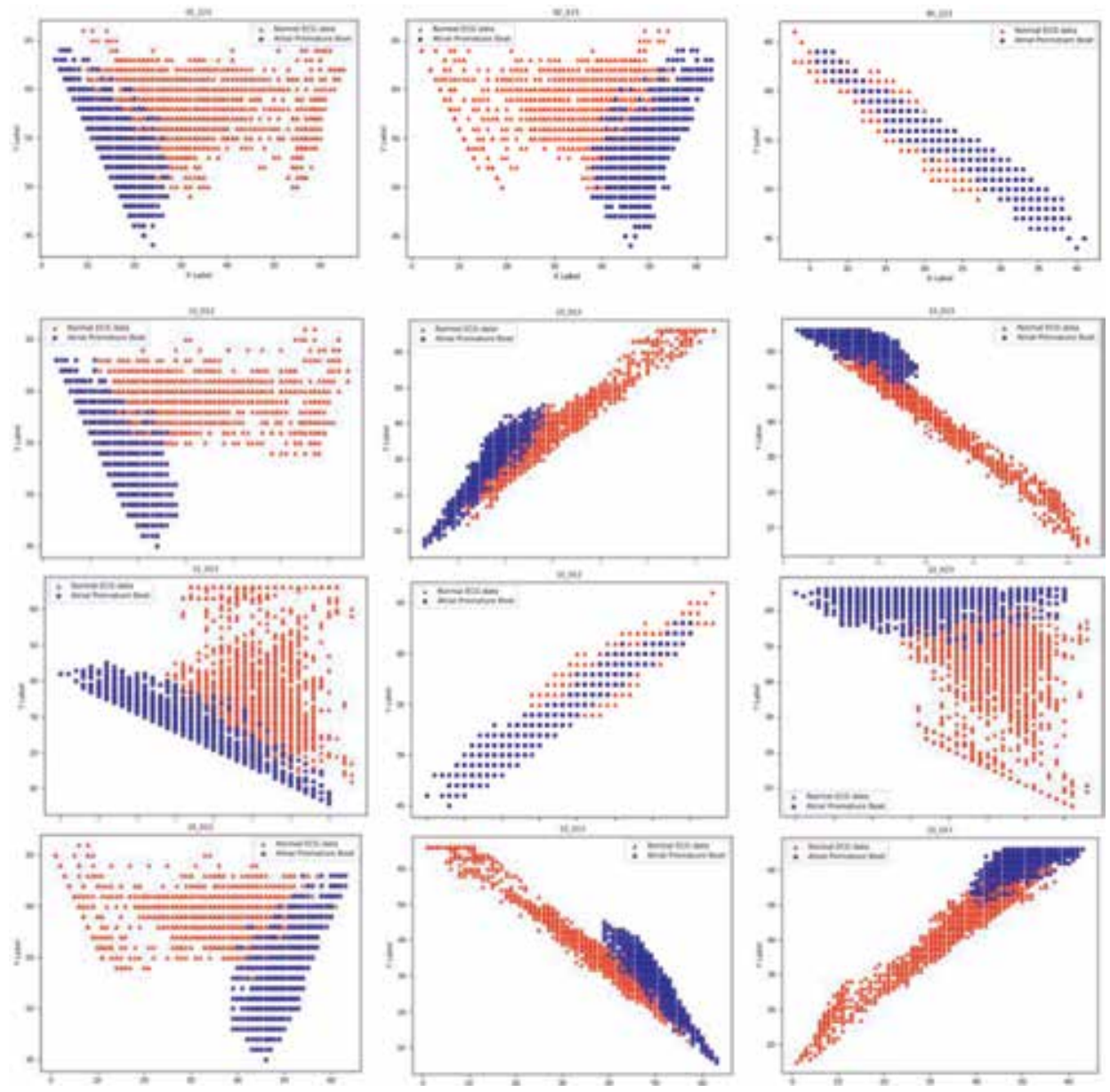

Figure 7.

Two-dimensional combination maps of normal ECG data and atrial premature beat. 


\subsection{Three-dimensional combination maps}

As shown in Figures 8-10, the three-dimensional combination maps are a combination of the normal ECG signal and the atrial premature beat ECG signal in the three-dimensional space. The mode of the $(\mathrm{X}, \mathrm{Y}, \mathrm{Z})$ combination in Figure 8 is $\left(M_{S} M_{E} M_{11}\right)$, the combination of $(X, Y, Z)$ in Figure 9 is $\left(M_{e} M_{g} M_{12}\right)$, and the combination of $(X, Y, Z)$ in Figure 10 is $\left(M_{2}, M_{10}, M_{13}\right)$; the values of $X$ and $Y$ are both defined as the sum of the two subsets, and the values of $\mathrm{Z}$ are all limited to the sum of the three subsets.
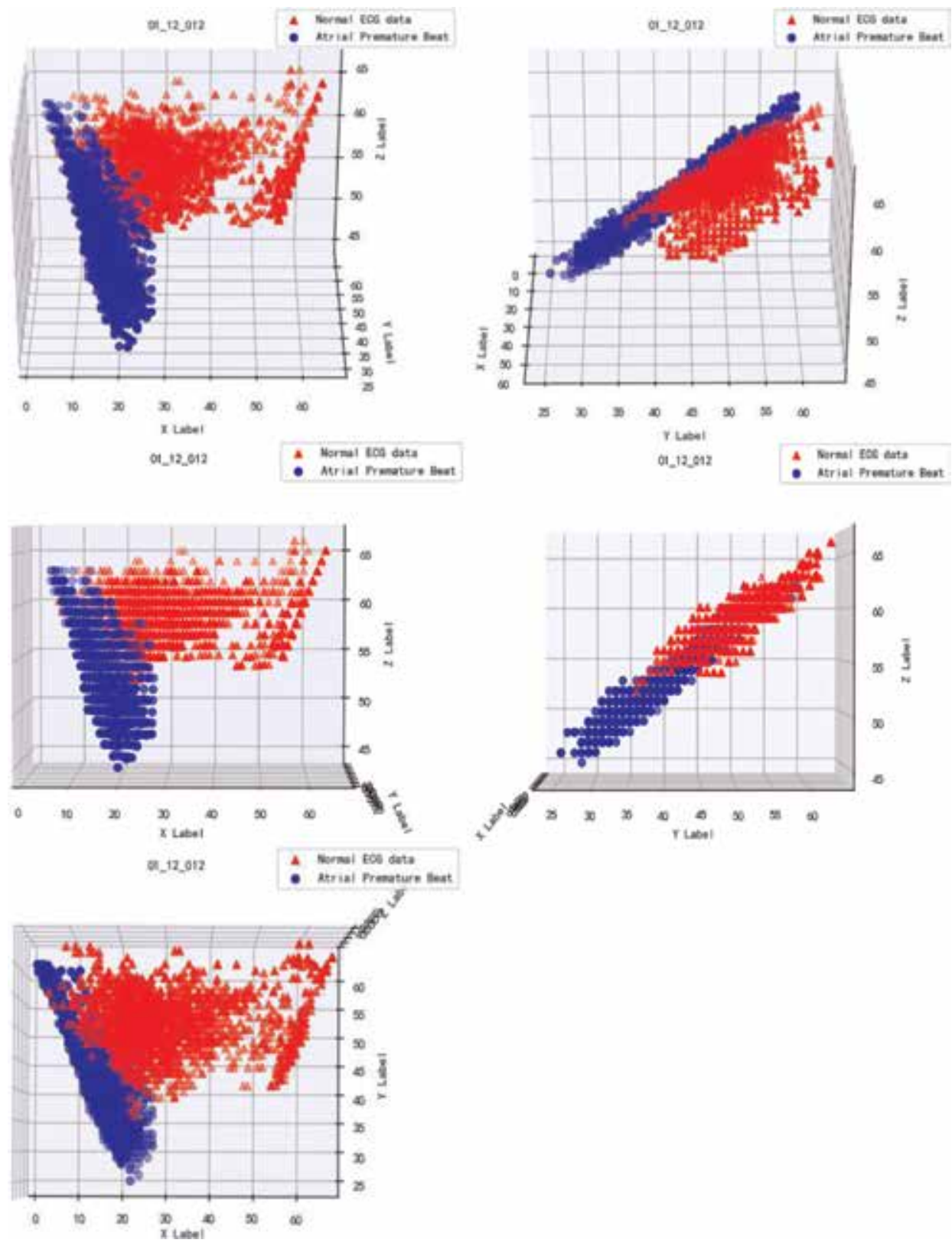

Figure 8.

Three-dimensional combination maps of normal ECG data and atrial premature beat. 

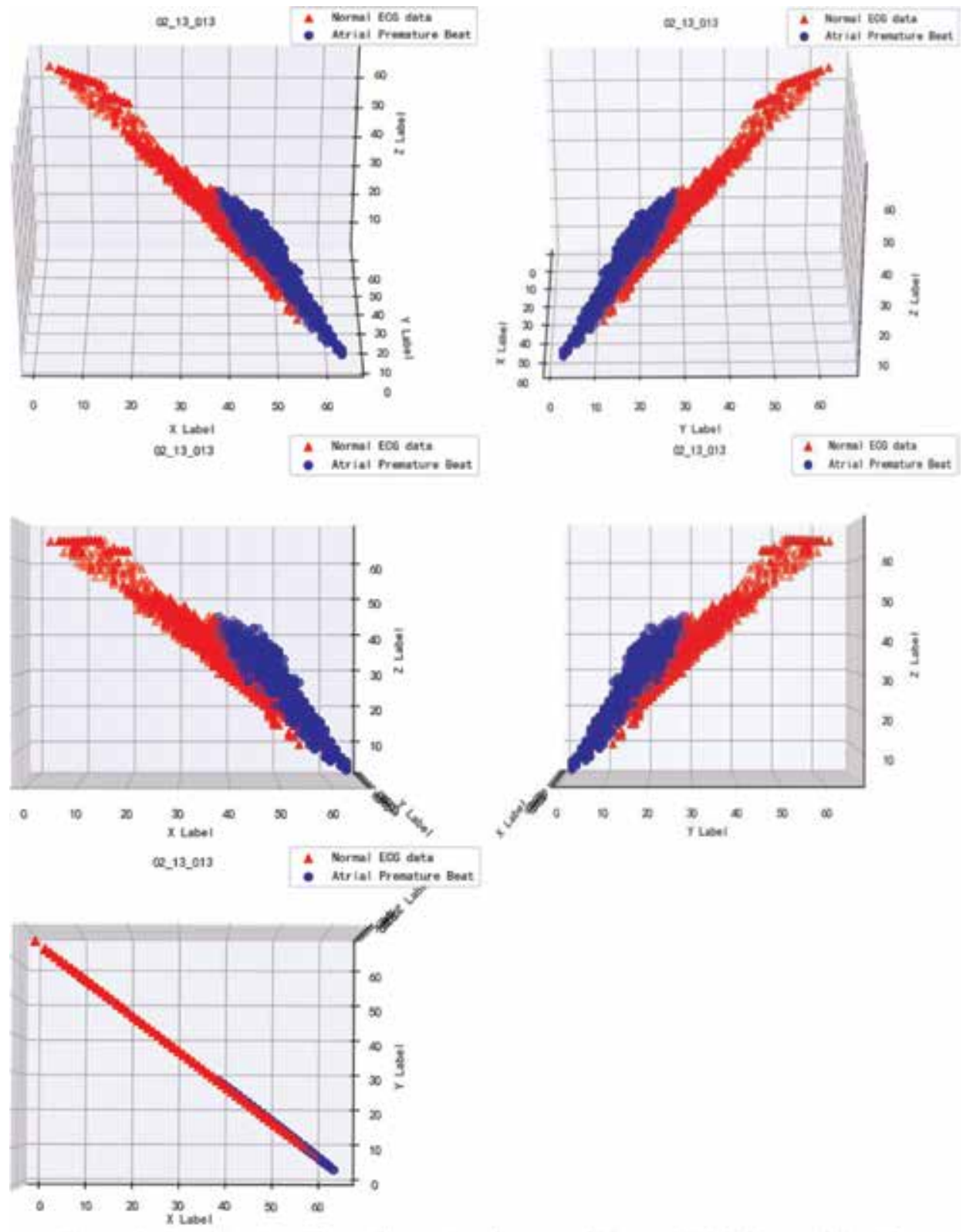

Figure 9.

Three-dimensional combination maps of normal ECG data and atrial premature beat.

\section{Results analysis}

Through the display of two-dimensional integral maps, two-dimensional combination maps, and three-dimensional combination maps, we can observe that:

The two-dimensional integral maps are to show the macro difference between the normal ECG signal and the atrial premature beat ECG signal through the variant experiment. From the two-dimensional whole figure, there are significant differences in the distribution shape and range between normal and abnormal ECG signals. The variant maps of the normal ECG signal show that the scattered points are disperse, even spreading the entire canvas, and the shape of the atrial premature 

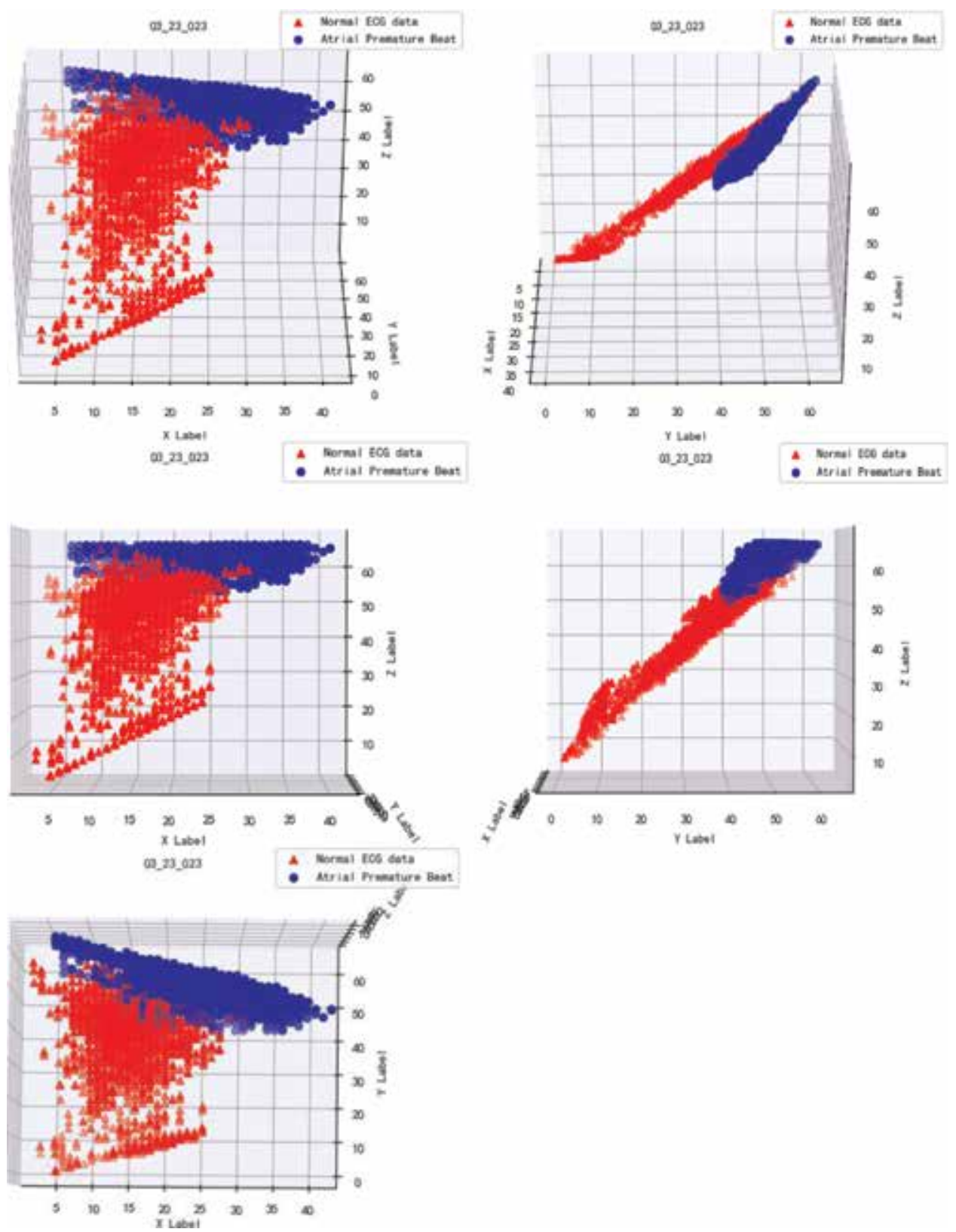

Figure 10.

Three-dimensional combination maps of normal ECG data and atrial premature beat.

beat ECG signal appears as an irregular cone. As the mapping mode changes, the shape of the variant maps also change, but the normal and abnormal ECG signals always exhibit different variant characteristics.

The two-dimensional combination map puts the variant maps of the two types of ECG signals on one canvas, and the difference between the normal and abnormal variant obtained by the same mapping method is more clear on one canvas. The mapping method selected in this chapter is more comprehensive, so the obtained variant map results are also universal.

The three-dimensional combination map is based on the two-dimensional combination maps subjoining the $\mathrm{Z}$-axis to form a spatial distribution display of the 
variant maps. The $(\mathrm{X}, \mathrm{Y}, \mathrm{Z})$ combination of the three-dimensional combination map selects $\left(\mathrm{M}_{g} \mathrm{M}_{\mathscr{g}}, \mathrm{M}_{11}\right),\left(\mathrm{M}_{\epsilon}, \mathrm{M}_{\mathrm{g}} \mathrm{M}_{12}\right)$, and $\left(\mathrm{M}_{\gamma}, \mathrm{M}_{10}, \mathrm{M}_{13}\right)$, and combines the two subsets and three subsets. The combination of the two is well integrated and is a more comprehensive illustration of the variant of normal and abnormal ECG signals. In the graphic display, five kinds of views are selected for each mapping method, which are $(\mathrm{X}, \mathrm{Y}, \mathrm{Z}),(\mathrm{Y}, \mathrm{X}, \mathrm{Z}),(\mathrm{X}, \mathrm{Z}),(\mathrm{Y}, \mathrm{Z})$, and $(\mathrm{X}, \mathrm{Y})$ as the screenshot of the main view. Through the results, we can see that there are also large differences in the three-dimensional spatial distribution of normal ECG signals and atrial premature beat ECG signals.

\section{Summary and outlook}

In this chapter, the variant measurement model is used to perform the variant experiment on the acquired batch ECG data. Finally, the experimental results of the variant measurement are displayed through three visualization methods. It is found through experiments that the variant measurement model can be well applied to the classification of normal ECG signals and atrial premature beat ECG signals.

In the future, we hope to get more abundant ECG data and can cooperate with the hospital to implement the application of the variant measurement model in the classification of ECG signals, so that it can assist the traditional ECG application in the clinical field.

\section{Acknowledgements}

Thanks to the Xishan People's Hospital of Wuxi City of Jiangsu Province for ECG datasets, to First People's Hospital of Yunnan Province for calibrator of ECG data, and to the Key Project of Electric Information and Next Generation IT Technology of Yunnan (2018ZI002), National Science Foundation of China NSFC (61362014), and the Overseas Higher Level Scholar Project of Yunnan for financial supports of the project. 


\section{Author details}

Lihua Leng ${ }^{1}$, Jeffery Zheng ${ }^{2 *}$ and Jing Zhang ${ }^{3}$

1 Technology and Product Management Department, Agricultural Bank of China, Yunnan Branch, Kunming, China

2 School of Software, Yunnan University, Kunming, China

3 Department of Cadre Health, Yunnan First People’s Hospital, Kunming, China

*Address all correspondence to: conjugatelogic@yahoo.com

\section{IntechOpen}

(C) 2019 The Author(s). Licensee IntechOpen. This chapter is distributed under the terms of the Creative Commons Attribution License (http://creativecommons.org/licenses/ by/3.0), which permits unrestricted use, distribution, and reproduction in any medium, provided the original work is properly cited. (c) BY 


\section{References}

[1] Thompson SC, Ting SA. Avoidance denial versus optimistic denial in reaction to the threat of future cardiovascular disease. Health Education \& Behavior the Official Publication of the Society for Public Health Education. 2012;39(5):620

[2] Zheng J, Zheng C. Variant measures and visualized statistical distributions. Acta Photonica Sinica. 2011;40(9): 1397-1404

[3] Mitchell LB. Atrial Premature Beats. The ECG. Springer. 2004:239-335

[4] Wallmann D, Tüller D, Wustmann $\mathrm{K}$, et al. Frequent atrial premature beats predict paroxysmal atrial fibrillation in stroke patients: An opportunity for a new diagnostic strategy. Stroke. 2007; 38(8):2292-2294

[5] Zheng J, Zheng C. A framework to express variant and invariant functional spaces for binary logic. Frontiers of Electrical and Electronic Engineering in China. 2010;5(2):163-172

[6] Zheng J, Zheng C, Kunii TL. A Framework of Variant Logic Construction for Cellular Automata, Cellular Automata-Innovative Modelling for Science and Engineering. Rijeka, Croatia: InTech Press; 2011

[7] Zheng J, Luo J, Zhou W. Pseudo DNA sequence generation of non-coding distributions using variant maps on cellular automata. Applied Mathematics. 2014;5(1):153-174

[8] Zheng J. Novel Pseudo Random Number Generation Using Varian Logic Framework. Australian Security

Congress. 2011:100-104

[9] Heim DM, Heim O, Zeng PA, Zheng J. Successful creation of regular patterns in variant maps from bat echolocation calls. Biological Systems: Open Access. 2016;5:166. DOI: 10.4172/2329-6577. 1000166

[10] Leng L, Zheng J. Mapping ECG signal sequences on variant maps. In: 2017 IEEE Trustcom/BigDataSE/ICESS; 2017. pp. 881-884. DOI: $10.1109 /$ Trustcom/BigDataSE/ICESS.2017.326

[11] Leng L, Zheng J. Visualization research of ECG sequence in sinus arrhythmia. Computer Science. 2016;43 (s2):183-185 (in Chinese)

[12] Zheng J. Variant Construction from Theoretical Foundation to Applications. Springer-Nature. 2019. DOI: 10.1007/ 978-981-13-2282-2 


\title{
Ventricular Tachycardia and Heart Failure
}

\author{
Hakan Altay
}

\begin{abstract}
Ventricular tachycardia (VT) is a common arrhythmia seen in patients with heart failure (HF) and is now seen more frequently as these patients survive longer with modern therapies. In patients with HF, half of the deaths are sudden due to life-threatening ventricular arrhythmias, including VT. Although disease modifying drugs, such as beta blockers, mineralocorticoid drugs, and angiotensin receptor neprilysin inhibitors, prevent the occurrence of VT to some extent, the mainstay of therapy is the antiarrhythmic drug therapy, implantable cardioverter-defibrillator (ICD) implantation, and traditional radiofrequency catheter ablation. Autonomic nerve system modulation and stereotactic body radiation therapy have emerged as novel techniques for the management of refractory VT cases. Patients with refractory VT and repetitive ICD shocks should be further evaluated regarding the candidacy for left ventricular assist device and transplantation.
\end{abstract}

Keywords: ventricular tachycardia, heart failure, antiarrhythmic therapy, implantable-cardioverter defibrillator, ablation

\section{Introduction}

Ventricular tachycardia (VT) is common in patients with heart failure (HF). The presence and severity of VT increase as the severity of HF increases. Larger infarcts with greater left ventricle (LV) systolic dysfunction are more likely to be associated with VT. VT forms one of the most common electrical mechanisms responsible for sudden cardiac death (SCD) in HF. Patients with LV systolic dysfunction who develop VT are at increased risk of SCD from subsequent VT or ventricular fibrillation [1].

Patients with VT and HF may present either with cardiac arrest to the emergency department or with palpitations, syncope, chest pain, or ICD shocks to cardiology outpatient clinics, varying according to the hemodynamic stability of VT. Both non-sustained VT (VT duration < $30 \mathrm{sec}$ ) and sustained VT (VT duration $>30 \mathrm{sec}$ ) in patients with HF are associated with significant morbidity and mortality. VT storm (three or more separate episodes of sustained VT requiring intervention (such as ICD shock or ATP) within 24 hours) is the most troublesome condition related with VT and HF.

Although half of the patients with HF have preserved ejection fraction and SCD is also a common issue in these patients, there is no proved treatment either by ICD or drugs [2]. Because of this, VT and HF will be discussed in the context of HF with reduced ejection fraction (HFREF). 


\section{Epidemiology}

Ventricular tachycardia is common in patients with HF, with up to $20 \%$ of patients developing VT in 5 years after an ICD was placed [3]. In patients with HF, SCD occurs 6-9 times more often than the general population [4]. The most studied and proven predictor of ventricular tachyarrhythmia and SCD is left ventricle ejection fraction (LVEF) [5]. It has been shown that once the LVEF recovered, the incidence of ventricular tachyarrhythmia decreases [6].

The threshold of LVEF $<35 \%$ represents an accepted threshold at which SCD risk is increased and primary prevention is indicated. Several other risk predictors of VT, such as non-sustained VT, programmed ventricular stimulation on electrophysiological study (EPS), microvolt T-wave alternans, late potentials on signalaveraged electrocardiogram, absence of heart rate variability, QT wave dispersion, baroreflex sensitivity, and heart rate turbulence have been proposed for patients with HF. However, none of these predictors has influenced the clinical practice.

\section{Pathophysiology}

There are multiple mechanisms that play a role in the occurrence of VT in patients with HF (Table 1). Adverse remodeling and progressive fibrosis occur in the ventricle following myocardial infarction (MI) or in association with nonischemic cardiomyopathy. These structural alterations as well as the ion channel changes form the essential substrate for the induction of VT [7].

The most common mechanism for VT is electrical reentry within and around patches of heterogenous myocardial fibrosis, most commonly occurring in areas of scar post-myocardial infarction or non-ischemic cardiomyopathy [8]. The scarrelated VT is typically monomorphic with single QRS morphology. Induction of monomorphic VT during EPS predicts patients who have the risk of spontaneous VT. Polymorphic VT is defined as a continually changing QRS morphology, often associated with acute ischemia, drugs which lead to QT prolongation or electrolyte imbalance.

Increased sympathetic nervous system (SNS) activation is another trigger for induction of VT. SNS activation, via beta-adrenoreceptors activates ryanodine receptor on the sarcoplasmic reticulum inside the cardiomyocytes leading to efflux of calcium and increase of intracellular concentration which is a trigger for VT. This is the rationale under the effect of beta blockers in suppressing VT, as well as SCD in HF patients.

\begin{tabular}{l}
\hline Mechanisms \\
\hline Positive remodeling and fibrosis \\
\hline Myocardial scar \\
\hline Electrolyte abnormalities \\
\hline Increased sympathetic tone \\
\hline Ischemia \\
\hline Abnormal calcium handling \\
\hline Delayed after depolarization \\
\hline Drugs \\
\hline
\end{tabular}

Table 1.

Mechanisms of VT occurrence in patients with heart failure. 
VT occurring within 24-48 hours of acute MI is called primary VT, and acute ischemia is considered to be the transient or correctable cause of VT in this case. Revascularization is the primary management form of primary VT. VT occurring after 48 hours of acute MI is called secondary VT, which is associated with worse clinical outcomes.

Increased diastolic calcium levels, early and delayed after depolarizations, and some of the drugs also cause VT. Antiarrhythmic drugs are the foremost drugs causing VT. Digoxin that is commonly used in the management of HF is an arrhythmogenic drug. Dobutamine treatment for acute decompensated HF has also been associated with VT [9]. Because of this, patients should be continuously monitorized during treatment with dobutamine. VT can also present as a complication of left ventricular assist device in an advanced HF patient. Most of these types of VT occur perioperatively [10]. It is important to find out the definite mechanism of VT in order to implement the best effective treatment. In patients with sarcoidosis, for example, VT can occur as a result of inflammation, scar or both. If VT is thought to be due to inflammation, best treatment is antiarrhythmic drug and immunosuppressive, whereas if VT is of scar related, best treatment is antiarrhythmic drug and catheter ablation [11].

\section{Management}

Management of VT in heart failure poses a great challenge to cardiologists since antiarrhythmic drugs are limited by incomplete efficacy and unfavorable adverse effect profile, ICD is complex and expensive and may affect the quality of life adversely because of inappropriate shocks, and invasive catheter ablation owns the risk of complication and recurrence. Therefore, multidisciplinary team approach including electrophysiologists, heart failure specialists, general cardiologists, intensivists, and cardiovascular surgeon should be used to tackle such a difficult disease.

VT is a life-threatening condition and needs urgent management. Acute management of VT in HF patients depends on the hemodynamic stability of the patient. In hemodynamically unstable VT, the priority is electrical direct current cardioversion [12]. If the patient is hemodynamically stable, a trial of antiarrhythmic treatment should be applied. Intravenous amiodarone is the most effective and safe antiarrhythmic treatment in this case [12].

Slow VT $(<150$ beats/minute) may be tolerated in the short term (Figure 1). However, slow VT in the presence of poor ventricular function may cause hemodynamic compromise in the long term. It is important to closely monitor the patient while administering antiarrhythmic therapy. If the antiarrhythmic therapy does not cardiovert the patient, shock should be applied as early as possible since sustained VT can compromise hemodynamic status of the patient with left ventricular dysfunction in due course. The initial approach to the management of VT should include evaluation for correctable causes of VT (e.g., electrolyte abnormalities and ischemia). Electrolyte abnormalities, particularly hypokalemia and hypomagnesemia which are known to facilitate VT in HF patients should be corrected. Potassium and magnesium levels should be kept $>4 \mathrm{meq} / \mathrm{l}$ and $>2 \mathrm{meq} / \mathrm{l}$, respectively. Agents, for example, digoxin, that may induce arrhythmia should be withheld.

For chronic management of VT, optimization of guideline-directed medical treatment is very important especially in patients with HFREF. Until recently, these treatments consisted of angiotensin converting enzyme inhibitors (ACEis) or angiotensin receptor blockers (ARBs), beta blockers (BBs), and mineralocorticoid receptor antagonists (MRAs). Of these guideline-directed medical treatments, $\mathrm{BB}$ and MRA have been proved to prevent sudden cardiac death $[13,14]$. These drugs have the ability to improve reverse modeling which reduces VT. BBs are the 


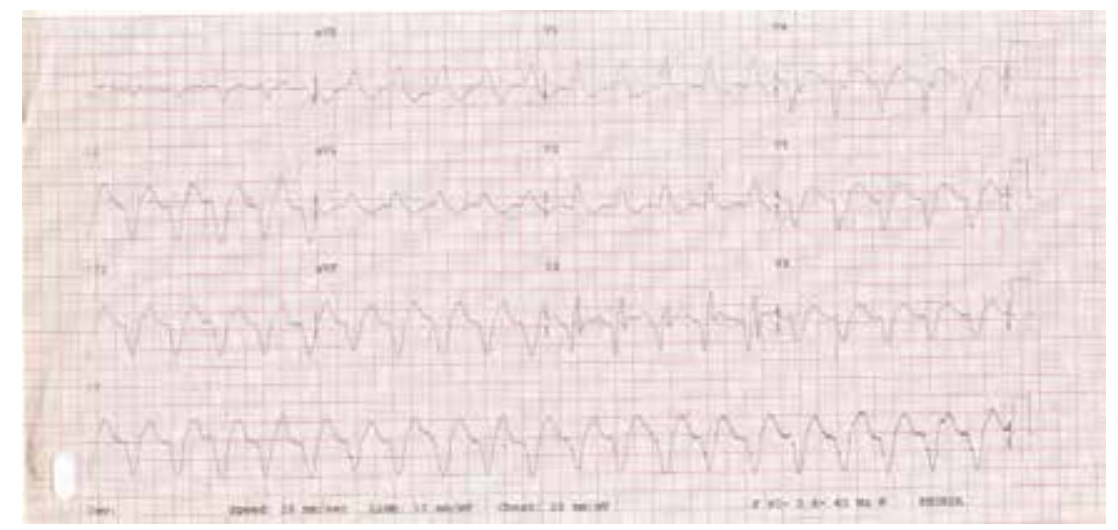

Figure 1.

Slow VT at a rate of approximately 125/bpm in a patient on high dose of beta blocker and amiodarone.

first-line therapy for the management of VT in HF patients. In MADIT-II trial (the Multicenter Automatic Defibrillator Implantation Trial II), patients with ICD treated with the highest dose of $\mathrm{BB}$ experienced less ICD treatment compared to patients not taking BB [15].

A meta-analysis compared medical treatment with ICD preventing SCD in patients with $\mathrm{HF}$ and left ventricular systolic dysfunction. MRAs were found to be the most effective drug when added to ACEi and BB, in preventing SCD [16]. Zannad et al. also showed that MRAs were equally effective in preventing SCD in patients with ICD as without ICD [17].

A newly emerged drug in HFREF, angiotensin receptor neprilysin inhibitor (ARNi), was compared with enalapril in PARADIGM-HF trial (prospective comparison of angiotensin neprilysin inhibitor (ARNI) with ACE-i to determine impact on global morbidity and mortality in heart failure) [18]. ARNi was shown to be superior in reducing cardiovascular death and hospitalization compared to enalapril. ARNi also reduced SCD by $20 \%$ compared to enalapril. European Society of Cardiology $2016 \mathrm{HF}$ guideline has made a class 1 recommendation regarding the use of BB, MRA, and ARNi in patients with HFREF and VT [19].

Optimum use of guideline-directed medical treatment prevents development of VT to some extent. If the patient continues to be at risk of VT because of low ejection fraction, non-sustained or sustained VT, antiarrhythmic drugs, ICD implantation, and VT ablation are the subsequent treatment options for chronic management of VT. General use of antiarrhythmic drugs in HF is not recommended for VT since these drugs, except amiodarone, have been shown to increase mortality in patients with HF due to proarrhythmic or negative inotropic effects.

Notorious CAST trial (Cardiac Arrhythmia Suppression Trial) showed that class $1 \mathrm{C}$ agents, encainide, and flecainide increases mortality and non-fatal cardiac arrest when used to suppress VT post-myocardial infarction [20]. CAST trial was planned to answer the question of whether suppressing ventricular premature beats (VPB) also aid in reducing mortality. Patients who had myocardial infarction within the preceding 2 years and $>6$ VPBs on holter recording were enrolled. Those who had MI within 90 days were required to have $\mathrm{EF}<55 \%$, and those who had MI after this period were required to have $\mathrm{EF}<40 \%$. Patients were randomly assigned to class $1 \mathrm{C}$ agents (encainide, flecainide, or moricizine). Patients whose PVBs were suppressed were allocated to the treatment with one of the class $1 \mathrm{C}$ agent or placebo. The trial was prematurely stopped based on the in-term analysis that showed that encainide and flecainide used to suppress VPBs increased the mortality by 2.5 times. It is likely that mortality excess can be attributed to the proarrhythmic effects of encainide and flecainide. 
Amiodarone is the sole agent that can be used safely for suppression of VT in HF patients. Amiodarone has been studied extensively in patients with left ventricular dysfunction. Its efficacy for decreasing mortality in patients with VT and LV dysfunction has not been shown in SCD-HeFT trial (the Sudden Cardiac Death in Heart Failure Trial) [21]. However, a meta-analysis including 8522 patients post-myocardial infarction or with systolic HF showed that amiodarone reduced SCD and cardiovascular mortality [22]. Its safety, unlike class 1 antiarrhythmic agents, has been confirmed in this patient population. In patients with more severe HF, amiodarone use is associated with adverse prognosis [21]. Amiodarone cannot be used for a long period of time because it is associated with multiple side effects, primarily affecting thyroid, lung, liver, skin, and eye [23]. Therefore, regular monitoring of lung, liver, and thyroid function is required. Due to these side effects, discontinuation rates of amiodarone have been noted to be high [22].

Sotalol, a group III antiarrhythmic drug, with BB properties, is highly effective in suppressing VT but it is contraindicated in HF patients since increased mortality was demonstrated when D-sotalol was used in patients with left ventricular dysfunction after myocardial infarction in SWORD trial [24]. Dofetilide, another class III antiarrhythmic drug, failed to reduce arrhythmic death in patients with HF [25]. If VT occurs despite amiodarone therapy, mexiletine can be used as an adjunct to amiodarone.

Electrophysiologic study was once used for identification of successful antiarrhythmic therapy and also the patients who require other advanced therapies. Patients were given certain antiarrhythmic drugs after VT was induced at programmed stimulation. Patients on chronic oral antiarrhythmic drug were then assessed whether VT could be induced again [26].

Of the therapies currently available to manage VT, ICD is by far the most effective one and has the best supported safety and efficacy data from the trials and registries. An ICD has two important components: an ICD generator and a lead for sensing, pacing, and shock delivery (Figure 2). ICD improves the survival of patients who had VT and syncope, patients who had VT and LVEF $<40 \%$, and hemodynamic compromise [27]. ICD has been shown to prevent sudden cardiac death prophylactically in patents with LVEF $<35 \%$ resulting both from ischemic or non-ischemic cardiomyopathy $[21,28,29]$. The important issue in these primary prevention groups is that they should have already received guideline-directed medical treatment for at least 3 months before ICD implantation is planned. Electrophysiologic study is no longer a required procedure before planning ICD for primary prevention.

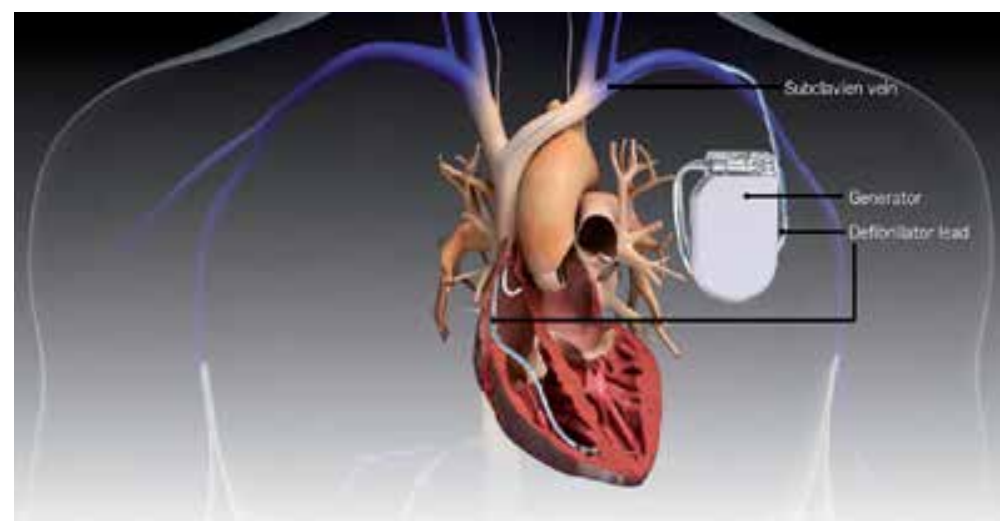

Figure 2.

A schematic representation of an intracardiac defibrillator implanted to right ventricle of heart failure patient. 
ICD has antitachycardia pacing (ATP) treatment in addition to defibrillator shock and also programs which can discriminate supraventricular tachycardia from VT which aids to minimize inappropriate shocks. ATP consists of one or more sequence of pacing stimuli, generally expressed as a percentage of tachycardia cycle length for a given RR interval. In case of burst ATP, pacing stimuli is delivered at constant coupling intervals, whereas ramp ATP consists of pacing stimuli with decrement coupling interval (Figure 3). Once VT is confirmed, first therapy in the form of ATP was given, and if ATP does not work, then shock is delivered. Generally ICD's VT detection zone is programmed to $>167$ beats $/ \mathrm{min}$ and ventricular fibrillation detection zone to $>185-200$ beats/min. Antiarrhythmic drugs commonly prolong VT cycle length and hence cause slow VT, a condition which may require to lower the detection zone for VT (Figure 1). In secondary prevention patients with HF, the programming of detection zone depends on the cycle length of the VT occurred. Generally, the detection zone is programmed $20 \mathrm{bpm}$ slower than the rate of the VT occurred before. ATP for faster VT (188-250 bpm) may also be programmed with the aim for reducing shocks. ICD shocks are related with poor prognosis and quality of life. For this reason, every effort should be made to reduce shocks. It was shown that reducing defibrillator shocks was associated with increased survival [30].

Cardiac resynchronization therapy (CRT) is also an important milestone in the management of moderate to severe HF patients with prolonged QRS duration (>150 msn and LBBB morphology). CRT without defibrillator (CRT-P) can prevent SCD by improving reverse remodeling. CARE-HF Trial (Cardiac Resynchronization-Heart Failure) showed that CRT-P prevents SCD by $46 \%$ in the long term follow-up [31]. Although CRT was shown to reduce new onset VT, it had no effect on recurrent VTs [32].

In patients with HF who are refractory to antiarrhythmic therapy, radiofrequency catheter ablation has emerged as an important therapeutic option. The success rate of this technique varies according to the type of cardiomyopathy. The American Heart Association(AHA)/the Heart Rhythm Society (HRS) recommends the use of VT ablation in patients with prior myocardial infarction and recurrent VT, unresponsive or intolerant to antiarrhythmic treatment [8]. Electrophysiologic study (EPS) with programmed electrical stimulation is recommended before ablation in case of sustained monomorphic VT in patients with prior MI [33]. Catheter ablation can be effective, but acute complications and long-term VT recurrence risk necessitating repeat ablation should be recognized. And worth notifying, procedure
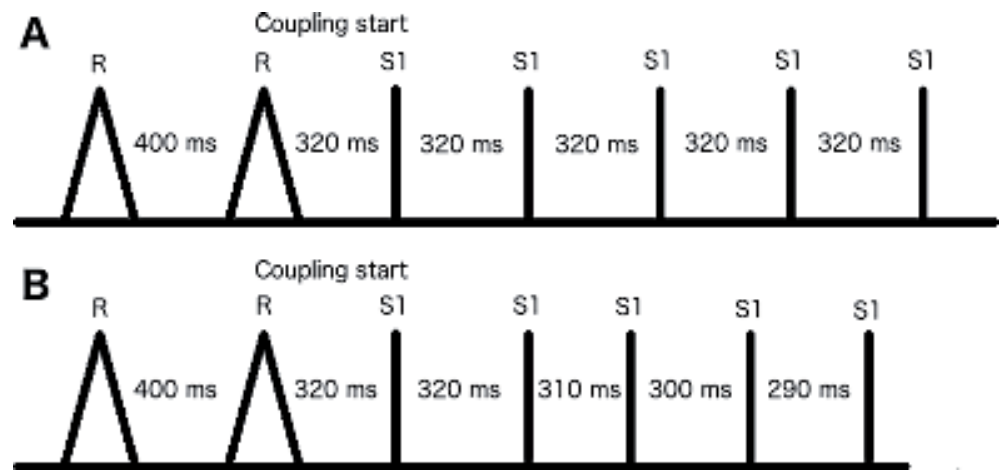

Figure 3.

Antitachycardia pacing (ATP) therapy of intracardiac defibrillator. (A) Burst ATP; pacing stimuli at lower than VT and constant coupling interval. (B) Ramp ATP; pacing stimuli starting with lower than VTcycle length and coupling intervals decreasing at each stimuli. 
of ablation lasts for long hours with extended recovery times. If VT remains refractory to catheter ablation, repeat ablation may be tried. If the first ablation was done by endocardial mapping, repeat ablations may be carried by epicardial mapping. Surgical ablation is indicated in patients with VT refractory to antiarrhythmic drugs whose catheter ablation has failed [12]. It was shown that surgical cryoablation guided by endocardial and epicardial mapping along with aneurysmectomy when indicated was a successful way of terminating VT in patients who underwent bypass operation [34].

Due to multiple mechanisms of VT in idiopathic dilated cardiomyopathy, the success rate of catheter ablation is less than in ischemic cardiomyopathy. It has been shown that ablation in this type of cardiomyopathy results in higher recurrence rate of VT than ischemic cardiomyopathy [35]. Catheter ablation of VT in dilated cardiomyopathy should only be done in patients with clear mechanism of VT (e.g., bundle branch reentry) only in experienced centers. Despite these shortcomings, successful VT ablation in non-ischemic dilated cardiomyopathy has increased. Predictors of recurrence after VT ablation in non-ischemic dilated cardiomyopathy were found to be inducibility of sustained VT in the programmed stimulation study, poor systolic function $(\mathrm{EF}<35 \%)$, and delayed intervention time [36].

Worth mentioning, there are some types of VTs occurring in the structurally normal heart, termed idiopathic VT. Idiopathic VT is further categorized according to the anatomic location in the heart. Most of them originate from the right ventricular outflow tract (RVOT) and have left bundle branch block (LBBB) pattern on the electrocardiogram. The second most common idiopathic VT originating from the conduction system is termed as fascicular VT. The other idiopathic VT originates from the mitral or tricuspid annulus and termed as annular VT. The clinical course of idiopathic VT is usually benign; however, if they occur in the form of incessant VT, they may cause LV systolic dysfunction, termed as arrhythmia-induced cardiomyopathy (AIC). It is important to differentiate AIC from non-ischemic dilated cardiomyopathy because RF ablation is the first line treatment and curative in the former [8]. VT originating from left ventricular outflow tract (LVOT) is rare compared to VT originating from RVOT. Some form of VTs originating from LVOT cannot be ablated by using conventional approach. This unique type of VT with LBBB inferior axis and early precordial transition can successfully be ablated from the aortic root, using either the left or non-coronary aortic sinus of valsalva [37]. VTs can also originate from papillary muscle of left or right ventricle. Ablation of papillary muscle VT is difficult compared to other idiopathic VTs. However, there is a case report showing successful ablation of incessant VT originating from posterior papillary muscle of right ventricle [38]. EPS is highly recommended before ablation of VT in structurally normal hearts which are suspected to be originated from RVOT, LVOT, aortic cusps, and epicardial VT [33]. EPS has also a role in case of sustained monomorphic VT in patients with arrhythmogenic right ventricular dysplasia (ARVD). It was shown that inducibility of sustained monomorphic VT during EPS highly predicts SCD, heart transplantation, VT with hemodynamic compromise, or syncope in patients with ARVD [39].

Another form of VT occurring in the structurally normal heart is catecholaminergic polymorphic VT. This type of VT should be suspected when syncope triggered by emotion or physical effort occurs in young patients with normal heart and QT interval. First line treatment is BBs. Hypertrophic cardiomyopathy (HCM), a common cause of SCD in young athletes, is a heterogenous group of cardiomyopathy with increased wall thickness. HCM with mid-ventricular obstruction and apical aneurysm is a rare form of HCM which is associated with frequent occurrence of VT. Prophylactic ICD is the main treatment, but RF ablation is required for repetitive VTs [40]. 


\section{Management of ICD repetitive shocks}

Despite ICD can effectively terminate ventricular tachycardia either by antitachycardia pacing or defibrillation shock, it cannot prevent VT recurrences. In patients with ICD, prevention of VT recurrence is required to minimize ICD shocks which can not only be quite uncomfortable for the patient leading to poor quality of life but also cause early battery depletion. Apart from these, recurrent shocks lead to HF progression, frequent hospitalization, and mortality. Use of antiarrhythmic drugs, particularly amiodarone can reduce ICD appropriate shocks by 34\% [41]. In the OPTIC study (the Optimal Pharmacological Therapy in Cardioverter Defibrillator Patients), beta blocker and amiodarone combination were shown to be superior in suppression of VT recurrence compared to BB alone or sotalol [42]. Drug discontinuation rate at 1 year was found to be $18.2 \%$ for amiodarone, $23.5 \%$ for sotalol, and $5.3 \%$ for BB. Mexiletine, a class $1 \mathrm{~b}$ antiarrhythmic drug, was shown to reduce VT recurrence as an adjunct to amiodarone in amiodarone-refractory VT in patients with ICD [43]. Ranolazine, a late Na channel inhibitor, was also shown to reduce VT burden and ICD shocks in patients with drug refractory VT and ICD [44].

Radiofrequency catheter ablation can be lifesaving in patients with ICD and repetitive shocks. In ischemic cardiomyopathy, some trials such as SMASH-VT (Substrate Mapping and Ablation in Sinus Rhythm to Halt Ventricular Tachycardia), VTACH (Ventricular Tachycardia Ablation in Coronary Heart Disease), and VANISH trials have shown the superiority of ablation for reducing ICD shocks [45-47]. The SMASH-VT trial compared ICD implantation plus prophylactic ablation to ICD implantation alone in patients with recent VT. Ablation reduced ICD shocks significantly from 31 to $9 \%$ and reduced VT from 33 to $12 \%$. The VTACH trial assessed the effect of catheter ablation in patients with ischemic cardiomyopathy and mappable VT. Ablation significantly prolonged time to recurrent VT. The VANISH trial compared catheter ablation to escalation of antiarrhythmic therapy on top of first-line antiarrhythmic therapy in patients with VT. Ablation significantly reduced composite outcome of death, appropriate ICD shocks, and VT storm. Repetitive ICD shocks should also warrant referral to an advanced heart failure unit, capable for left ventricular assist device (LVAD) implantation and transplantation [48]. Catheter ablation of VT has risk of complication like all other invasive procedures. Complications related to these procedures are cardiac perforation, systemic embolism including myocardial infarction/stroke, vascular complications, and mortality.

Autonomic modulation procedures may also be applied for VT refractory to ablation. It was shown that videoscopic surgical cardiac sympathetic denervation may reduce the number of ICD shocks in refractory cases [49]. The surgery involves removal of the lower half of the stellate ganglion and T2-T4 stellate ganglia. This technique is especially effective when sympathetic denervation was made bilaterally. Renal denervation was also shown to reduce VT recurrences [50].

Stereotactic body radiation therapy (SBRT) for VT in HF patients has recently emerged as a new way of suppression of VT. SBRT is a technique that delivers high dose of radiation (25 gray) to target tissues with reduced exposure to normal adjacent tissues. SBRT has been used for decades to target various cancers. First, Cuculich et al. showed a 99.9\% reduction in VT burden with cardiac SBRT in a case series of five patients with a high burden of drug-refractory VT, who had been suffering through repeated ICD shocks [51]. And recently, ENCORE VT trial showed that SBRT reduced VT and premature ventricular contraction episodes $94 \%$ at 6 months among 18 patients with treatmentrefractory VT, over half of whom presented with VT storm [52]. 
In selected cases with recurrent VT which cannot be managed with the treatment recommendation given above, implantation of LVAD could temporarily stabilize patient hemodynamically, as well as improve reverse remodeling. LVAD is a battery-operated mechanical pump, which takes the blood from failed LV and pumps it to the aorta to be transmitted to the rest of the body (Figure 4). There are not many heart failure patients with LVAD. However, the management of VT in this patient population requires mention since it is somewhat different than HF patients without LVAD. LVAD may be able to continue maintaining cardiac output in spite of sustained VT, and most of the LVAD patients have ICD in place. When such patients present to the emergency department, first patient hemodynamic status should be assessed. If the blood pressure checked by Doppler ultrasonography is okay, it is reasonable to transfer the patient to a tertiary center where there is LVAD specialist and electrophysiologist. If there is hemodynamic compromise, then the patient should be immediately converted to normal sinus rhythm with electrical shock [53]. If the patient is a candidate neither for transplantation nor LVAD, end-of-life care should be applied for palliation. Shared decision making with the patient and relatives should be done, and discussion regarding measures such as ICD deactivation may be applied in these patients.

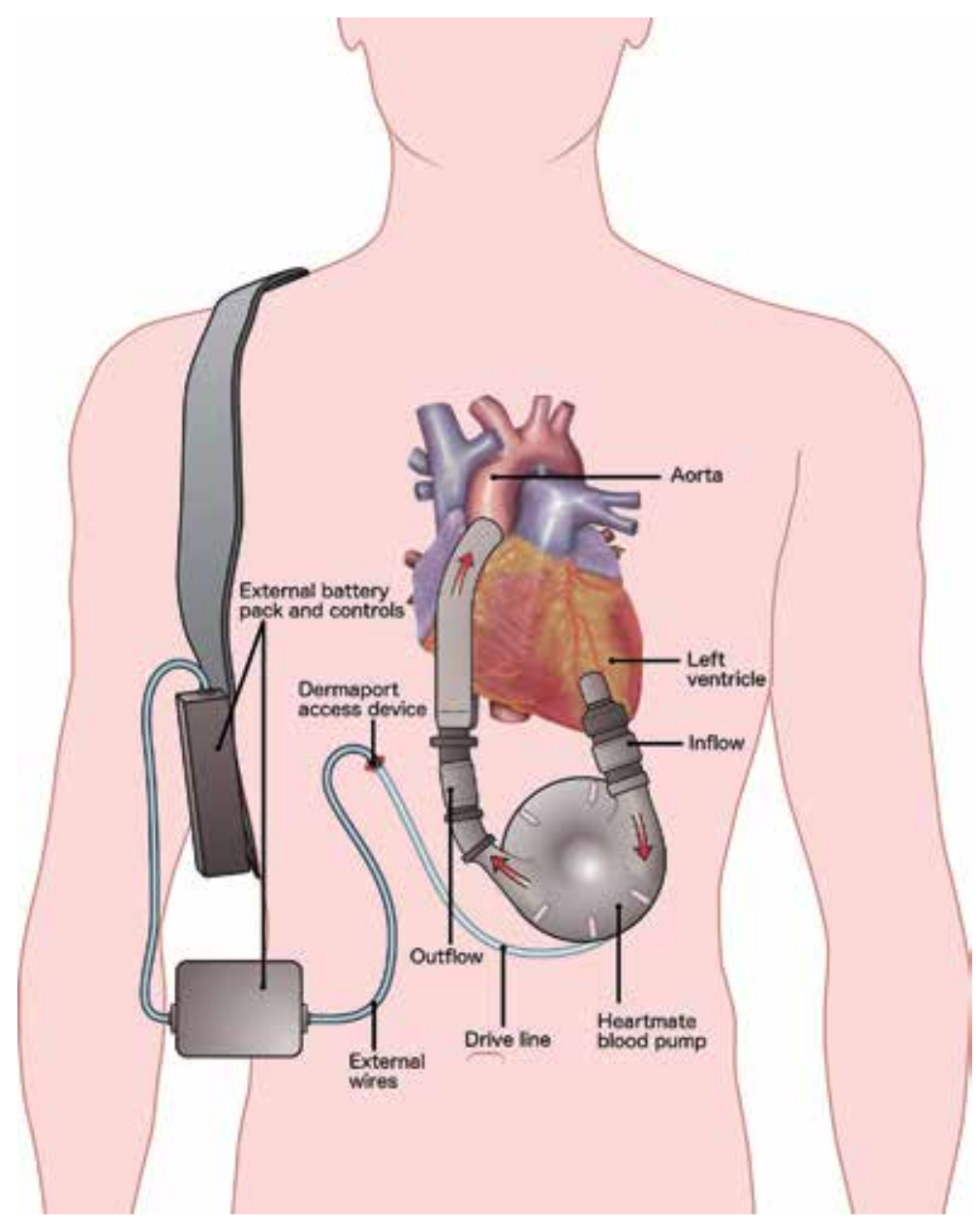

Figure 4 .

A schematic representation of a left ventricle assist device (LVAD) showing battery-operated mechanical pump taking blood from left ventricle and pumping it to aorta. 
VT storm is a medical emergency requiring prompt intervention. Reversible causes of VT, such as hypokalemia, hypomagnesemia, ischemia, and hypoxia should be sought and corrected where applicable. Beta blocker dose should be uptitrated to decrease the sympathetic tone. Another intervention to reduce sympathetic drive is sedation. Radiofrequency catheter ablation has been shown to be effective in controlling VT storm [54].

\section{Conclusion}

Ventricular tachycardia is a frequent event in HF population and is one of the poor prognostic factors related with HF. Management of VT is important because it is associated with SCD which is the responsible cause of death in $50 \%$ of patients with HF. Optimization of guideline-directed treatment is the most important step to prevent occurrence of VT in these patients. ICD has resulted marked improvement in survival of patients with HF and VT. However, repetitive ICD shocks due to recurrent VT poses a great problem and decreases survival. Antiarrhythmic therapy and VT ablation generally offer a complementary treatment in patients with ICD. Patients with VT who have failed standard therapy (antiarrhythmic therapy and catheter ablation) have limited options, with one-year survival below $20 \%$. Autonomic modulation procedures and stereotactic body radiation therapy could be applied in patients with refractory VT. Patients with recurrent VT despite all other measures should be referred to tertiary centers where they are evaluated in respect of indications for LVAD implantation and transplantation.

\section{Author details}

Hakan Altay

Baskent University, Faculty of Medicine, Istanbul Hospital, Turkey

*Address all correspondence to: sakaltay@yahoo.com

IntechOpen

(C) 2019 The Author(s). Licensee IntechOpen. This chapter is distributed under the terms of the Creative Commons Attribution License (http://creativecommons.org/licenses/ by/3.0), which permits unrestricted use, distribution, and reproduction in any medium, provided the original work is properly cited. (cc) BY 


\section{References}

[1] Antiarrhythmics versus Implantable Defibrillators (AVID) Investigators. A comparison of antiarrhythmic-drug therapy with implantable defibrillators in patients resuscitated from nearfatal ventricular arrhythmias. NEJM. 1997;337:1576-1583

[2] Chan MM, Lam CS. How do patients with heart failure with preserved ejection fraction die? European Journal of Heart Failure. 2013;15:604-613

[3] Moss AJ, Greenberg H, Case $\mathrm{RB}$, et al. Long-term clinical course of patients after termination of ven-tricular tachyarrhythmia by an implanted defibrillator. Circulation. 2004;110(25):3760-3765

[4] Kunz R, Burnand B, Schünemann HJ, et al. Das GRADE-system. Der Internist. 2008;49:673-680

[5] Klein L, Hsia H. Sudden cardiac death in heart failure. Cardiology Clinics. 2014;32:135-144

[6] Smer A, Saurav A, Azzouz MS, Salih M, Ayan M, Abuzaid A, et al. Meta-analysis of risk of ventricular arrhythmias after improvement in left ventricular ejection fraction during follow-up in patients with primary prevention implantable cardioverter defibrillators. The American Journal of Cardiology. 2017;120(2):279-286. DOI: 10.1016/j.amjcard.2017.04.020. Epub 2017 Apr 27

[7] Baldinger SH, Stevenson WG, John $\mathrm{RM}$. Ablation of ischemic ventricular tachycardia: Evidence, techniques, results, and future directions. Current Opinion in Cardiology. 2016;31:29-36

[8] Al-Khatib SM, Stevenson WG, Ackerman MJ, Bryant WJ, Callans DJ, Curtis AB, et al. 2017 AHA/ACC/HRS guideline for management of patients with ventricular arrhythmias and the prevention of sudden cardiac death: Executive summary: A Report of the American College of Cardiology/ American Heart Association Task Force on Clinical Practice Guidelines and the Heart Rhythm Society. Heart Rhythm. 2018;15(10):e190-e252. DOI: 10.1016/j. hrthm.2017.10.035. Epub 2017 Oct 30

[9] Tisdale JE, Patel R, Webb CR, Borzak S, Zarowitz BJ. Electrophysiologic and prodysrhythmic effects of intravenous inotropic agents. Progress in Cardiovascular Diseases. 1995;38:167-180

[10] Nakahara S, Chien C, Gelow J, et al. Ventricular dysrhythmias after left ventricular assist device. Circulation: Arrhythmia and Electrophysiology. 2013;6:648-654

[11] Yalagudri S, Zin Thu N, Devidutta S, Saggu D, Thachil A, Chennapragada S, et al. Tailored approach for management of ventricular tachycardia in cardiac sarcoidosis. Journal of Cardiovascular Electrophysiology. 2017;28(8):893-902. DOI: $10.1111 /$ jce.13228

[12] Priori SG, Blomström-Lundqvist C, Mazzanti A, Blom N, Borggrefe M, Camm J, et al. Task Force for the Management of Patients with Ventricular Arrhythmias and the Prevention of Sudden Cardiac Death of the European Society of Cardiology (ESC) 2015 ESC Guidelines for the management of patients with ventricular arrhythmias and the prevention of sudden cardiac death: The Task Force for the Management of Patients with Ventricular Arrhythmias and the Prevention of Sudden Cardiac Death of the European Society of Cardiology (ESC) Endorsed by: Association for European Paediatric and Congenital Cardiology (AEPC). Europace. 2015;17(11):1601-1687. DOI: 10.1093/europace/euv319. Epub 2015 Aug 29 
[13] Al-Gobari M, El Khatib C, Pillon F, Gueyffier F. $\beta$-Blockers for the prevention of sudden cardiac death in heart failure patients: a meta-analysis of randomized controlled trials. BMC Cardiovascular Disorders. 2013;13(52):1-9. DOI: 10.1186/1471-2261-13-52

[14] Pitt B, Remme W, Zannad F, Neaton J, Martinez F, Roniker B, et al. Eplerenone Post-Acute Myocardial Infarction Heart Failure Efficacy and Survival Study Investigators. Epub 2003 Mar 31. Eplerenone, a selective aldosterone blocker, in patients with left ventricular dysfunction after myocardial infarction. The New England Journal of Medicine. 2003;348(14):1309-1321

[15] Moss AJ, Zareba W, Hall WJ, Klein H, Wilber DJ, Cannom DS, et al. Prophylactic implantation of a defibrillator in patients with myocardial infarction and reduced ejection fraction. The New England Journal of Medicine. 2002;346:877-883

[16] Peck KY, Lim YZ, Hopper I, Krum $H$. Medical therapy versus implantable cardioverter-defibrillator in preventing sudden cardiac death in patients with left ventricular systolic dysfunction and heart failure: A meta-analysis of $>35,000$ patients. International Journal of Cardiology. 2014;173(2):197-203. DOI: 10.1016/j.ijcard.2014.02.014. Epub 2014 Feb 22

[17] Zannad F, McMurray JJ, Krum H, van Veldhuisen DJ, Swedberg K, Shi $\mathrm{H}$, et al. Eplerenone in patients with systolic heart failure and mild symptoms. The New England Journal of Medicine. 2011;364:11-21

[18] McMurray JJ, Packer M, Desai AS, et al. Angiotensin-neprilysin inhibition versus enalapril in heart failure. The New England Journal of Medicine. 2014;371:993-1004

[19] Ponikowski P, Voors AA, Anker SD, Bueno H, Cleland JG, Coats AJ, et al.
Authors/task force members; document reviewers.2016 ESC Guidelines for the diagnosis and treatment of acute and chronic heart failure: The task force for the diagnosis and treatment of acute and chronic heart failure of the European Society of Cardiology (ESC). Developed with the special contribution of the Heart Failure Association (HFA) of the ESC. European Journal of Heart Failure. 2016;18(8):891-975. DOI: 10.1002/ejhf.592. Epub 2016 May 20

[20] Cardiac Arrhythmia Suppression Trial (CAST) Investigators. Preliminary report: effect of encainide and flecainide on mortality in a randomized trial of arrhythmia suppression after myocardial infarction. The New England Journal of Medicine. 1989;321:406-412

[21] Bardy GH, Lee KL, Mark DB, Poole JE, Packer DL, Boineau R, et al. Sudden cardiac death in heart failure trial (SCDHeFT) Investigators. Amiodarone or an implantable cardioverter-defibrillator for congestive heart failure. The New England Journal of Medicine. 2005;352:225-237. DOI: 10.1056/ NEJMoa043399

[22] Piccini JP, Berger JS, O’Connor $\mathrm{CM}$. Amiodarone for the prevention of sudden cardiac death: a meta-analysis of randomized controlled trials. European Heart Journal. 2009;30:1245-1253

[23] Bokhari F, Newman D, Greene M, Ko-rley V, Mangat I, Dorian P. Longterm comparison of the implantable cardioverter defibrillator versus amiodarone: Eleven-year follow-up of a subset of patients in the Canadian Implantable Defibrillator Study (CIDS). Circulation. 2004;110:112-116

[24] Waldo AL, Camm AJ, deRuyter H, Friedman PL, MacNeil DJ, Pauls JF, et al. Effect of d-sotalol on mortality in patients with left ventricular dysfunction after recent and remote myocardial infarction. The SWORD 
Investigators. Survival With Oral d-Sotalol. Lancet. 1996;348:7-12

[25] Kober L, Bloch Thomsen PE, Moller M, Torp-Pedersen C, Carlsen J, Sandoe $E$, et al. Effect of dofetilide in patients with recent myocardial infarction and left-ventricular dysfunction: A randomised trial. Lancet. 2000;356:2052-2058

[26] Horowitz LN, Josephson ME, Farshidi A, Spielman SR, Michelson EL, Greenspan AM. Recurrent sustained ventricular tachycardia 3. Role of the electrophysiologic study in selection of antiarrhythmic regimens. Circulation. 1978;58(6):986-997

[27] Connolly SJ, Hallstrom AP, Cappato R, Schron EB, Kuck KH, Zipes DP, et al. Meta-analysis of the implantable cardioverter defibrillator secondary prevention trials. AVID, CASH and CIDS studies. Antiar-rhythmics vs Implantable Defibrillator study. Cardiac Arrest Study Hamburg. Canadian Implantable Defibrillator Study. European Heart Journal. 2000;21: 2071-2078. DOI: 10.1053/euhj.2000.2476

[28] Moss AJ, Zareba W, Hall WJ, Klein H, Wilber DJ, Cannom DS, et al. Multicenter automatic defibrillator implantation trial II investigators. Prophylactic implantation of a defibrillator in patients with myocardial infarction and reduced ejection fraction. The New England Journal of Medicine. 2002;346:877-883. DOI: $10.1056 /$ NEJMoa013474

[29] Kadish A, Dyer A, Daubert JP, Quigg R, Estes NA, Anderson KP, et al. Defibrillators in non-ischemic cardiomy-opathy treatment evaluation (DEFINITE) Investigators. Prophylactic defibrillator implantation in patients with nonischemic dilated. NEJM. 2004;350(21):2151-2158

[30] Sweeney MO, Sherfesee L, DeGroot PJ, Wathen MS, Wilkoff
BL. Differences in effects of electrical therapy type for ventricular arrhythmias on mortality in implantable cardioverter-defibrillator patients. Heart Rhythm. 2010;7:353360. DOI: 10.1016/j. hrthm.2009.11.027

[31] Cleland JG, Daubert JC, Erdmann E, Freemantle N, Gras D, Kappenberger $\mathrm{L}$, et al. Longer-term effects of cardiac resynchronization therapy on mortality in heart failure [the CArdiac REsynchronization-Heart Failure (CARE-HF) trial ex- tension phase]. European Heart Journal. 2006;27:1928-1932

[32] Sapp JL, Parkash R, Wells GA, Yetisir E, Gardner MJ, Healey JS, et al. Cardiac resynchronization therapy reduces ven-tricular arrhythmias in primary but not secondary prophylactic implantable cardioverter defibrillator patients: Insight from the resynchronization in ambulatory heart failure trial. Circulation. Arrhythmia and Electrophysiology. 2017;10. pii: 004875

[33] Muresan L, Cismaru G, Martins RP, Bataglia A, Rosu R, Puiu M, et al. Recommendations for the use of electrophysiological study: Update 2018. Hellenic Journal of Cardiology. 2018S;11099666(18)30352-X:1-19. DOI: 10.1016/j. hjc.2018.09.002

[34] Pagé PL, Cardinal R, Shenasa M, Kaltenbrunner W, Cossette R, Nadeau $R$. Surgical treatment of ventricular tachycardia. Regional cryoablation guided by computerized epicardial and endocardial mapping. Circulation. 1989;80(3 Pt 1):I124-I134

[35] Dinov B, Fiedler L, Schönbauer R, et al. Outcomes in catheter ablation of ventricular tachycardia in dilated nonischemic cardiomyopathy in comparison to ischemic cardiomyopathy: Results from the Prospective HEart Centre of LeiPzig VT (HELP-VT) Study. Circulation. 2014;129:728-736 
[36] Della Bella P, Trevisi N. Catheter ablation of ventricular tachycardia in nonischemic dilated cardiomyopathy: A difficult field where we should focus our efforts. Circulation. Arrhythmia and Electrophysiology 2016;9(10). pii: e004649

[37] Kanagaratnam L, Tomassoni G, Schweikert R, Pavia S, Bash D, Beheiry $\mathrm{S}$, et al. Ventricular tachycardias arising from the aortic sinus of valsalva: An under-recognized variant of left outflow tract ventricular tachycardia. Journal of the American College of Cardiology. 2001;37(5):1408-1414

[38] Fragakis N, Karamitsos TD, Vassilikos V. Successful catheter ablation of an incessant ventricular tachycardia originating from the posterior papillary muscle in a structurally normal right ventricle. Hellenic Journal of Cardiology. 2016;57:286-288

[39] Saguner AM, Medeiros-Domingo A, Schwyzer MA, On CJ, Haegeli LM, Wolber T, et al. Usefulness of inducible ventricular tachycardia to predict long-term adverse outcomes in arrhythmogenic right ventricular cardiomyopathy. The American Journal of Cardiology. 2013;111(2):250-257. DOI: 10.1016/j. amjcard.2012.09.025

[40] Lim KK, Maron BJ, Knight BP. Successful catheter ablation of hemodynamically unstable monomorphic ventricular tachycardia in a patient with hypertrophic cardiomyopathy and apical aneurysm. Journal of Cardiovascular Electrophysiology. 2009;20(4):445-447. DOI: $10.1111 / j .1540-8167.2008 .01366 . x$

[41] Antangeli P, Muser D, Maeda S, et al. Comparitive effectiveness of antiarrhythmic drugs and catheter ablation for the prevention of recurrent ventricular tachycardia in patients with implantable cardioverter-defibrillators: A systematic review and meta-analysis of randomized contolled trials. Heart Rhythm. 2016;13:1552-1559. DOI: 10.1016/j. hrthm.2016.03.004. PMID: 26961297

[42] Connolly SJ, Dorian P, Roberts RS, et al. Comparison of beta-blockers, amiodarone plus beta-blockers, or sotalol for prevention of shocks from implantable cardioverter defibrillators: The optic study: A randomized trial. JAMA. 2006;295:165-171

[43] Gao D, Van Herendael H, Alshengeiti L, et al. Mexiletine as an adjunctive therapy to amiodarone reduces the frequency of ventricular tachyarrhythmia events in patients with an implantable defibrillator. Journal of Cardiovascular Pharmacology. 2013;62:199-204. DOI: $10.1097 /$

FJC.0b013e31829651fe. PMID: 23609328

[44] Bunch TJ, Mahapatra S, Murdock D, Molden J, Weiss JP, May HT, et al. Ranolazine reduces ventricular tachycardia burden and ICD shocks in patients with drug-refractory ICD shocks. Pacing and Clinical Electrophysiology. 2011;34:1600-1606

[45] Reddy VY, Reynolds MR, Neuzil P, et al. Prophylactic catheter ablation for the prevention of defibrillator therapy. The New England Journal of Medicine. 2007;357:2657-2665. DOI: 10.1056/ NEJMoa065457. PMID: 18160685

[46] Kuck KH, Schaumann A, Eckhardt $\mathrm{L}$, et al. Catheter ablation of stable ventricular tachycardia before defibrillator implantation in patients with coronary heart disease (VTACH): A multicentre randomised controlled trial. Lancet. 2010;375:31-40. DOI: 10.1111/jce.12073. PMID: 23350967

[47] Sapp JL, Wells GA, Parkash R, Stevenson WG, Blier L, Sarrazin JF, et al. Ventricular tachycardia ablation versus escalation of antiarrhythmic drugs. The New England Journal of Medicine. 2016;375(2):111-121 
[48] Yancy CW, Januzzi JL Jr, Allen LA, Butler J, Davis LL, Fonarow GC, et al. 2017 ACC expert consensus decision pathway for optimization of heart failure treatment: Answers to 10 pivotal issues about heart failure with reduced ejection fraction: A report of the American College of Cardiology Task Force on Expert Consensus Decision Pathways. Journal of the American College of Cardiology. 2018;71(2): 201-230. DOI: 10.1016/j.jacc.2017.11.025. Epub 2017 Dec 22

[49] Vaseghi M, Barwad P, Malavassi Corales FJ, et al. Cardiac sympathetic denervation for refractory ventricular arrhythmias. Journal of the American College of Cardiology. 2017;69: 3070-3080. DOI: 10.1016/j. jacc.2017.04.035. PMID: 28641796

[50] Bradfield JS, Ajijola OA, Vaseghi M, et al. Mechanisms and management of refractory ventricular arrhythmias in the age of autonomic modulation. Heart Rhythm. 2018;15:1252-1260. DOI: 10.1016/j.hrthm.2018.02.015. PMID: 29454137

[51] Cuculich PS, Schill MR, Kashaniand R, et al. Noninvasive cardiac radiation for ablation of ventricular tachycardia. The New England Journal of Medicine. 2017;377:2325-2336. (Abstract)

[52] Robinson CG, Samson PP, Moore KMS, Hugo GD, Knutson N, Mutic S, et al. Phase I/II trial of electrophysiology-guided noninvasive cardiac radioablation for ventricular tachycardia. Circulation. 2019;139(3):313-321

[53] Fitzgibbon J, Kman NE, Gorgas D. Asymptomatic sustained polymorphic ventricular tachycardia in a patient with a left ventricular assist device: Case report and what the emergency physician should know. The Journal of Emergency Medicine. 2016;50(3):e135-e141
[54] Carbucicchio C, Santamaria M, Trevisi N, Maccabelli G, Giraldi F, Fassini G, et al. Catheter ablation for the treatment of electrical storm in patients with implantable cardioverterdefibrillators: Short and long-term outcomes in a prospective single-center study. Circulation. 2008;117:462-469 



\title{
Genetic Polymorphisms that Playing Role in Development of Hypertrophic Cardiomyopathy
}

\author{
Nevra Alkanli and Arzu Ay
}

\begin{abstract}
Hypertrophic cardiomyopathy (HCM) is a complex heart disease with various physiopathological, morphological, functional, and clinical features. In this disease, HCM is known to be an autosomal genetic disease in more than half of the cases. Mutations in sarcomeric genes are thought to play an important role in the pathogenesis of the disease. Modifying genes and environmental factors also together affect the phenotypic expression and severity of HCM. The phenotypic expression of HCM is determined by causal sarcomeric gene mutations and the regulatory genetic basis of genes. HCM, a multi-factorial disease, involves the effects of many environmental gene modifiers and the sarcomeric/cytoskeletal genes. The single nucleotide polymorphisms occurring in the human genome differ in terms of susceptibility to disease in various populations. Therefore, the determination of genetic polymorphisms involved in the development of HCM disease is very important for the diagnosis of the disease.
\end{abstract}

Keywords: hypertrophic cardiomyopathy, gene polymorphisms, LVH, PCR

\section{Introduction}

HCM is a complex cardiac disease with major clinical heterogeneity and diagnostic and prognostic effects specific to each mutation. At the same time, this disease has different physiopathological, morphological, functional, and clinical features. HCM, with left ventricular hypertrophy $(\mathrm{LVH})$, is a primary cardiac disorder and occurs when there is no cardiac or systemic disease. Throughout life, it is known that it has a clinical course ranging from symptomatic patients to heart failure symptoms and sudden deaths. It is an autosomal dominant genetic disease in more than half of cases, but it still does not have a fully defined etiology [1]. Modifying genes and environmental factors play an important role in the pathogenesis of HCM. Phenotypic expression and the formation of cardiovascular events are affected by means of these factors [2]. The cardiac $\beta$-myosin heavy chain (MYHC) gene, cardiac troponin T (cTnT) gene, $\alpha$-tropomyosin gene, myosinbinding protein $\mathrm{C}$ (MYBP-C) gene, cardiac troponin I gene, and regulatory and essential myosin light chain genes are found among genes encoding the proteins of sarcomere [1]. These genes encoding sarcomeric proteins are localized on different chromosomes. It is known that the first gene identified from these genes is the $\beta$ MYHC gene encoding the major contractile protein. In HCM patients, due to 
defects in sarcomeric proteins, mutations such as MYBP-C, $\alpha$-tropomyosin, cTnT, ventricular myosin essential and regulatory light chains, cardiac troponin I, and cardiac $\alpha$-actin and titin have been described. This disease, known to be caused by the defects in sarcomeric proteins, is called sarcomere disease [3]. In addition to mutations in sarcomeric and non-sarcomeric genes, many other gene mutations also lead to metabolic disorders with similar phenotypes in HCM [4]. So far, more than 1400 mutations have been identified in many genes, and the most important genes of these mutations have been identified to encode the protein components of cardiac sarcomere that perform contractile, structural, and regulatory functions [5]. The purpose of this chapter, in addition to giving general information about $\mathrm{HCM}$, is to summarize the studies that investigated the relationship between gene polymorphisms that play a role in the development of HCM and the risk of developing HCM.

\section{Hypertrophic cardiomyopathy}

HCM, a cardiac disease, is characterized by marked hypertrophy and genetic variability. It is known as a disease characterized by LVH which may cause primary or systemic hypertrophy when there is no other disease [6]. LVH, known as a physiological adaptation to increased workload of the heart, usually develops in clinical conditions such as hypertension, valvular disease, and myocardial infarction. In some patients, cardiac hypertrophy develops when there are no clinical conditions causing cardiac overload. This condition is considered to be the basic form of $\mathrm{LVH}$, and it is thought that this form, which is frequently familial, is caused by mutations in sarcomeric genes. This form of the most common hereditary heart disease is defined as HCM [7]. HCM is one of the leading causes of sudden deaths in young people and athletes. One person in 500 people worldwide is affected by this disease [6]. HCM is a common heterogeneous disease with high morbidity and mortality in the elderly, and it is characterized by enlarged heart, abnormally thickened left ventricular walls, and reduced chamber capacity [8]. Histologically, in this disease, characterized by left ventricular thickness resulting from cardiomyocyte hypertrophy, cardiomyocytes lose their cleavage ability in the first week after birth. Thus, cardiomyocyte hypertrophy is effective instead of cardiomyocyte proliferation in postnatal growth of the heart. Postpartum cardiac growth is a physiological response of myocardium to stress signals as well as its role in cardiomyocyte hypertrophy. It is known that the response of cardiomyocytes to stress signals is characterized by reactivation of fetal gene program [4]. This disease is thought to be caused by contractile proteins encoding genes that cause contractile dysfunction and then hypertrophy. Familial HCM, defined as an autosomal dominant disorder, is usually disseminated by incomplete penetrance due to heterozygous pathogenic gene mutations [8]. HCM is a genetically transmitted, cardiovascular disease with heterogeneous clinical features. Sudden cardiac death in HCM occurs as a frequent complication of $2-3 \%$ per year. HCM can be seen form of autosomal dominant feature as a familial disorder; on the other, it can also occur as a sporadic disease that may develop due to novo mutations. These familial and sporadic forms represent different parts of the same spectrum. According to phenotypic models, HCM phenotypes, asymmetric septal hypertrophy (ASH), apical hypertrophy $(\mathrm{AH})$, diffuse hypertrophy $(\mathrm{DH})$, and left ventricular free wall hypertrophy $(\mathrm{FH})$ are classified as. The etiology of the disease is multifactorial, and the majority of cases occur due to secondary mutations in sarcomere myofilament genes. The sarcomere myofilament genes are genes that contribute to heterogeneity in the phenotype of the disease. HCM has a 
wide familial variability ranging from severe symptomatic individuals to asymptomatic individuals [4]. Cardiac phenotype and variability in clinical course not only depend on pathogenic genes but also depend on environmental factors [3]. Important information can be obtained in terms of prognosis and treatment of the disease through the identification of these environmental and genetic factors [4].

\section{Genetic polymorphisms}

\subsection{Sarcomeric gene polymorphisms}

\subsection{1 $\beta M Y H C$ versus MYBP-C gene polymorphisms}

Mutations in cardiac $\beta$-myosin encoded by the MYH7 gene and myosin-binding protein $\mathrm{C}$ sarcomere proteins encoded by MYBC3 gene have been associated with the development of HCM disease. Mutations in these genes are responsible for $50-70 \%$ of HCM's genetic cases. $\beta$-myosin is a large protein containing 1935 amino acids and is localized on chromosome 14 (14q11) in human. During muscle contraction 2q13 interacts with the thin filament, and this gene consists of 40 exons. The MYBP-C gene is also localized on chromosome 11 (11p.11.2), and 14 mutations have been identified in this gene so far. It has been reported that four of these mutations to be caused by nucleotide changes and eight of which by truncated mutations. The most important feature of these mutations is moderate hypertrophy and low penetration until a certain period of life. About 40 mutations have been identified in $\beta$-MYHC that may cause disease, and it is known that most of these mutations occur as a result of the translocation of DNA nucleotides. Displacement in nucleotides also causes amino acid changes in the protein sequence. This change is particularly observed in the familial form of HCM. Among these 40 mutations, there are mutations with high, medium, and low sudden death risks. Arg403Gln, Arg453Cys, and Arg719Gln mutations are known to be malignant. Arg403Gln-related phenotypes were observed in many families, and it is determined that these phenotypes were associated with high penetrance, high incidence of sudden death, and severe hypertrophy. Glu930Lys and Arg249GIn polymorphisms were associated with the middle risk of sudden death. However, Leu908Val, Gly256Glu, Val606Met, and Fhe513Cys polymorphisms have been reported to be associated with benign prognostic and normal survival. Myosin, a hexameric protein, consists of two heavy and two light chains. Light chains contain two light chains as the regulatory light chain (RLC) and the basic light chain (ELC). The myosin heavy chain is also divided into three parts as the lower part 1 (S1), the lower part 2 (S2), and the light meromyosin (LMM). Regulatory and essential myosin light chains were first found in 1996. The regulatory MYL2 gene is localized on chromosome 12 (12q23-q24.3). The essential gene is localized on chromosome 3 (3p) [1]. A large number of mutations have been identified in most S1 and S2 regions, which are associated with HCM in the MYH7 gene. The frequency of these mutations is variable and is known to be associated with marked hypertrophy. MYH7 and MYBPC3 genes are responsible for about $70 \%$ of genotyped HCM cases. Mutations in the MYH7 gene are missense mutations and localized at the head of globular myosin. The MYH7 gene is also known to be associated with dilated cardiomyopathy, and a large number of mutations have been identified in the rod region of the gene. There are many studies that demonstrate a relationship between mutations in the MYH7 gene and the family history of HCM. Studies to investigate MYH7 mutations have generally been limited by the analysis of regions encoding the head and neck domains of $\beta$ MYHC. However, it is 
determined that mutations in the tail region of the protein may be related to the risk of developing HCM. Mutations in the MYH7 gene are known as a cause of HCM, and sudden death was significantly higher in the family history and in patients with severe left ventricular hypertrophy. In a previous study, four new mutations are identified. In some of these mutations, the relationship between genotype and phenotype is constant. There are significant differences between phenotypes in other mutations [9]. In a study conducted with the Venezuelan population, no missense mutation identified in the MYH7 gene was found. In the same study, the frequency of mutation of MYH7 gene in adult HCM patients was found to be low. In another study performed by Ronkaratti et al., in the Italian population, it is found that the frequency of mutations determined in the MYH7 gene was found to be very low. Many factors, such as modifying genes, epigenetic factors, microRNAs, posttranslational protein modifications, and environmental factors, may affect the clinical course of HCM disease $[1,10]$. Genetic studies are required to understand the clinical and prognostic heterogeneity of HCM. The obtained information of clinical and morphological characteristics of different mutation carriers is important in terms of clinical decision-making in their genetic studies [9].

\subsubsection{Cardiac troponin T gene polymorphisms}

The cardiac troponin $\mathrm{T}$ gene is localized on chromosome 1 (1q3), and so far eight mutations have been identified in this gene. The most important feature of these mutations is that they cause hypertrophy and high incidence of sudden death in younger patients under 30 years of age [1].

\subsubsection{Alpha tropomyosin gene polymorphisms}

Alpha tropomyosin gene is localized on chromosome 15 (15q2), and two mutations of this gene have been observed to date. It is observed in a low proportion of $\mathrm{HCM}$ cases and is known to be associated with normal survival [1].

\subsection{TLR4 gene polymorphisms}

Studies have shown that the immune system and multiple proinflammatory factors play an important role in the pathogenesis of HCM. Toll-like receptor 4 (TLR4), a member of the pattern recognition receptors, plays an important role as mediation in inflammatory response. TLR4 consists of three exons involved in immunoregulation and is localized in region 9q32-q33. It acts by suppressing T lymphocyte proliferation and regulating macrophage function. The lack of TLR4, which is known to play an important role in the development of cardiovascular diseases, has been reported to be associated with doxorubicin-induced cardiomyopathy in mice. A significant relationship between abnormal expression or genetic polymorphisms and cardiovascular remodeling, which is considered to be an important risk factor for metabolic syndrome, has been found. TLR4, which can trigger protein kinase signaling and innate immune response active with mitogen, leads to activation of proinflammatory cytokines and Chemokines. Common polymorphisms in the TLR4 gene are the rs4986791 and rs4986790 polymorphisms. In addition to these polymorphisms, new polymorphisms have been identified in the promoter region and in the $3^{\prime}$ untranslated region ( $3^{\prime}$-UTR) of TLR4. Even if the polymorphisms occurring in the promoter region do not alter the gene's coding sequence, the initiation of gene transcription is affected when these gene polymorphisms lead to pathogenicity. Cohort studies aimed at determining the relationship between cardiovascular diseases and TLR4 gene polymorphisms were performed, and inconsistent results were 
obtained in these studies. In a study by Lindstrom et al., TLR4 gene polymorphisms were associated with decreased risk of prostate cancer. In another study by Castano et al., TLR4 was found to be associated with increased gastric cancer risk in the 3'UTR region. In a study by Kiechl et al., it was concluded that TLR4 gene polymorphisms were associated with heart diseases such as atherogenesis. No large number of studies have been carried out to determine whether TLR4 gene polymorphisms are genetic risk factors for HCM. In a study conducted with the Han-China population, TLR4 gene polymorphisms were found to be genetic risk factors in the development of HCM. In this study, it was determined that rs11536865 and rs10983755 gene polymorphisms in the promoter region of the TLR4 gene are important risk factors for HCM development. In this study, the potential relationships of TLR4 gene polymorphisms with the sensitivity and prognosis of HCM have been revealed. It is determined that it is associated with decreased plasma TLR4 levels of the GG genotypes of $-728 \mathrm{G}>\mathrm{C}$ polymorphism and GG genotypes of $-2081 \mathrm{G}>$ A polymorphism in HCM patients. However, the $\mathrm{C}$ allele of the $-728 \mathrm{G}>\mathrm{C}$ polymorphism and the A-allele of $-2081 \mathrm{G}>\mathrm{A}$ polymorphism were found to be related to the highest plasma TLR4 levels. Inflammation and innate immunity are also contributed to the development of cardiomyopathy. Accordingly, it is believed that TLR4 gene polymorphisms affect the progression of natural immunity or inflammation, thus altering the expression level of TLR4, which is involved in the development of HCM. When studies with larger populations are performed, different results are likely to occur [8]. Primer sequences for $-728 \mathrm{G}>\mathrm{C}$ and $-2081 \mathrm{G}>\mathrm{A}$ gene polymorphisms are presented in Table 1.

\subsection{HOPX gene polymorphisms}

Homeodomain only protein $\mathrm{x}(\mathrm{HOPX})$ is a homeodomain protein that regulates the serum response factor (SRF)-dependent gene expression. In addition, HOPX is thought to play a role as tumor suppressor gene in some tissues, and expression is silenced in human carcinomas such as choriocarcinoma, lung cancer, head and neck squamous carcinoma, and esophageal cancer during cardiac hypertrophy, SRF activity, which controls the transcription of genes, including cellular immediateearly genes, and cell skeletal and contractile proteins, is controlled by cofactors such as myocardium and compressors such as HOPX. The expression of the HOPX gene encoding a homeodomain protein is under the control of the two promoter regions. One of these promoters is regulated by the cardiac-specific transcription factor Nkx2-5. The HOPX gene plays a role as SRF antagonist, and it is effective in prenatal cardiomyocyte proliferation and postnatal cardiomyocyte hypertrophy. This antagonistic effect performed through by the take of histone deacetylase. In addition, HOPX is thought to play a role as tumor suppressor gene in some tissues, and expression is silenced in human carcinomas such as choriocarcinoma, lung cancer, head and neck squamous carcinoma, and esophageal cancer. HOPX has a role coactivator on SRF activity. Through this, it plays an active role in cardiac hypertrophy. HOPX gene expression is known to be downregulated in kalp insufficiency, but

\begin{tabular}{lll}
\hline SNPs & Forward primer $\left(5^{\prime}-\mathbf{3}^{\prime}\right)$ & Reverse primer $\left(\mathbf{5}^{\prime}-\mathbf{3}^{\prime}\right)$ \\
\hline$-728 \mathrm{G}>\mathrm{C}$ & 5'-TGATAGACCCCACAACTCCT-3' $^{\prime}$ & 5'-TGATTTCCCCC-CATAGGATG-3' $^{\prime}$ \\
\hline$-2081 \mathrm{G}>$ A & 5'-TACCACCACTGTTCGCTCAG-3' & 5'-GGTTATGAGGGACATTGGAT-3' $^{\prime}$ \\
\hline PCR, polymerase chain reaction; SNP, single nucleotide polymorphism. & \\
\hline
\end{tabular}

Table 1.

Primer sequences used in PCR for TLR4 gene polymorphisms. 
the association between gene polymorphisms in the HOPX gene and heart disease such as heart failure or HCM has not been well established. HOPX protein is not a component of sarcomere and plays a role as a modifying gene. In a study investigating the relationship between HOPX gene polymorphism and SRF-dependent gene expression, it was determined that the expression decreased in heart muscles of mutant mice. Sequence variations in the HOPX gene in particular in the regulatory region have been shown to be associated with HCM. The relationship between HOPX and syncope in HCM is classified in two ways as dependent on SRF and independent on SRF. The HOPX gene plays a modifying role in HCM pathogenesis through SRF-dependent genes, and it is thought that the modifying effect may be more pronounced in patients with mutations in target genes. In a previous study performed in HCM patients, no mutation was detected in the coding sequence of the HOPX gene, but two noncoding polymorphisms associated with syncope were detected. In these polymorphisms, it is determined that homozygous states are protective against syncope and heterozygote cases are a genetic risk factor for syncope. The epigenetic status and genetic variations of the HOPX gene are important as modifying factors in HCM [4]. Primer sequences for HOPXe1, HOPXe2, and HOPXe3 gene polymorphisms are presented in Table 2 .

\subsection{PRKHC gene polymorphisms}

The PRKCH gene is a susceptibility gene that plays an important role in atherosclerotic diseases such as cerebral infarction and is associated with the development and progression of atherosclerosis in humans. This gene encodes protein kinase $C$ (PKC), and $\mathrm{PKC}$ is activated by diacylglycerol which calcium and secondary messenger. Protein kinase C (PKC) functions as an important signal transduction pathway in the development of cardiac hypertrophy, and studies performed with cell culture and animal models explain this function. It is serine-threonine kinase which is effective in regulating various important cellular functions including proliferation, differentiation, and apoptosis. Members of the PKC family which phosphorylate a wide variety of protein targets are associated with several signalization pathways. There are studies showing that PKC activation is important in the pathology of cardiovascular diseases. The PRKCH gene is located in the ATP-binding region of PKC $\eta$ in exon 9. PKC $\eta$, expressed in the skin and heart tissues, is effective by the way of contributing to cellular processes such as proliferation, differentiation, secretion, and apoptosis. $\mathrm{PKC} \eta$ also plays an important role in immune functions such as regulation of TLR2 responses in macrophages, T-cell proliferation, and homeostasis. The 1425G/A (Val374Ie) polymorphism in the PRKCH gene localized on 14q22-q23 in human increases the kinase activity. In a study conducted by Centurione et al., PKC $\eta$ has been reported to regulate hypertrophic and apoptotic events, NF-Kb signaling system, and intrinsic mitochondrial apoptotic pathway in rat neonatal heart. In a study conducted with a Chinese population, the PRKCH 1425G/A gene polymorphism was found to be a genetic risk factor in the development of hypertrophic obstructive cardiomyopathy (HOCM). In studies conducted with Chinese and

\begin{tabular}{lll}
\hline SNPs & Forward primer $\left(5^{\prime}-3^{\prime}\right)$ & Reverse primer $\left(5^{\prime}-3^{\prime}\right)$ \\
\hline HOPXe1 & 5'-AACGTGCTATCAGCAGCCTG-3' $^{\prime}$ & 5'-GACGAACAGGACCGCCCAGC-3' $^{\prime}$ \\
\hline HOPXe2 & 5'-CGACCGCCTTCCTTCGCTGC-3' $^{\prime}$ 5'-CCTTCATGGAGTGAAGCTGTC-3' \\
\hline HOPXe3 & 5'CTTGTGCCACAGAGGCTACC-3' $^{\prime}$-CT'-CCTTCATGGAGTGAAGCTGTC-3' \\
\hline PCR, polymerase chain reaction; SNP, single nucleotide polymorphism. &
\end{tabular}

Table 2.

Primer sequences used in PCR for HOPX gene polymorphisms. 


\begin{tabular}{cll}
\hline SNPs & Common primer $\left(5^{\prime}-\mathbf{3}^{\prime}\right)$ & $\begin{array}{l}\text { Allele-specific primer (A) } \\
\text { Allele-specific primer (G) }\end{array}$ \\
\hline PRKCH & 5'-GCAGAATCACGTCCTTC & 5'-CATAGGTGATGCTTGCAAGAA-3' \\
\cline { 2 - 2 } 1425G/A & TTCAG-3' & 5'-CATAGGTGATGC TTGCAAGAG-3' \\
\hline
\end{tabular}

$P C R$, polymerase chain reaction; SNP, single nucleotide polymorphism.

Table 3.

Primer sequences used in PCR for PRKCH gene polymorphism.

Japanese populations, PRKCH 1425G/A gene polymorphism was found to be associated with increased ischemic stroke and the risk of cerebral hemorrhage. More studies should be performed related to molecular mechanisms to determine the relationship between the risk of developing PRKCH and HOCM [5]. Primer sequences for PRKCH 1425G/A gene polymorphisms are presented in Table 3.

\subsection{SCN10A gene polymorphisms}

The SCN10A gene encodes NaV1.8, a neuronal sodium channel isoform. NaV1.8 is an alpha subunit of sodium channels with voltage door. NaV1.8 localized in the peripheral nervous system is associated with chronic and neuropathic pain. With rapid and sustained stimulation, long-term action potential is observed and excitability is maintained. SCN10A identified in the human heart was found to be associated with changes in cardiac and atrioventricular conduction. Significant relationships were found between the PR interval, QRS duration, and SCN10A gene polymorphisms in recent genome-wide association studies. Starting from this, it is concluded that NaV1.8 plays an important role in cardiac electrophysiology. In a study by Chambers et al., rs6795970 gene polymorphism has been shown to result in the amino acid exchange A1073V in the IDII/III intracellular cycle of NaV1.8. In another study, it was determined that the A-allele of the rs6795970 gene polymorphism occurring in the SCN10A gene may be related to the cardiac conduction abnormalities observed in HCM patients. In addition, significant correlations were found between the A-allele of the rs6795970 gene polymorphism and the increase in the risk of first-degree heart block, bundle brunch block, and bifascicular heart block [2].

\subsection{HSP 70 gene polymorphisms}

Heat shock protein 70 (HSP 70) is localized on 6p21.3 and is located in the class III region of the major histocompatibility complex (MHC). This gene is expressed in response to heat shock and stress stimulators such as oxidative free radicals and toxic metal ions. Some of the HSPs play an important role in controlling protein folding, translocation, or degradation and are structurally expressed in non-stressed cells. There are three gene modifiers such as HSP 70-1, HSP 70-2, and HSP 70-Hom. HSP 70-1 and HSP 70-2 are those that encode an identical protein of the heat-inducible HSP 70. HSP 70-Hom is expressed at structurally low levels; it encodes a protein non-inducible with heat. There are studies showing that the overexpression of heat shock proteins has a cardioprotective role and that genetic variants of HSP 70 may reduce the ability of cells to protect against ischemia. The genetic polymorphisms in the HSP 70 gene have been found to play an important role in various diseases such as Parkinson's disease, schizophrenia, breast carcinoma, ischemic stroke, and coronary artery disease. The relationship between HSP 70 specific genotypes and hypertrophic cardiomyopathy has not been reported so far. In a previous study, the modifying role of HSP 70 has been described. In the study, it was found that HSP plays a regulatory role in HCM-related inflammatory 
responses and hemodynamic compensatory mechanisms. HSP genes are genes that encode a family of structurally produced proteins in the fulfillment of basic functions, which increase expression in response to various metabolic stimuli. One of the most important tasks of these genes is to facilitate the synthesis and folding of proteins within the cells. In addition HSP genes play an important role in protein binding, secretion, protein degradation, and in the regulation of protein kinases via transcription factors. Polymorphisms in the expression of HSP genes are controlled by a number of transcription factors, and these factors are called heat shock factors (HSF). As a result of the increase and accumulation of HSPs, the protection of the stressed cell is increased; thus the cell survival is maintained. Overexpression of HSP 70 elicits its cardioprotective property. As a result of the polymorphisms occurring in the HSP 70 gene, the synthesis of HSP 70 protein can be changed [11].

\subsubsection{HSP 70-1 (+190G/C) polymorphism}

The HSP 70-1 (+190G/C) polymorphism is a silent polymorphism of the initial domain translated in the $5^{\prime}$-UTR region of the gene. It has been reported to be a significant relationship between this polymorphism and various diseases such as Parkinson's disease, high-altitude illness, and diabetes mellitus. HSP 70 is known as a significant stress protein whose production is increased under stress [11].

\subsubsection{HSP 70-2 (+1267A/G) polymorphism}

The HSP 70-2 (1267A/G) polymorphism is a polymorphism located in the coding region of the gene. HSP 70-2 changes the expression of mRNA, and the relationship between this expression and the $+1267 \mathrm{~A} / \mathrm{G}$ polymorphism is shown in several studies. In a study, G allele of HSP 70-2 (1267A/G) polymorphism was found to be an important risk factor in the development of HCM. In a study by Pociot et al., it was determined that the differences between individuals in HSP 70 expression may be related to different regulatory mechanisms than transcriptional regulation. In addition, the HSP 70 polymorphic region affects expression and enzyme activity of the synonymous gene polymorphism. As a result of changing the timing of co-translational folding, the secondary structure of mRNA, stability, substrate, or inhibitor binding sites of the brain vary. In a study, there was no change in the secondary structures of the A and G alleles of HSP 70-2 mRNA [11].

\subsubsection{HSP 70-Hom (+2437C/T) polymorphism}

The HSP 70-Hom $(+2437 \mathrm{C} / \mathrm{T})$ polymorphism is characterized by the Met493Thr missense translocation, which affects the substrate specificity and chaperone activity of HSP 70-Hom. In a study conducted with a Mexican population, a significant relationship was found between the $+2437 \mathrm{~T}$ allele and spondyloarthropathies of HSP 70-Hom $(+2437 \mathrm{C} / \mathrm{T})$ gene polymorphism. It is known that nucleotide changes in the coding region may influence the peptide binding kinetics and the affinity of ATPase activity with HSP 70 proteins. Furthermore, as a result of the nucleotide changes that occur in the side regions, the inducibility, expression grade, and stability of mRNA can be affected. Overexpression of HSP 70 is a preservative against the damaging effects of ischemia. In consequence of excessive expression, the release of the creatine kinase of the heart, recovery of high-energy phosphate depots, and correction of metabolic acidosis are performed. Protective effects of HSP include protein folding, abnormal protein degradation, inhibition of apoptosis, preservation of the cell skeleton, and improved NO synthesis. Apoptosis, a programmed cell death involving the release of cytochrome $\mathrm{c}$, is an important consequence of 
Genetic Polymorphisms that Playing Role in Development of Hypertrophic Cardiomyopathy DOI: http://dx.doi.org/10.5772/intechopen.83473

\begin{tabular}{lll}
\hline SNPs & Forward primer $\left(5^{\prime}-3^{\prime}\right)$ & Reverse primer $\left(5^{\prime}-3^{\prime}\right)$ \\
\hline $\begin{array}{l}\text { HSP 70-1 } \\
\text { +190G/C }\end{array}$ & $5^{\prime}$-CGCCATGGAGACCAACACCC-3' & $5^{\prime}$-GCGGTTCCCTGCTCTCTGTC-3' \\
\hline $\begin{array}{l}\text { HSP 70-2 } \\
\text {-1267A/G }\end{array}$ & $5^{\prime}$-CATCGACTTCTACACGTCCA-3' & 5'-CAAAGTCCTTGAGTCCCAAC-3' \\
\hline HSP 70-hom & 5'-GTCCCTGGGGCTGGAGACG-3' & 5'-GATGATAGGGTTACACATCTGCT-3' \\
-2437T/C & & \\
\hline PCR, polymerase chain reaction; SNP, single nucleotide polymorphism. & \\
\hline
\end{tabular}

Table 4.

Primer sequences used in PCR for HSP 70 gene polymorphisms.

hypertrophy decompensation. HSP 70 expression and activation of procaspase 9 , leading to cardiac hypertrophy, can inhibit caspase-mediated apoptosis activity. Hence, the expression of HSP proteins is affected by HSP 70 genes and polymorphisms in these genes. Thus, the ability to inhibit apoptosis resulting in HCM due to cardiac hypertrophy may be affected. In a previous study, the $C$ allele of the HSP 70-1 gene polymorphism and the G allele of the HSP 70-2 gene polymorphism were found to be associated with increased risk of HCM [11]. Primer sequences for HSP-70-1 +190G/C, HSP-70-2 -1267A/G, and HSP-70-hom -2437T/C gene polymorphisms are presented in Table 4.

\subsection{RAAS gene polymorphisms}

The renin-angiotensin-aldosterone system (RAAS) can cause ventricular hypertrophy through circulating angiotensin. RAAS plays an important role in cell proliferation, regulation, and the partial expression of heart hypertrophy, thereby developing $\mathrm{LVH}$ [3]. It is also known to play a regulatory role in cardiac function, blood pressure, and electrolyte homeostasis in the body. Angiotensinogen (AGT), renin, angiotensin-converting enzyme (ACE), and angiotensin II receptors of RAAS are found in the heart, and these components function more independently than circulating RAAS [7]. Angiotensin I is converted to angiotensin II through ACE, and angiotensin II is linked to type 1 receptor angiotensin II (AGTR1). Angiotensin II has an important role in supporting cell growth and hypertrophy. In addition, angiotensin II is converted to aldosterone by aldosterone synthase (CYP11B2), and aldosterone supports cardiac fibrosis. Aldosterone plays an important mediator role in HCM, among sarcomeric mutations and cardiac phenotypes [12]. RAAS activation or receptor function may increase as a result of genetic polymorphisms in genes encoding RAAS. In some studies, a significant relationship was found between RAAS gene polymorphisms and increased hypertrophic response against HCM. In some studies, RAAS gene polymorphisms have been found to be genetic risk factors in the development of $\mathrm{LVH}$, but there are studies that have not confirmed this. Childhood HCM is an early onset HCM and it shows a rapid progress. Furthermore, a growing heart shows more dependence on RAAS than the adult heart. Therefore, it is thought that the growing heart may be more sensitive to RAAS gene polymorphisms. Although studies have shown that there is a relationship between RAAS and HCM, in some studies with different populations, the role of RAAS in the change of HCM phenotype is not well-known [7]. It is thought that modifier genes that regulate RAAS may alter the responses to drug therapies and hence may be effective in the prognosis of HCM patients. RAAS, which is known to be associated with hypertrophy in familial HCM, has been shown to be more effective in sporadic HCM. Early diagnosis of genetic risk factors such as RAAS gene polymorphisms in terms of risk classification and development 
of new strategies for interventions to individual according to this classification are very important [13].

\subsubsection{ACE gene polymorphisms}

ACE increases the synthesis of angiotensin II by inducing cell proliferation, migration, and hypertrophy. Angiotensin II develops the proinflammatory cytokines and matrix metalloproteinases. Therefore, overexpression of angiotensin II is thought to play an important role in cardiomyopathy. ACE, which converts angiotensin I to angiotensin II, functions as a growth factor for cardiac myocytes. It has been reported to induce the cardiac hypertrophy independent of hemodynamic and neurohumoral effects. The ACE gene is localized on chromosome 17 (17q23.3) in the human genome. The gene, which is 21 kilobase in length, consists of 26 exons. The ACE insertion/deletion (I/D) gene polymorphism corresponds to a repetitive sequence of 287 base pairs (Alu) in intron 16 . DD genotype of ACE (I/D) gene polymorphism was found to be associated with increased ACE and angiotensin II levels. This causes increased hypertrophy and fibrosis. Phenotypic expression of HCM is also affected as a result of increase of angiotensin II levels. Previous studies have shown a significant relationship between ACE (I/D) gene polymorphisms and plasma angiotensin II levels. ACE (I/D) gene polymorphism has been shown to modulate the phenotype in HCM patients. In studies conducted with different populations, contradictory results were found in terms of the relationship between ACE (I/D) gene polymorphisms and the risk of developing HCM. In a study performed in Japanese population by Yamada et al., no significant relationship was found between ACE (I/D) gene polymorphism and HCM. In a study by Perkins et al., it was determined that the DD genotype of the ACE (I/D) gene polymorphism was important in the phenotypic expression of HCM and the ACE tissue levels were higher in patients with DD genotype. In another study carried out by Schunkert et al., a significant association was found between D allele of the ACE (I/D) gene polymorphism and increased LVH in HCM patients. In a study conducted by Rai et al., in the Indian population, ACE (I/D) gene polymorphism was found to be a genetic risk factor for HCM and dilated cardiomyopathy. In a metaanalysis study, D allele of ACE (I/D) gene polymorphism has been reported to be associated with increased risk of HCM. In patients with HCM that carry the DD genotype of the ACE (I/D) gene polymorphism, higher serum ACE levels, increased risk of sudden death, and higher severity of hypertrophy are observed than other genotypes. Angiotensin II, which shows trophic effects on the heart, also plays an important role in the development of myocardial hypertrophy. The AGTR1 antagonist has an important role in reducing myocardial hypertrophy, so it may be an important treatment option to prevent the sudden cardiac death in patients with HCM. It is thought that obtaining different results in the studies is due to differences in research design, environmental backgrounds, genetic structure, or sample selection criteria in studies. Further genome-wide relationship studies are needed to determine the relationship between the ACE gene and $\operatorname{HCM}[3,7]$.

\subsubsection{AGTR1 and AGTR2 gene polymorphisms}

$\mathrm{LVH}$ is known to be variable in patients with HCM. Angiotensin II plays an important role in the change of LVH. AGTR1 A1166C and angiotensin II type 2 receptor (AGTR2). As a result of A3123C gene polymorphisms, phenotypic expression of hypertrophy in HCM is affected. The AGTR1 gene is localized on 
chromosome 3q21. AGTR1 A1166C gene polymorphism is characterized by adenine (A)/cytosine (C) base translocation at position 1166 of the gene. Different results have been obtained in studies attempting to explain the relationship between these gene polymorphisms and HCM development [12].

\subsubsection{CYP11B2 gene polymorphisms}

It has been determined that aldosterone, which can be produced locally in the heart, is associated with sarcomeric mutations and cardiac phenotype. The CYP11B2 -344C/T gene polymorphism is characterized by $\mathrm{C} / \mathrm{T}$ base displacement at the -344 position of the CYP11B2 gene localized on the $8 \mathrm{q} 22$ chromosome. The CYP11B2 gene polymorphism was found to be associated with left ventricular mass in human essential hypertension. In a previous study, aldosterone was found to modify the phenotypic expression of the mutated gene in HCM. A significant relationship between CYP11B2 genotype and cardiac hypertrophy has been shown in HCM. It has also been reported that the T allele of the CYP11B2 gene polymorphism in patients with essential hypertension has been identified as a genetic risk factor for left ventricular mass. In another study, a significant association was found between the CYP11B2 $-344 \mathrm{C} / \mathrm{T}$ gene polymorphism CC genotype and cardiac hypertrophy among healthy controls [14]. Several previous studies have reported that the T allele of the CYP11B2 gene polymorphism is associated with increased plasma aldosterone levels. Therefore, it is thought to be a significant relationship between $T$ allele of this gene polymorphism and cardiac hypertrophy [12].

\subsubsection{AGT gene polymorphisms}

AGT released into the circulation is a glycoprotein produced by hepatocytes containing 485 amino acids. It is known that AGT is converted into angiotensin I by the renin enzyme. The rate in angiotensin production plays a role in the regulation of AGT concentration and angiotensin II production. In addition, AGT plays an important role in essential hypertension, renal tubular dysgenesis, non-familial structural atrial fibrillation, and in LVH via strong myotrophic effect. The AGT gene is known to regulate the expression of AGT. AGT M235T gene polymorphism is characterized by methionine/threonine base displacement in chromosome 1q42 of the AGT gene [14]. In studies conducted to investigate the relationship between AGT M235T gene polymorphism and HCM, controversial results were found. Although in some studies significant relationships were determined, in some studies were not found. In a study conducted with the Japanese population by Kawaguchi et al., it was determined that TT genotype and T allele of the AGT M235T gene polymorphism were genetic risk factors for HCM. However, in the same study, no significant relationship was found between TT genotype and T allele of this gene polymorphism and familial form of HCM. A higher T allele frequency was found in patients with sporadic HCM. The TT genotype of the AGT M235T gene polymorphism is thought to be a genetic marker for LVH. It was also found to be significant relationship between this polymorphism and other cardiovascular diseases such as myocardial infarction, coronary atherosclerosis, and hypertension. In another study conducted with the South Indian population, the relationship between T704C gene polymorphism and HCM in exon 2 of the AGT gene was investigated. In this study, T allele of AGT T704C gene polymorphism was found to be associated with sporadic HCM. However, it was concluded that this allele is not a genetic risk factor for familial HCM. In conclusion, the T allele of the AGT 


\begin{tabular}{lll}
\hline SNPs & Forward primer $\left(5^{\prime}-\mathbf{3}^{\prime}\right)$ & Reverse primer $\left(5^{\prime}-\mathbf{3}^{\prime}\right)$ \\
\hline ACE (I/D) & 5'-CTGGAGAGCCACTCCCATCCTTTCT-3' & 5'-GACGTGGCCATCACATTCGTCAGAT-3' $^{\prime}$ \\
\hline AGTR1 & 5'-GAAGCCTGCACCATGTTTTGA-3' & 5'-GGCTTTGCTTTGTCTTGTTG-3' $^{\prime}$ \\
A1166C & & \\
\hline CYP11B2 & 5'-CAGGAGGAGACCCCATGTGAC-3' & 5'-CCTCCACCCTGTTCAGCCC-3' \\
-344C/T & & \\
\hline AGT & 5'-CAGGGTGCTGTCCACACTGGACCCC-3' & 5'-CCGTTTGTGCAGGGCCTGGCTCTCT-3' \\
M235T & & \\
\hline AGT & 5'-CAGGGTGCTGTCCACACTGGACCCC-3' & 5'-CCGTTTGTGCAGGGCCTGGCTCTCT-3' \\
T704C & & \\
\hline PCR, polymerase chain reaction; SNP, single nucleotide polymorphism.
\end{tabular}

Table 5.

Primer sequences used in PCR for RAAS gene polymorphisms.

T704C gene polymorphism has been reported to be associated with the development of sporadic HCM. A larger scale of cohort studies should be performed to confirm the relationship between T alleles and HCM development of these gene polymorphisms [3]. Primer sequences for RAAS gene polymorphisms are presented in Table $5[15,16]$.

\section{Conclusions}

It is known that genetic and environmental factors play a role in the pathogenesis of HCM. Numerous studies have been conducted to investigate gene polymorphisms playing the role in HCM development. The differences in the results of these studies are thought to be stemmed from different race and population characteristics and different selection criteria of patient and control groups in the study. The identification of genes and the polymorphisms occurring in these genes that are effective in the development of HCM will enable us to have knowledge about disease-related mechanisms in HCM susceptibility and to develop new drug and treatment strategies in the prevention of HCM. Different results can be obtained in studies with different and larger populations.

\section{Acknowledgements}

This chapter was performed by Nevra Alkanli and Arzu Ay from the Department of Biophysics in Halic University Medical Faculty and in Trakya University Medical Faculty.

\section{Conflict of interest}

We declare that there is no conflict of interest with any financial organization regarding the material discussed in the chapter. 
Genetic Polymorphisms that Playing Role in Development of Hypertrophic Cardiomyopathy DOI: http://dx.doi.org/10.5772/intechopen.83473

\section{Author details}

Nevra Alkanli ${ }^{1 *}$ and Arzu Ay ${ }^{2}$

1 Department of Biophysics, Faculty of Medicine, Halic University, Istanbul, Turkey

2 Department of Biophysics, Faculty of Medicine, Trakya University, Edirne, Turkey

*Address all correspondence to: nevraalkanli@halic.edu.tr

\section{IntechOpen}

(C) 2019 The Author(s). Licensee IntechOpen. This chapter is distributed under the terms of the Creative Commons Attribution License (http://creativecommons.org/licenses/ by/3.0), which permits unrestricted use, distribution, and reproduction in any medium, provided the original work is properly cited. (cc) BY 


\section{References}

[1] Tirone AP, Arteaga E. Familial hypertrophic cardiomyopathy. Genetic characterization. Arquivos Brasileiros de Cardiologia. 1999;72(4):520-522

[2] Iio C, Akiyoshi O, Nagai T, et al. Association between genetic variation in the SCN10A gene and cardiac conduction abnormalities in patients with hypertrophic cardiomyopathy. International Heart Journal.

2015;56:421-427

[3] Manohar Rao PPK, Anjana M, Mullapudi R, et al. The M235T polymorphism of the angiotensinogen gene in South Indian patients of hypertrophic cardiomyopathy. Journal of the Renin-Angiotensin-Aldosterone System. 2010;12(3):238-242. DOI: $10.1177 / 1470320310387955$

[4] Güleç Ç, Abacı N, Bayrak F. Association between non-coding polymorphisms of HOPX gene and syncope in hypertrophic cardiomyopathy. Anadolu Kardiyoloji Dergisi. 2014;14:617-624. DOI: 10.5152/ akd.2014.4972

[5] Ji F, Liu Q, Feng Z, et al.

Genetic association between 1425G/A SNP in PRKCH and hypertrophic cardiomyopathy in a Chinese population. Oncotarget. 2017;8(70):114839-114844

[6] Rodríguez R, Guerrero D, Rivas Y, et al. Genetic variations of $\beta-\mathrm{MYH} 7$ in Venezuelan patients with hypertrophic cardiomyopathy. Investigación Clínica. 2014;55(1):23-31

[7] Yuan Y, Meng L, Zhou Y, et al. Genetic polymorphism of angiotensinconverting enzyme and hypertrophic cardiomyopathy risk. A systematic review and meta-analysis. Medicine. 2017;96(e8639):48. DOI: $10.1097 /$ MD.0000000000008639
[8] Han K, Li Y-P. Prognostic predictive value of TLR4 polymorphisms in Han Chinese population with hypertrophic cardiomyopathy. Kaohsiung Journal of Medical Sciences. 2018;34:569-575

[9] Laredo R, Monserrat L, HermidaPrieto M. Beta-myosin heavy Chain gene mutations in patients with hypertrophic cardiomyopathy. Revista Española de Cardiología. 2006;59(10):1008-1018

[10] Kraker J, Viswanathan SK, Knöll R. Recent advances in the molecular genetics of familial hypertrophic cardiomyopathy in South Asian descendants. Frontiers in Physiology. 2016;7(499):1-14

[11] Rangaraju A, Satyanarayana ML, Ananthapur V, et al. Heat shock protein 70 polymorphism in hypertrophic cardiomyopathy of South Indian cohort. Journal of Indian College of Cardiology. 2013;3:9-15. DOI: 10.1016/j. jicc.2012.12.007

[12] Chai W, Hoedemaekers Y, van Schaik RH, et al. Cardiac aldosterone in subjects with hypertrophic cardiomyopathy. Journal of the ReninAngiotensin-Aldosterone System. 2006;7(4):225-230. DOI: 10.3317/ jraas. 2006.042

[13] Kaufman BD, Auerbach S, Reddy S. RAAS gene polymorphisms influence progression of pediatric hypertrophic cardiomyopathy. Human Genetics. 2007;122:515-523. DOI: 10.1007/ s00439-007-0429-9

[14] Ortlepp JR, Vosberg HP, Reith S. Genetic polymorphisms in the renin-angiotensin-aldosterone system associated with expression of left ventricular hypertrophy in hypertrophic cardiomyopathy: a study of five polymorphic genes in a family with a disease causing mutation in the 
Genetic Polymorphisms that Playing Role in Development of Hypertrophic Cardiomyopathy DOI: http://dx.doi.org/10.5772/intechopen.83473

myosin binding protein $\mathrm{C}$ gene. Heart.

2002;87:270-275

[15] Ramu P, Umamaheswaran G, Shewade DG, et al. Candidate gene polymorphisms of renin angiotensin system and essential hypertension in a South Indian Tamilian population. International Journal of Human Genetics. 2011;11(1):31-40

[16] Kaplan İ, Sancaktar E, Ece A. Gene polymorphisms of adducin GLY460TRP, ACE I/D, and AGT M235T in pediatric hypertension patients. Medical Science Monitor. 2014;20:1745-1750. DOI:

10.12659/MSM.892140 


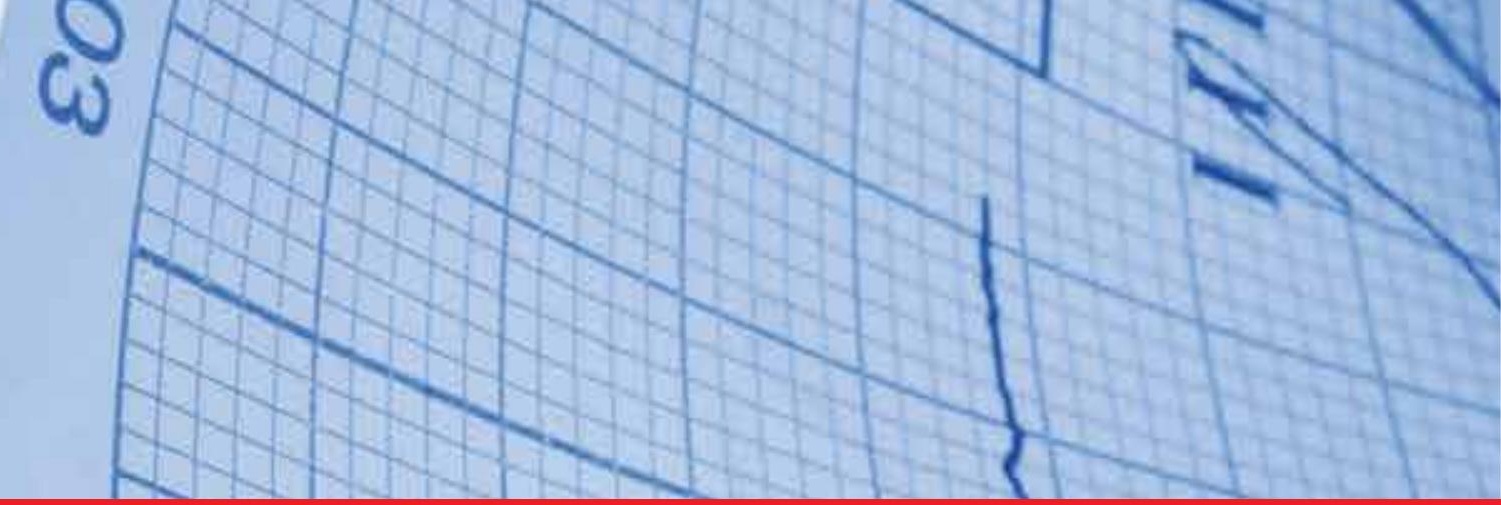

\section{Edited by Umashankar Lakshmanadoss}

This book provides an excellent overview of the diagnosis of abnormal electrocardiograms (ECGs) through deep learning methods. These methods include optimal techniques that can link the processing and analysis of nonstationary ECG signals, the various statistical methods of converting ECG data into variant maps, and the application of various ways of identifying premature atrial beats, ECG characteristics of right and left ventricular tachyarrhythmia, and conditions producing left ventricular hypertrophy, including hypertrophic cardiomyopathy. This book is divided into two sections, including basic and practical applications of ECGs. We hope that it will serve as a reference for the techniques used to obtain and process electrical signals for ECGs. This book will also function as an excellent reference for atrial and ventricular tachyarrhythmia.

\section{IntechOpen}
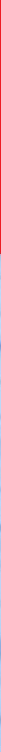